\title{
Early Jurassic Ammonites from Alaska
}

GEOLOGICAL SURVEY PROFESSIONALAAPER 1148

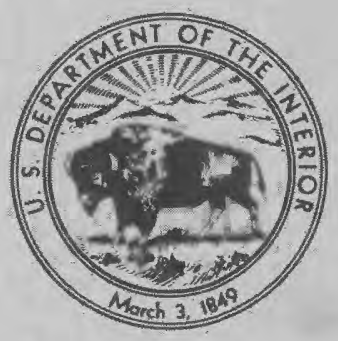




\section{Early Jurassic Ammonites from Alaska}

By RALPH W. IMLAY

GE OLOGICAL S UR VEY PROFESSIONALA A PER 1148

Studies of Early Jurassic ammonites from Alaska provide close correlations with Lower Jurassic ammonite zones in Europe and with ammonite successions elsewhere in the world

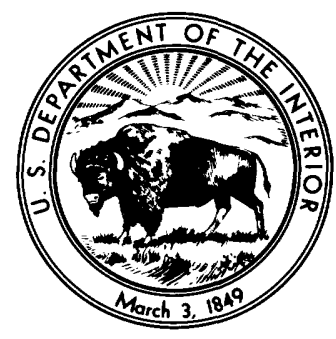


UNITED STATES DEPARTMENT OF THE INTERIOR

JAMES G. WATT, Secretary

GEOLOGICAL SURVEY

Doyle G. Frederick, Acting Dircetor

\section{Library of Congress Cataloging in Publication Data}

Imlay, Ralph Willard, 1908-

Early Jurassic ammonites from Alaska.

(Geological Survey professional paper ; 1148)

Bibliography: $p$.

Supt. of Docs. no.: I 19.16:1148

Includes index.

1. Ammonoidea. 2. Paleontology-Jurassic.

QE807.A51585 564'.53 81-607901

3. Paleontology-Alaska. I. Title. II. Series.

AACR2

For sale by the Superintendent of Documents, U.S. Government Printing Office Washington, D.C. 20402. 


\section{CONTENTS}

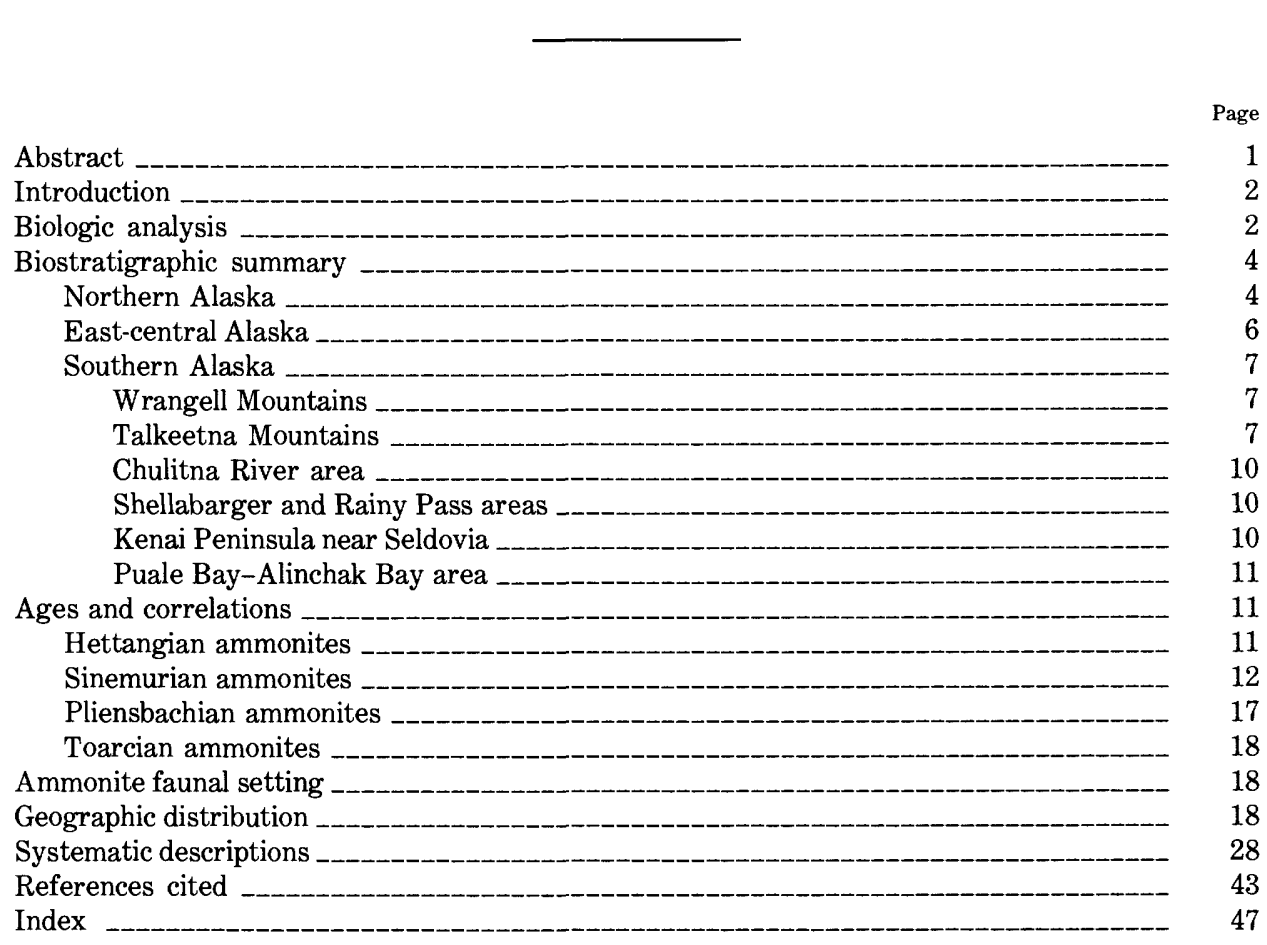

\section{ILLUSTRATIONS}

[Plates 1-12 follow index]

Plate 1. Psiloceras, P. (Franziceras), and Discamphiceras.

2. Waehneroceras, Schlotheimia, and Badouxia.

3. Charmasseiceras, Paltechioceras, and Laqueoceras.

4. Coroniceras, C. (Paracoroniceras), Arietites, and Paltechioceras.

5. Arctoasteroceras, Amioceras, and Crucilobiceras.

6. Paracaloceras.

7. Crucilobiceras and Fanninoceras.

8. Tropidoceras, Crucilobiceras, Uptonia?, and Apoderoceras.

9. Uptonia.

10. Arieticeras, Prodactylioceras, Arieticeras, and Amaltheus.

11. Pseudolioceras, Dactylioceras, D. (Orthodactylites), Grammoceras, Eleganticeras, Harpoceras, Leptaleoceras, Protógrammoceras, and Fontanelliceras.

12. Haugia, Catacoeloceras?, Brodieia, Protogrammoceras, Pseudolioceras, and Phymatoceras?.

Figure 1. Generalized index map of Lower Jurassic ammonite localities in Alaska

2-6. Detailed index maps of:

2. Lower Jurassic ammonite localities in the Wrangell Mountains, southern Alaska

3. Lower Jurassic ammonite localities in the Nelchina area of the Talkeetna Mountains and in the upper part of the Matanuska Valley, southern Alaska

4. Lower Jurassic ammonite localities in the Healy (A-6) quadrangle, Chulitna River area, southern Alaska _._.........

5. Lower Jurassic ammonite localities in the Seldovia area on the Kenai Peninsula, southern Alaska

6. Lower Jurassic ammonite localities in the Puale Bay-Alinchak Bay area, Alaska Peninsula 
FIGURE 7. Diagram of European ranges of Early Jurassic ammonite genera present in Alaska

8. Diagram correlating Early Jurassic ammonite faunas in northern and east-central Alaska

9. Diagram correlating Early Jurassic ammonite faunas in southern Alaska

10-12. Diagrams showing occurrences and ages of:

10. Ammonites present in the Lower Jurassic beds exposed in the Wrangell Mountains in southern Alaska

11. Early Jurassic ammonites present in the Nelchina area of the Talkeetna Mountains and in the upper part of the Matanuska River Valley, southern Alaska

12. Ammonites present in unnamed Lower Jurassic beds on northeast side of Puale Bay on the Alaska Peninsula

13. Diagram correlating Lower Jurassic rocks in northern and east-central Alaska

14. Diagram correlating Lower Jurassic rocks in southern Alaska

\section{TABLES}

TABLE 1. Ammonite genera and subgenera of Early Jurassic age in Alaska

2. Early Jurassic ammonites from well cores in northern Alaska

3-6. Geographic distribution of:

3. Early Jurassic ammonites from outcrops in northern and east-central Alaska

4. Early Jurassic ammonites in the Yakutat area and in the Wrangell Mountains in southern Alaska

5. Early Jurassic ammonites in the Talkeetna Mountains in southern Alaska

6. Early Jurassic ammonites in the Chulitna River area of the Alaska Range, in the Seldovia area of the Kenai Peninsula, and in the Puale Bay-Alinchak Bay and Wide Bay areas of the Alaska Peninsula 


\title{
EARLY JURASSIC AMMONITES FROM ALASKA
}

\author{
By RALPH W. IMLAY
}

\begin{abstract}
Early Jurassic ammonites of early Hettangian to late Toarcian Age are present in the lower part of the Kingak Shale and equivalent beds in northern Alaska. Well cores from the Arctic Coastal Plain contain ammonites of early Hettangian to Sinemurian Age and of late Pliensbachian to early Toarcian Age. Surface exposures have furnished ammonites of early Pliensbachian to late Toarcian Age. In addition the presence of the pelecypod Otapiria tailleuri Imlay near the base of the Lower Jurassic exposures throughout much of northern Alaska is good evidence of a Sinemurian Age because this pelecypod occurs in the subsurface with ammonites of early Sinemurian Age and it occurs on the surface near, but not on the same slabs as, Uptonia of earliest
\end{abstract} Pliensbachian Age.

Ammonites of early Hettangian and late Pliensbachian Age are present in part of the Glenn Shale exposed near Old Rampart in eastcentral Alaska.

In the Wrangell Mountains (lat $62^{\circ} \mathrm{N}$., long $141^{\circ}-143^{\circ} \mathrm{W}$.), the upper member of the McCarthy Formation has furnished ammonites of early Hettangian to early Sinemurian Age and of latest Sinemurian to fairly early Pliensbachian Ages. The overlying Lubbe Creek Formation contains ammonites of middle to late Pliensbachian Age and possibly also of early Toarcian Age.

In the Talkeetna Mountains, northeast of Anchorage, the exposed upper part of the Talkeetna Formation has furnished ammonites of latest Sinemurian to earliest Pliensbachian Age and of late Pliensbachian to late Toarcian Age.

In the Chulitna River area, only ammonites of early and latest Sinemurian Age have been found. In the Seldovia area, only ammonites of earliest Hettangian and early Sinemurian Age have been found. In the Puale Bay area, the Hettangian and lower Sinemurian are well represented by ammonites in an unnamed formation. Sharply above lies the Kialagvik Formation, which is mostly of Bajocian Age, but which contains the ammonite Haugia of middle to early late Toarcian Age about $30 \mathrm{ft}(9 \mathrm{~m})$ above its base.

These data show that parts of all these Lower Jurassic sequences are not represented by ammonites or are poorly represented. Thus ammonites of late Hettangian and middle Pliensbachian Age are represented only by single occurrences, and the Sinemurian zones of Caenisites turneri to Oxynoticeras oxynotum are not represented by any ammonite occurrences.

The Hettangian ammonite succession in Alaska is similar to that in western Europe and probably includes species in common. Its lower part, equivalent to the Psiloceras planorbis zone, is represented in many areas by $P$. cf. $P$. planorbis (J. de C. Sowerby), or by P. (Franziceras) cf. $P$. (F.) ruidum (Buckman), or by both. Its middle part, probably equivalent to the Alsatites liasicus zone, is represented in the Wrangell Mountains by Discamphiceras cf. D. toxophorum (Gümbel), at Wide Bay (lat $57^{\circ} 45^{\prime}$ N., long $156^{\circ}$ W.) by Waehneroceras cf. $W$. portlocki (Wright), and at the Puale Bay-Alinchak Bay area by these taxa plus $W$. cf. $W$. tenerum (Neumayr) and Laqueoceras cf. $L$. sublaqueus (Waehner). The upper Hettangian is represented by a single specimen of Schlotheimia in the Puale Bay area.
The Sinemurian ammonite succession in Alaska is likewise similar to that in western Europe but does not include genera that are definitive for nearly half of the stage (Caenisites turneri-Oxynoticeras oxynotum zones). Its lowest part, equivalent to the Arietites bucklandi zone of Europe, is represented in the subsurface of the Arctic Coastal Plain by Arietites cf. A. bucklandi (Sowerby), by Charmasseiceras, and by species of Coroniceras both below and above Charmasseiceras. Its lowest part in southern Alaska is represented in the Chulitna River, Seldovia, and Puale Bay areas by Paracaloceras rursicostatum Frebold, which in the Chulitna area is associated with Badouxia canadensis (Frebold).

The overlying beds in most of these areas are characterized by $A r$ nioceras, whose presence and stratigraphic position are evidence for correlation with the late early Sinemurian Arnioceras semicostatum zone of Europe. Such an age for Arnioceras in the Arctic Coastal Plain is confirmed by its position below that of Coroniceras, a genus not known above the $A$. semicostatum zone. Such an age for Coroniceras (Paracoroniceras) in the Seldovia area is shown by the known range of the subgenus in Europe.

The highest part of the Sinemurian, representing the Echioceras raricostatum zone, has been identified by the occurrence of Paltechioceras in the Wrangell and Talkeetna Mountains and in the Chulitna River area. That genus in the Talkeetna Mountains is associated with Crucilobiceras, which ranges upward into the lower Pliensbachian. In the Chulitna River area Paltechioceras is associated with Arctoasteroceras, a genus that in arctic Canada occurs with $O x$ ynoticeras oxynotum (Quenstedt).

The Pliensbachian ammonite succession in northern Alaska is incomplete. At its base, the Uptonia jamesoni zone is represented by Uptonia cf. $U$. jamesoni (J. de C. Sowerby) obtained from outcrops near the Ipnavik and Etivluk Rivers (tributaries of the Colville River). The next two higher zones of western Europe have not been recognized. The still higher zone of Amaltheus margaritatus is present throughout northern Alaska as well as farther southeast in the Old Rampart area of east-central Alaska. The highest zone of Pleuroceras spinatum is represented by Amaltheus (Pseudoamaltheus) engelhardti in the subsurface of the Arctic Coastal Plain. These occurrences of Amaltheus in abundance at many places furnish exact correlations with the upper Pliensbachian beds of northern Eurasia.

Pliensbachian ammonite successions in southern Alaska have been identified only in the Wrangell and Talkeetna Mountains and questionably in the Yakutat district. The Uptonia jamesoni zone is represented in the Wrangell Mountains by Uptonia ef. $U$. dayiceratoides Mouterde, in the Talkeetna Mountains by A poderoceras, and probably in the Yakutat district by a fragment that resembles the outer whorl of Uptonia rather than Crucilobiceras. The next higher zone of Tragophylloceras ibex, is represented by Tropidoceras actaeon (d'Orbigny) in association with Crucilobiceras. The Prodactylioceras davoei zone is possibly represented in the Wrangell Mountains by some float specimens of Prodactylioceras that were collected along with Uptonia. The highest two zones of the Pliensbachian are represented by the ammonites Protogrammoceras, Leptaleoceras, Arieticeras, and Fontannelliceras, which are identical 
or nearly identical with species in the Mediterranean area. With these taxa at one locality in the Talkeetna Mountains were found two specimens of Amaltheus.

This association with Amaltheus furnishes a correlation with the upper Pliensbachian of northern Alaska. Otherwise the late Pliensbachian ammonites in southern Alaska have nothing in common generically with ammonites of that age in the Arctic region. The same genera do occur farther south, however, in western British Columbia and in eastern Oregon, as well as in the Mediterranean area.

The Toarcian ammonite succession in Alaska is similar to that in western Europe and probably includes species in common. Characteristic taxa from the base upward include (1) Dactylioceras and Catacoeloceras (?), (2) Harpoceras cf. H. exaratum (Young and Bird) and Eleganticeras, (3) Haugia, Brodieia, and Pseudolioceras, and (4) Pseudolioceras and Grammoceras. The most complete Toarcian ammonite sequence is in the Talkeetna Mountains.

\section{INTRODUCTION}

Early Jurassic ammonites obtained from eight areas in northern, eastern, and southern Alaska (figs. 1-6) are described herein in order to present all available evidence concerning the stratigraphic and geographic distribution of the taxa (figs. 7-9), to evaluate their faunal setting in relation to other parts of the world, to make regional and continental correlations, and to date the formations in Alaska as precisely as possible in terms of the standard Jurassic zones of western Europe (figs. 10-14).

This study is based mainly on fossil collections and stratigraphic data furnished by many U.S. Geological Survey geologists, as listed in Imlay and Detterman $(1973$, p. 8,9$)$. It is also based on excellent fossil collections and data obtained by British Petroleum (Alaska), Inc., in 1964 in northern Alaska and by the Richfield Oil Co. (now Atlantic Richfield Oil Co.) in 1962 on the east shore of Puale Bay in the Alaska Peninsula. Biostratigraphic data for northern Alaska were furnished by W. P. Brosgé, R. L. Detterman, and H. N. Reiser; for east-central Alaska, by E. M. MacKevett, Jr.,; for the Talkeetna Mountains, by Arthur Grantz; and for the Chulitna Valley, by D. L. Jones.

The described specimens are deposited in the type collections of the U.S. National Museum and are labeled USNM.

\section{BIOLOGIC ANALYSIS}

Alaska ammonites of Early Jurassic (Hettangian to Toarcian) age described or mentioned herein number about 500 specimens whose distribution by family, subfamily, genus, and subgenus is shown on table 1 . This table shows that the families present include the Arietitidae (15 percent), Polymorphitidae (20 percent), Schlotheimiidae (16 percent), Hildoceratidae (15 percent), Psiloceratidae (9 percent), Hammoceratidae (6 percent), and Eoderoceratidae ( 6 percent). The remaining families combined represent only 13 percent of the total number of specimens.
The classification shown in table 1 is essentially that proposed by Donovan and Forsey (1973 p. 2-4), except for Fanninoceras and Arctoasteroceras, which those writers consider to be synonyms of Radstockiceras and Aegasteroceras, respectively. Nonetheless, Fanninoceras (McLearn, 1930, p. 4, 5; 1932, p. 76-80) differs morphologically from Radstockiceras by having a thinner whorl section and an undercut umbilical wall (Frebold, 1967a, p. 1146). It differs timewise by occurring in beds of late Pliensbachian Age in eastern Oregon (Imlay, 1968, p. C10, C11) and in beds of earliest to latest Pliensbachian Age in the Queen Charlotte Islands (Hans Frebold, written commun., 1975), whereas Radstockiceras in northwest Europe is characteristic of

TABLE 1.-Ammonite genera and subgenera of Early Jurassic age in Alaska

\begin{tabular}{|c|c|c|}
\hline Family & Genus and subgenus & $\begin{array}{l}\text { Number of } \\
\text { specimens }\end{array}$ \\
\hline Lytoceratidae ___-_ & ______L_Lytoceras ________ & 1 \\
\hline \multirow[t]{4}{*}{ Psiloceratidae _-_-_ } & _-_-_Psiloceras______-_ & 10 \\
\hline & (Franziceras) & 14 \\
\hline & Discamphiceras__- & 19 \\
\hline & Laqueoceras____-_ & 1 \\
\hline \multirow{4}{*}{ Schlotheimiidae _-- } & _Schlotheimia____- & 4 \\
\hline & Waehneroceras _.. & 63 \\
\hline & Badouxia_______ & 4 \\
\hline & Charmasseiceras _._ & 8 \\
\hline \multirow[t]{7}{*}{ Arietitidae _____- } & Arietitinae _______Arietites________ & 1 \\
\hline & Coroniceras & 17 \\
\hline & (Paracoroniceras) & 3 \\
\hline & Arnioceras______ & 45 \\
\hline & Arnioceras?______ & 1 \\
\hline & Paracaloceras _-_- & 10 \\
\hline & Asteroceratinae___Arctoasteroceras _-_ & 2 \\
\hline \multirow[t]{2}{*}{ Echioceratidae__-_- } & _-___________echioceras____ & 10 \\
\hline & (Orthechioceras)_- & 1 \\
\hline \multirow{3}{*}{ Eoderoceratidae -- } & Xipheroceratinae__Crucilobiceras____ & 26 \\
\hline & Coeloceratinae___Apoderoceras__-_-- & 2 \\
\hline & Apoderoceras? & 1 \\
\hline Oxynoticeratidae__- & 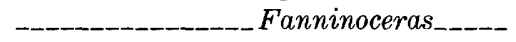 & 16 \\
\hline \multirow[t]{3}{*}{ Polymorphitidae _- } & - Uptonia ____-_ & 81 \\
\hline & Uptonia? & 9 \\
\hline & Tropidoceras _-_-_- & 4 \\
\hline \multirow[t]{2}{*}{ Amaltheidae____-_ } & $\begin{array}{l}\text { Amaltheus } \\
\quad \text { (Pseudo- }\end{array}$ & 18 \\
\hline & amaltheus) & 1 \\
\hline \multirow[t]{4}{*}{ Dactylioceratidae } & ___odactylioceras _- & 4 \\
\hline & Dactylioceras & 6 \\
\hline & (Orthodactylites)_ & 7 \\
\hline & Catacoeloceras? _-- & 1 \\
\hline \multirow[t]{8}{*}{ Hildoceratidae _-_- } & Arieticeratinae____Arieticeras_____-_ & 42 \\
\hline & Leptaleoceras__-_-- & 1 \\
\hline & Fontanelliceras__-_ & 5 \\
\hline & Harpoceratinae ___Harpoceras _____- & 5 \\
\hline & Protogrammoceras & 5 \\
\hline & Elegantuliceras _-_ & 12 \\
\hline & Pseudolioceras & 5 \\
\hline & Grammoceratinae _ Grammoceras? ---- & 1 \\
\hline \multirow[t]{3}{*}{ Hammatoceratidae } & Phymatoceratinae_Phymatoceras?____- & 3 \\
\hline & Brodieia & 1. \\
\hline & Haugia & 28 \\
\hline
\end{tabular}




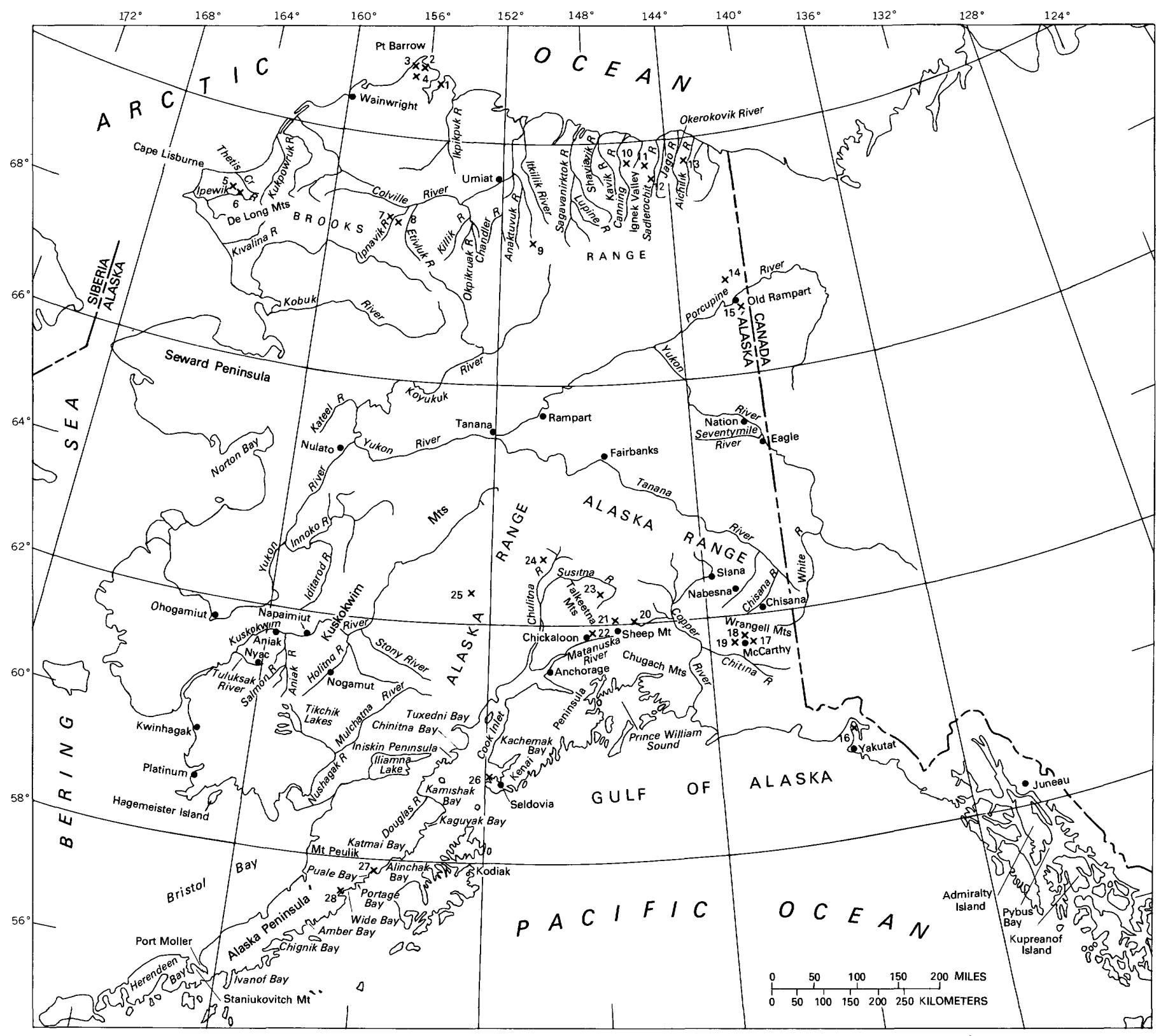

FiguRE 1.-Generalized index map of Lower Jurassic ammonite areas in Alaska, as listed in tables 3-7.

the earliest Pliensbachian (Dean and others, 1961, p. 463, fig. 7).

The genus Arctoasteroceras of Frebold (1960, p. 13, 14, pl. 2 figs. 1-5, pl. 3, figs. 1-3) differs morphologically from Aegasteroceras of Spath (1925, p. 265, 267, fig. 6a) by having weak ribs on the upper parts of the flanks and on the venter and commonly by having an asymmetrical suture line. These differences were not accepted as valid by Hallam $(1965$, p. 1495) or by Donovan and Forsey $(1973$, p. 3). Nonetheless, some European specimens of Aegasteroceras, including the holotype of the genotype species, A. simile Spath (Guerin-Franiatte, 1966, p. 310-313, pls. 189-192), bear strong ribs on the upper part of the flanks and on the margins of the venter, and some ribs are weakly connected across the venter. This strong ribbing contrasts markedly with the weak ribs on Arctoasteroceras jeletzkyi Frebold from Canada.

These genera likewise differ slightly in age. Thus, Aegasteroceras in Europe occurs in the zone of Asteroceras obtusum of earliest late Sinemurian Age (Dain and others, 1961, p. 454). Arctoasteroceras jeletzkyi in Canada is dated as middle late Sinemurian because of its occurrence with Oxynoticeras oxynotum and below echioceratid ammonites (Frebold, 1960, p. 14, 26). The Alaskan specimens of Arctoasteroceras described herein are dated as probably latest Sinemurian 


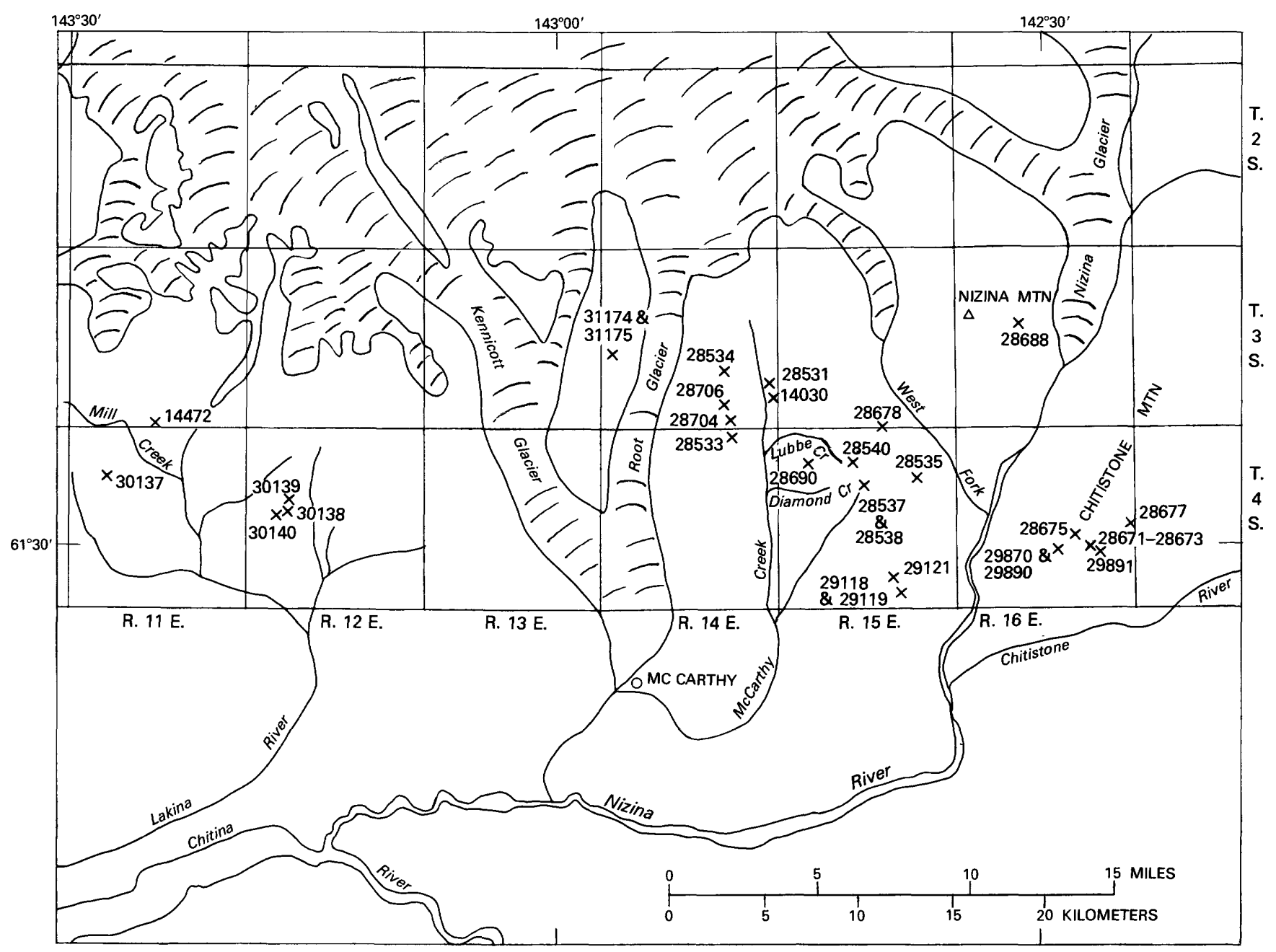

FIGURE 2.-Detailed index map of Lower Jurassic ammonite localities in the Wrangell Mountains, southern Alaska.

because they were collected with Paltechioceras (Orthechioceras?), which in Europe occurs only in the Echioceras raricostatum zone (Getty, 1973, p. 6).

The characteristics of the Early Jurassic ammonites described herein agree very well with those of various genera and subgenera defined in the "Treatise on Invertebrate Paleontology" (Arkell and others, 1957). In addition, the taxonomy of the Psiloceratidae has been discussed by Donovan (1952, p. 634, 641) and Frebold (1967b, p. 17); the Schlotheimiidae by Lange (1951, p. 23, 25), Donovan (1952, p. 644-655), Frebold (1960, p. 13), and Guex and Taylor (1976, p. 525); the Arietitidae by Donovan $(1952$, p. $717,725,739,746)$, Donovan and Forsey $(1973$, p. 6, 7), and Guerin-Franiatte $(1966$, p. $106-118,150,252,281,283,309,310,313)$; the Echioceratidae by Getty $(1973$, p. $7,8,17-21,23,24)$; the Amaltheidae by Howarth (1958, pt. 1, p. 1, 21; pt. 2); the Polymorphitidae by Bremer (1965, p. 177); the Dactylioceratidae by Pinna and Levi-Setti (1971) and
Schmidt-Effing (1972); the Polymorphitidae, Dactylioceratidae, and Hildoceratidae by Geczy (1976); and the Dactylioceratidae by Fischer (1966, p. 20-48). In addition, the taxonomy of the Early Jurassic ammonite families of southern Germany has been briefly described by Schlegelmilch (1976, p. 32-95).

\section{BIOSTRATIGRAPHIC SUMMARY NORTHERN ALASKA}

Lower Jurassic marine beds cropping out in northern Alaska (figs. 1, 13) have been briefly described or mentioned by Imlay and Detterman (1973, p. 9; p. 12 fig. 11B). Those from the Sagavanirktok River eastward have been described in considerable detail by Detterman and others $(1975$, p. 18-20). In general the outcrops are poorly exposed and sparsely fossiliferous, but they have furnished fossils representing most of the Pliensbachian and Toarcian Stages and perhaps the Sinemurian (fig. 8). Field studies to 1978 are insufficient to demonstrate 


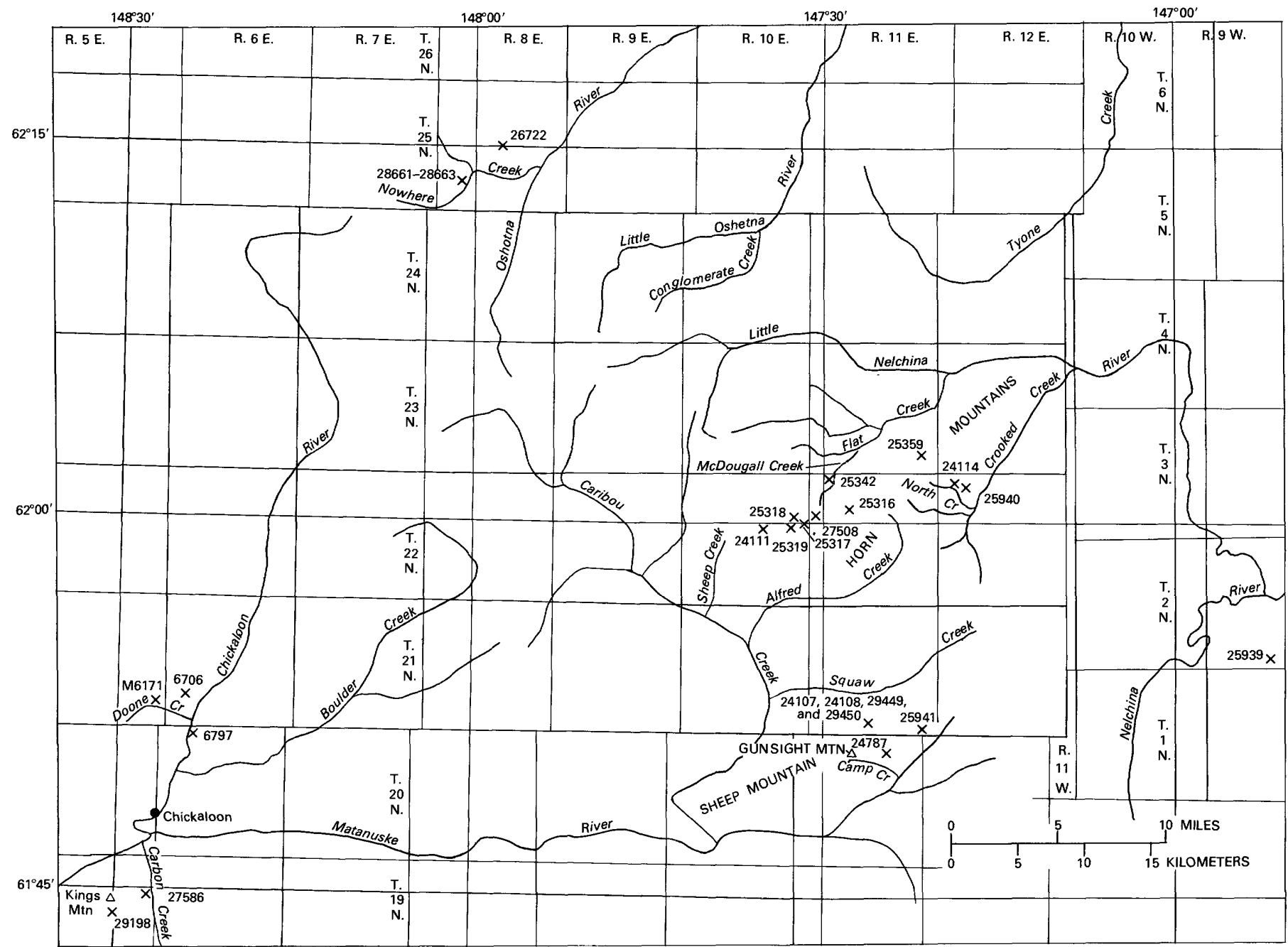

Figure 3.-Detailed index map of Lower Jurassic ammonite localities in the Nelchina area of the Talkeetna Mountains and in the upper part of the Matanuska Valley, southern Alaska.

whether the absence of earlier Jurassic is due to lack of deposition, poor outcrops, or insufficient field work in poorly fossiliferous beds.

The westernmost exposures in the DeLong Mountains consist mostly of clay shales that bear limestone concretions, are of unknown thickness, and have furnished ammonites of late Pliensbachian to early Toarcian Age. Next to the east between the Ipnavik and Itkillik Rivers, the Lower Jurassic apparently consists of 60 feet $(18 \mathrm{~m})$ or less of siliceous claystone that locally contains some limestone and is dated as Sinemurian to early Pliensbachian. Such an age is shown by the widespead distribution of Otapiria tailleuri Imlay, which in the subsurface is found associated only with ammonites of early Sinemurian Age, and by the presence on the surface of the early Pliensbachian ammonite Uptonia in slabs that do not contain Otapiria, although that genus occurs nearby.

Still farther east, in the area between the
Sagavanirktok and Aichilik Rivers, the Lower Jurassic beds (Detterman and others, 1975, p. 18-20, 44) represent the lower part of the Kingak Shale and range in thickness from 100 feet $(30 \mathrm{~m})$ or less, to about 900 feet $274 \mathrm{~m}$. This lower part consists of fissile black papery shale that at the Aichilik River is about 600 feet $(180 \mathrm{~m})$ thick and on the Kavik River contains Otapiria tailleuri Imlay of Sinemurian Age. The upper part consists of dark-gray clay shale and claystone that in places is as much as 300 feet (92 m) thick and contains ammonites of late Pliensbachian to late Toarcian Age.

The Lower Jurassic sequences in the subsurface of northern Alaska differ from those on the surface mainly by containing minor amounts of glauconitic sandstone, by being somewhat more fossiliferous, and by containing ammonites representing all Lower Jurassic stages (table 2). They have not, however, furnished any ammonites representing middle Sinemurian to middle Pliensbachian time, or late Toarcian time. 


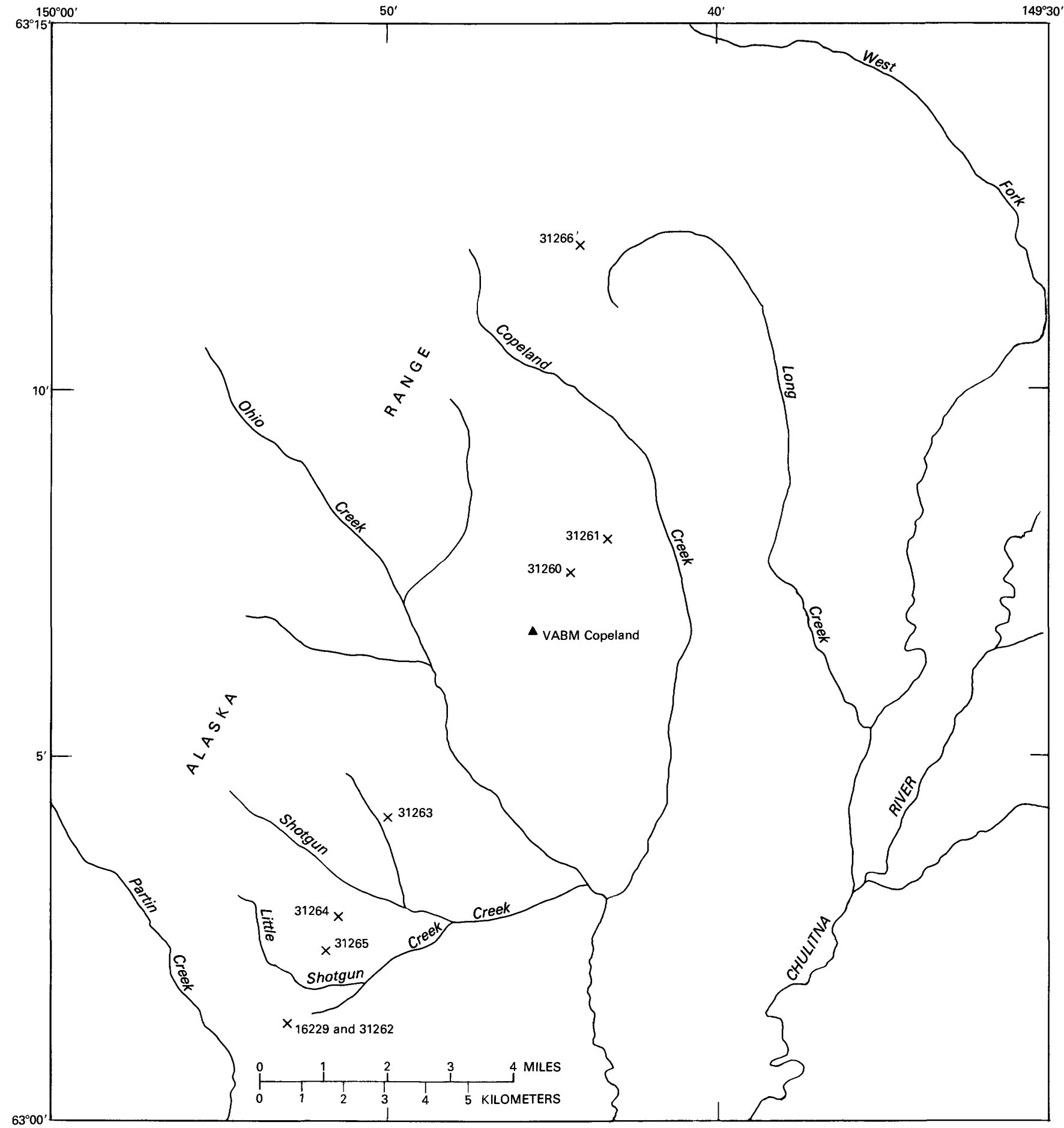

FiguRe 4.-Detailed index map of Lower Jurassic ammonite localities in the Healy (A-6) quadrangle, Chulitna River area, southern Alaska.

EAST-CENTRAL ALASKA

In the northern part of east-central Alaska, the Lower Jurassic is poorly exposed but is reported to consist of about 1000 feet $(305 \mathrm{~m})$ of sandstone, siltstone, shale, and quartzite that are part of the Glenn Shale (Brosgé and Reiser 1964, 1969; Imlay and Detterman, 1973, p. $13,14)$. The basal 120 feet $(37 \mathrm{~m})$ exposed on Spike Mountain about 30 miles $(48 \mathrm{~km})$ north of Old Rampart, consists of sandy beds, rests on granite, and contains the Hettangian ammonites Psiloceras (Psiloceras) and 


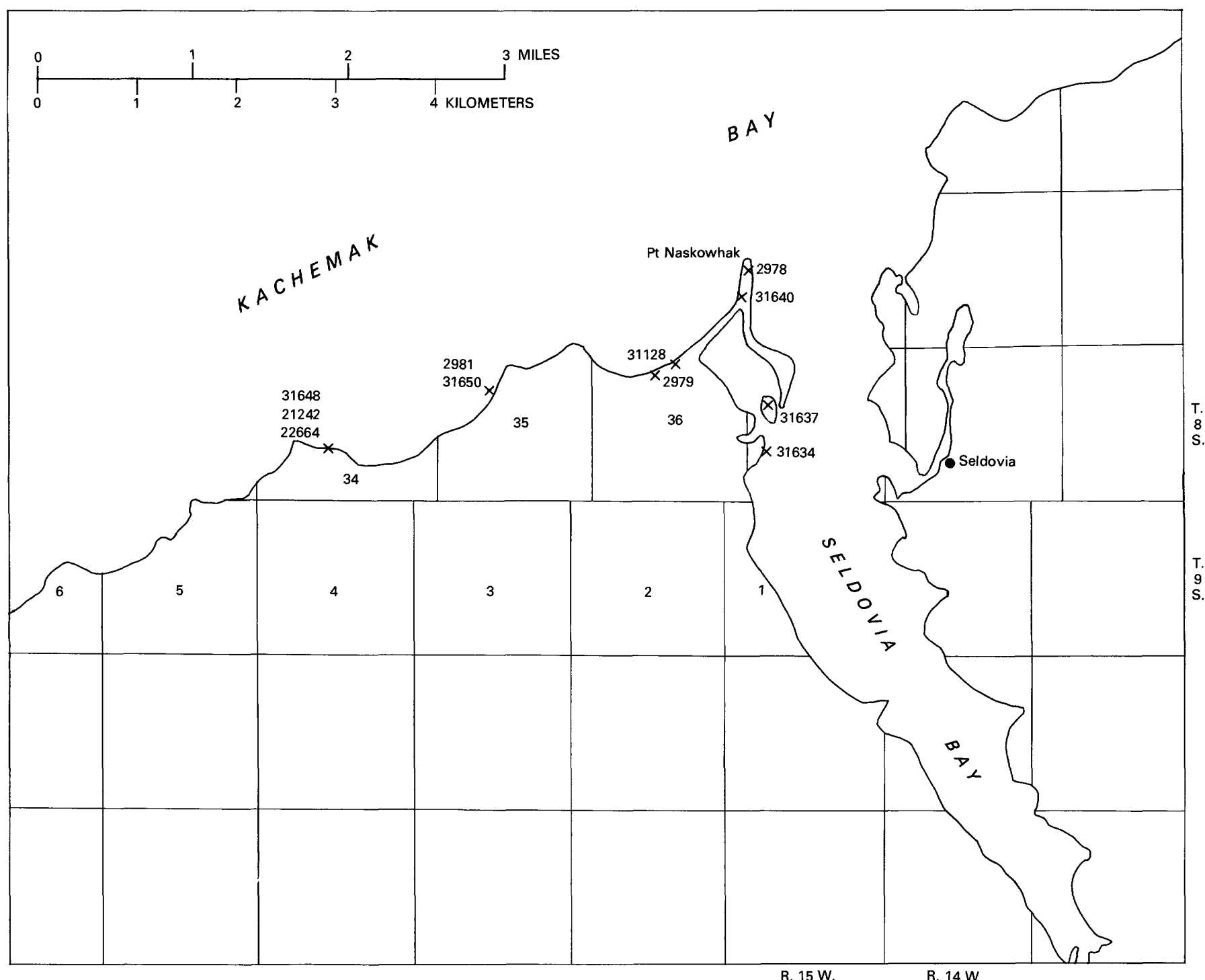

FIgURE 5.-Detailed index map of Lower Jurassic ammonite localities in the Seldovia area (Seldovia (B-5) quadrangle) on the Kenai Peninsula, southern Alaska.

P. (Franziceras) throughout. The only other Jurassic ammonite from the same general area is a specimen of Amaltheus stokesi (J. Sowerby) obtained about 9 miles $(14.4 \mathrm{~km})$ east-southeast of Old Rampart.

\section{SOUTHERN ALASKA}

WRANGELL MOUNTAINS

The Lower Jurassic sequence in the Wrangell Mountains has been described in detail by MacKevett (1969, $1970,1971)$; has been mentioned briefly by Imlay and Detterman $(1973$, p. 8,11$)$ regarding its position relative to a Jurassic volcanic island-arc system; and is depicted herein (figs. 10,14) in regard to fossil occurrences and ages. Most of the sequence is represented by the upper member of the McCarthy Formation which consists of
2,000-2,500 feet (610-760 m) of very thin to mediumbedded, dark-gray laminated chert and spiculite, grades into adjoining lithologic units, and is of Hettangian, Sinemurian, and earliest Pliensbachian Age. At the top, of the sequence is the Lubbe Creek Formation, which consists of 100-300 feet (30-91 m) of medium-gray spiculite and chert, is overlain unconformably by beds of Bajocian Age, and is of middle and late Pliensbachian to possibly early Toarcian Age.

\section{TALKEETNA MOUNTAINS}

The Lower Jurassic sequences in the Nelchina area of the Talkeetna Mountains and in the adjoining upper part of the Matanuska River valley (figs. 11, 14) have been studied by Arthur Grantz. Most of the lithologic and 


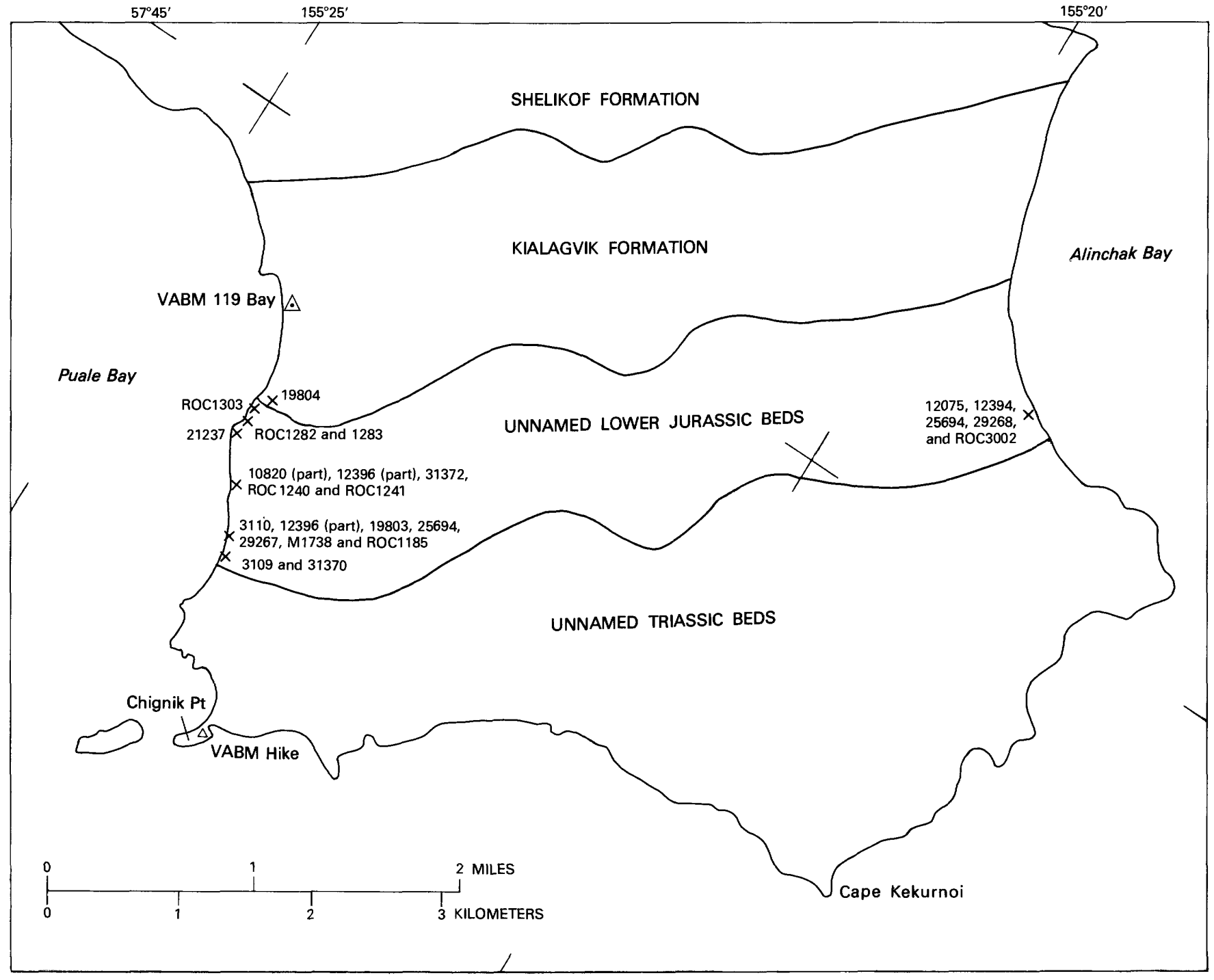

FIgURe 6.-Detailed index map of Lower Jurassic ammonite localities in the Puale Bay-Alinchak Bay area, Alaska Peninsula.

stratigraphic data shown in figure 11 were prepared by Grantz (written commun., 1977), along with these comments:

The Talkeetna Formation comprises a diverse assemblage of volcanic arc deposits. Waterlain pyroclastic and tuffaceous sedimentary rocks are much dominant over lava flows. If all rocks mapped as Talkeetna Formation are correctly assigned, its composition ranges from basaltic to rhyolitic, with andesitic rocks apparently dominant. The Talkeetna volcanic breccias and tuffs were commonly, and perhaps dominantly, deposited in marine waters. Marine fossils are widespread, and locally abundant in the waterlaid tuffs and in associated more-or-less tuffaceous sands and lutites. However, the presence of thick intervals of coarse volcanic breccias, tuffs and some flows that are barren of fossils and some outcrops that are rich in plant fossils suggest that nonmarine facies may also constitute an important part of the formation. Presumably, the fossiliferous marine beds represent inter-volcanic vent or arc-distal facies, and the pyroclastic and plant-rich beds represent submarine and subaerial composite volcanic cones that formed at major volcanic vents.
The Talkeetna Formation in the upper Matanuska Valley and Nelchina area occurs in large but well-separated outcrop areas. The lack of mapping continuity combined with moderate to locally great structural complexity, locally intense plutonism, and some metamorphism makes the recognition of the stratigraphic succession in these rocks difficult. The chief problem is the possibility, indeed probability, that the local lithologic succession in each isolated outcrop area of the formation owes as much, or more, to its geographic position (i.e., facies) as to its biostratigraphic position. However, if the prior assumption is made that the local succesions do have regional validity, then [a] $*^{* *}$ gross, and incomplete, succession can be inferred. Even with this assumption, however, the reconstruction given does not represent the only possible explanation of the available facts.

On the basis of these data and inferences, the Talkeetna Formation of the Talkeetna Mountains is $15,000-19,000$ feet $(4,660-5,790 \mathrm{~m})$ thick, is incompletely exposed basally, is dominated by volcanic rocks, is mostly marine, and contains ammonites of latest 


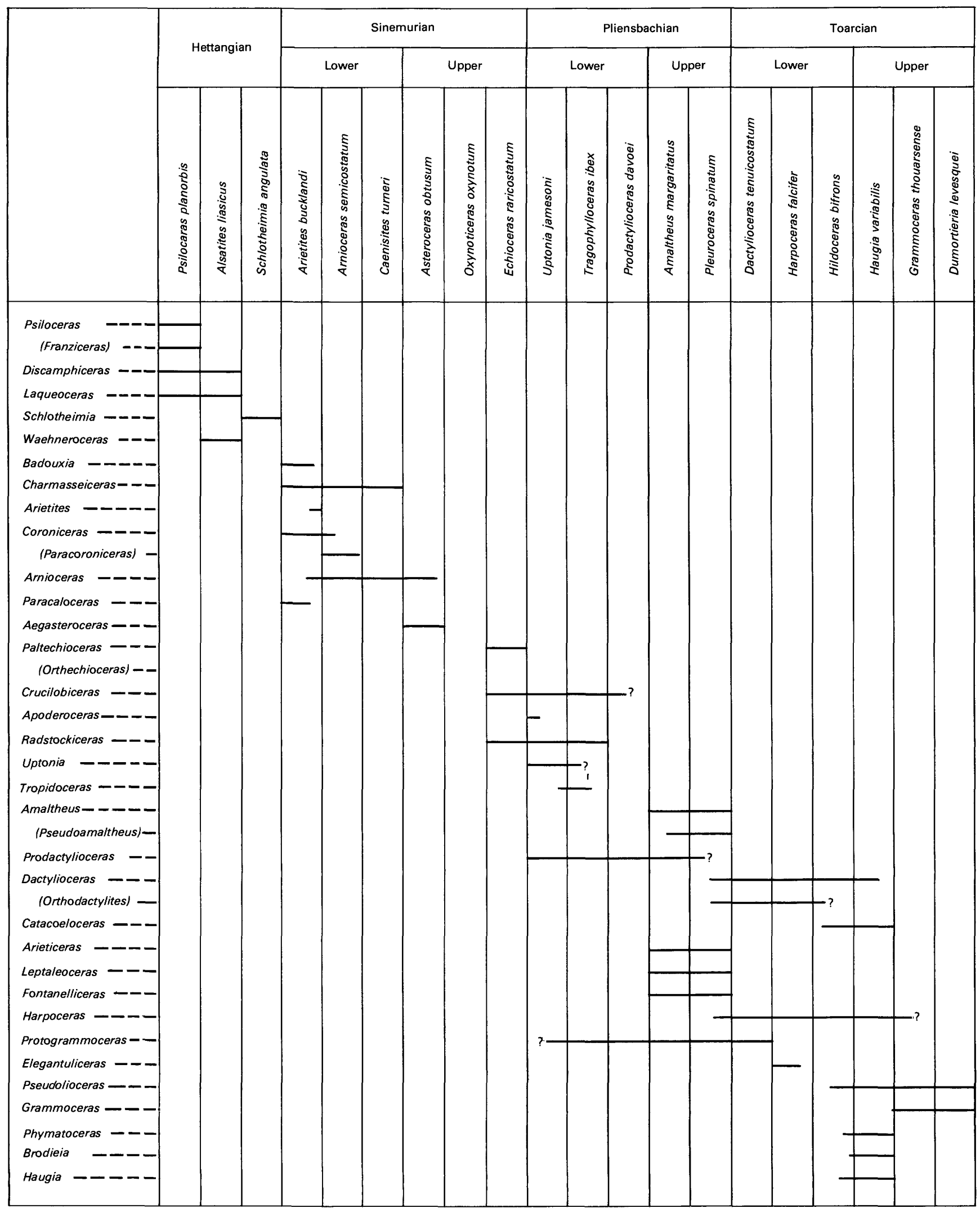

FIGURE 7.-European ranges of Early Jurassic ammonite genera present in Alaska. 


\begin{tabular}{|c|c|c|c|c|c|c|c|}
\hline 巡 & $\begin{array}{l}\mathscr{D} \\
\stackrel{\varpi}{\infty}\end{array}$ & $\begin{array}{l}\text { Northwest European } \\
\text { Ammonite zones } \\
\text { (After Dean, Donovan, } \\
\text { and Howarth, 1961) }\end{array}$ & $\begin{array}{l}\text { Arctic Coastal Plain } \\
\text { in subsurface of } \\
\text { Point Barrow- } \\
\text { Cape Simpson area }\end{array}$ & $\begin{array}{l}\text { DeLong Mountains } \\
\text { area near } \\
\text { Thetis Creek and } \\
\text { Epewik River } \\
\text { northwestern Alaska }\end{array}$ & $\begin{array}{l}\text { Ipnavik-Anaktuvuk } \\
\text { Rivers area } \\
\text { north-central Alaska }\end{array}$ & $\begin{array}{l}\text { Ignek Valley in } \\
\text { Mt. Michelson (C-4) } \\
\text { quadrangle to } \\
\text { Aichilik River in } \\
\text { northeastern Alaska }\end{array}$ & $\begin{array}{l}\text { Old Rampart } \\
\text { area in } \\
\text { east-central } \\
\text { Alaska }\end{array}$ \\
\hline \multirow{14}{*}{$\begin{array}{l}\frac{0}{n} \\
\frac{2}{3} \\
\frac{3}{5}\end{array}$} & \multirow{7}{*}{ 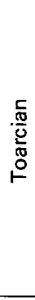 } & Dumortieria levesquej & & & & \multirow{3}{*}{ Psuedolioceras } & \\
\hline & & Grammoceras thouarsense & & & & & \\
\hline & & Haugia variabilis & & & & & \\
\hline & & Hildoceras bifrons & & Harpoceras cf. $\mathrm{H}$. & & Harpoceras cf. $H$. & \\
\hline & & \multirow{2}{*}{ Harpoceras falcifer } & & \multirow{3}{*}{$\begin{array}{l}\text { Eleganticeras } \\
\text { Dactylioceras } \\
\text { (Orthodactylites) } \\
\text { cf. D. (O.) directum }\end{array}$} & & exaratum & \\
\hline & & & \multirow{2}{*}{$\begin{array}{l}\text { Dactylioceras } \\
\text { (Orthodactylites) } \\
\text { and Catacoeloceras (?) }\end{array}$} & & & & \\
\hline & & Dactylioceras tenuicostatum & & & & & \\
\hline & \multirow{6}{*}{ 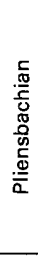 } & Pleuroceras spinatum & Amaltheus engelhardti & & & & \\
\hline & & Amaltheus margaritatus & A. margaritatus & Amaltheus & & Amaltheus & Amaltheus \\
\hline & & & A. stokesi & margaritatus & & stokesi & stokesi \\
\hline & & Prodactylioceras davoei & & & & & \\
\hline & & Tragophylloceras ibex & & & & & \\
\hline & & Uptonia jamesoni & & & $\begin{array}{r}\text { Uptonia cf. } \\
\text { U. jamesoni }\end{array}$ & & \\
\hline & \multirow{6}{*}{ 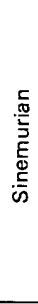 } & Echioceras raricostatum & & & & & \\
\hline \multirow{8}{*}{ 离 } & & Oxynoticeras oxynotum & & & & & \\
\hline & & Asteroceras obtusum & & & & & \\
\hline & & Caenisites turneri & & & & & \\
\hline & & Arnioceras semicostatum & $\begin{array}{l}\text { Arnioceras and } \\
\text { Coroniceras }\end{array}$ & & & & \\
\hline & & Arietites bucklandi & $\begin{array}{l}\text { Arietites, Coroniceras, } \\
\text { and Charmasseiceras }\end{array}$ & & & & \\
\hline & \multirow{3}{*}{ 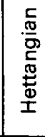 } & Schlotheimia angulata & & & & & \\
\hline & & Alsatites liasicus & Waehneroceras? & & & & \\
\hline & & Psiloceras planorbis & $\begin{array}{l}\text { Psiloceras } \\
\text { (Franziceras) }\end{array}$ & & & & $\begin{array}{l}\text { Psiloceras } \\
\text { (Franziceras) }\end{array}$ \\
\hline
\end{tabular}

FIGURE 8.-Correlation of Early Jurassic ammonite faunas in northern and east-central Alaska.

Sinemurian to late Toarcian Age (fig. 11). Other studies show (Grantz and others, 1963) that the Talkeetna Formation is overlain unconformably by beds of early Bajocian Age, that the unconformity is dated paleontologically as of latest Toarcian and earliest Bajocian Age, and that the formation is intruded by granitic rocks that may be of the same age as the unconformity.

\section{CHULITNA RIVER AREA}

The Lower Jurassic sequence exposed in the Healy (A-6) quadrangle in the south-central part of the Alaska Range has not been described. It has furnished ammonites of early Sinemurian Age at six localities and of latest Sinemurian Age at one locality (fig. 4). Most of the ammonites of early Sinemurian Age are preserved in a matrix of gray calcareous sandstone or of silty phosphatic limestone. The ammonites of latest Sinemurian Age (USGS Mesozoic loc. 31261) are preserved in a unit of massive tuffaceous argillite that apparently underlies, or is in fault contact with, siliceous argillite of Late Jurassic age (Jones and others, 1980).

\section{SHELLABARGER AND RAINY PASS AREAS}

The Lower Jurassic sequences exposed in the Talkeetna (C-6) and (B-6) quadrangles in the south-central part of the Alaska Range have not been described. In the Rainy Pass area of the Talkeetna (B-6) quadrangle, Early Jurassic fossils were obtained from gray, calcareous, fine-grained sandstone. In the Shellabarger Pass area of the Talkeetna (C-6) quadrangle, such fossils were obtained from brown siltstone and sandstone in T.28 N., R. $19 \mathrm{~W}$.

\section{KENAI PENINSULA NEAR SELDOVIA}

Lower Jurassic beds are exposed on the shore of the Kenai Peninsula for several miles southwestward from Seldovia Bay (figs. 5,14 ) in the northwestern part of the Seldovia (B-15) quadrangle. They consist of interbedded tuff, agglomerate, sandstone, shale, and limestone that probably are 2,000-3,000 feet (610-915 m) thick, according to Martin (1926, p. 135). The same sequence, based on unpublished field studies by Don Miller and the author for the U.S. Geological Survey in 1948, includes a thick middle unit consisting of unfossiliferous red lava, 


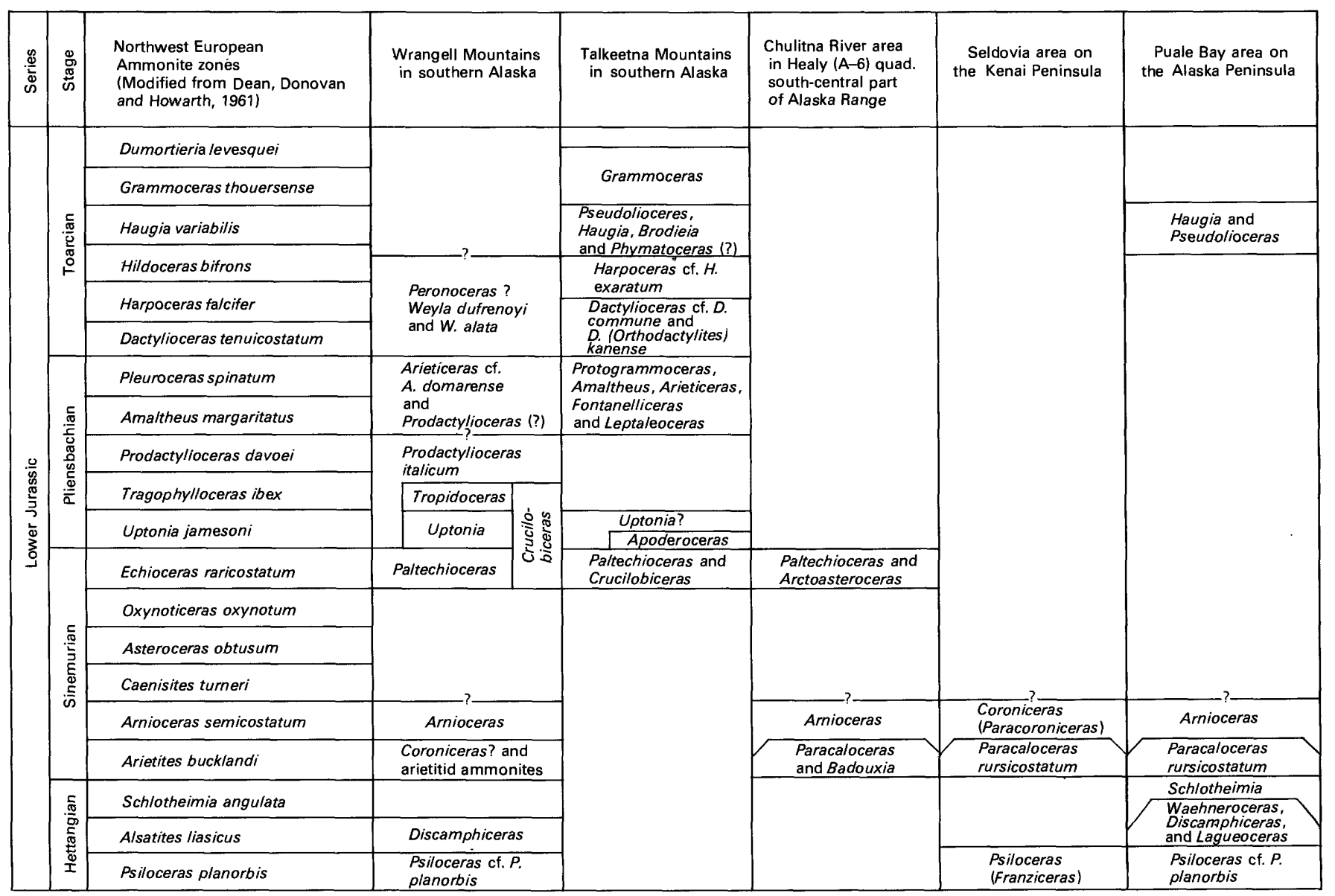

FIGURE 9.-Correlation of Early Jurassic ammonite faunas in southern Alaska.

green lava, tuff, and agglomerate overlain and underlain by units consisting of green sandstone, tuffaceous sandstone, tuff, agglomerate, and gray limestone. Fossils occur in limestone, tuff, and sandstone beds in both the lower and upper units. The ammonites present are of Hettangian and early Sinemurian Age.

This Lower Jurassic sequence, on the basis of recent studies by Kelley (1978, p. 2356), is about 4,000 m thick and consists mainly of pyroclastic breccias and tuff. It is divisible into three mappable lithofacies, from bottom to top: (1) a "graded pyroclastic lithofacies" deposited below wave base; (2) a "pyroclastic debris flow and turbidite lithofacies" deposited below wave base; and (3) a "reworked volcaniclastic lithofacies" marked basally by fairly thick coal beds but deposited partly under tidal influences. Kelley noted that fossils occur in all three units.

\section{PUALE BAY-ALINCHAK BAY AREA}

The Lower Jurassic sequence in the Puale Bay area, as described by Imlay and Detterman 1977), is at least 1,850 feet $(550 \mathrm{~m})$ thick. At its base is a limestone unit about 780 feet $(238 \mathrm{~m})$ thick that in its lower part contains ammonites of Hettangian Age and that rests con- formably on limestone of latest Triassic age. The overlying 1,040 feet $(317 \mathrm{~m})$ of beds consists mostly of massive tuffaceous conglomeratic sandstone but includes some siltstone and limestone. These beds contain ammonites of early Sinemurian Age. This sandstone is overlain sharply by sandy siltstone of the Kialagvik Formation, which is mostly of early to middle Bajocian Age. Its lowest beds, however, are of middle to early late Toarcian Age, as shown by the presence of Haugia about 30 feet $(9 \mathrm{~m})$ above its base. The Toarcian probably also includes some of the overlying $320-370$ feet (98-113 m) of siltstone between this occurrence of Haugia and the lowest occurrence of Pseudolioceras whiteavesi (White) of late early Bajocian Age (figs. 9, 12). The sharp contact at the base of the Kialagvik Formation probably represents a fault rather than an unconformity.

\section{AGES AND CORRELATIONS}

\section{HETTANGIAN AMMONITES}

Hettangian ammonites have been found in the subsurface of northern Alaska, in the Old Rampart area of 


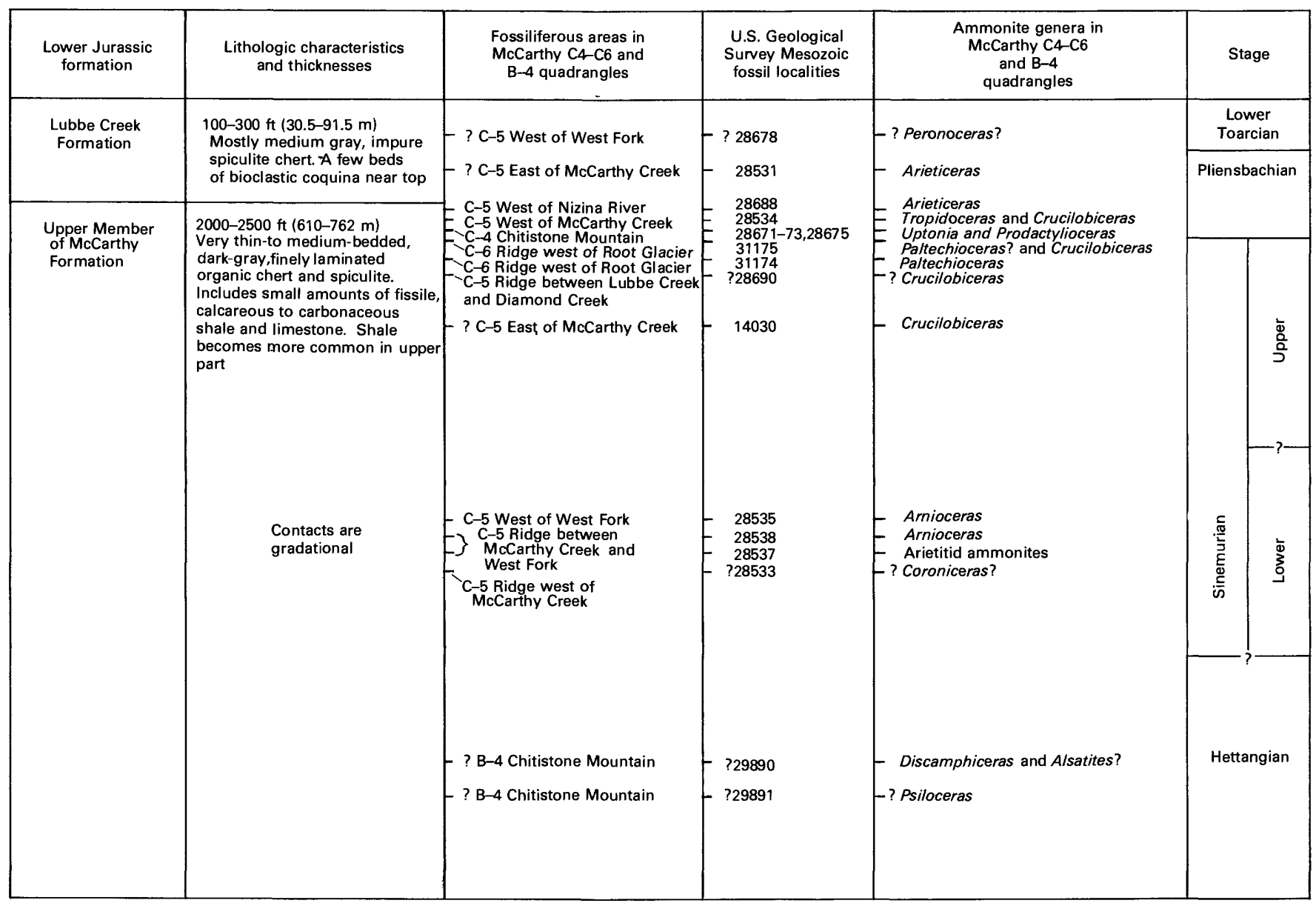

FIGURE 10.-Occurrences and ages of ammonites present in the Lower Jurassic beds exposed in the Wrangell Mountains, southern Alaska.

northern east-central Alaska, in the Wrangell Mountains of southern Alaska, in the Seldovia area of the Kenai Peninsula and in the Puale Bay-Alinchak Bay and Wide Bay areas of the Alaska Peninsula (figs. 8-10,12).

The earliest Hettangian is represented in all these areas, except for Wide Bay, either by a fairly smooth species of Psiloceras or by the ribbed subgenus P. (Franziceras) similar to $P$. (F.) ruidum (Buckman). The middle Hettangian, probably correlative with the Alsatites liasicus zone of Europe, is characterized by Waehneroceras at Wide Bay, by Waehneroceras, Discamphiceras, and Laqueoceras at Puale Bay, by Discamphiceras in the Wrangell Mountains, and questionably by Waehneroceras in the Arctic Coastal Plain near Point Barrow (fig. 8). The late Hettangian, correlative with the Schlotheimia angulata Zone, is represented by one specimen of Schlotheimia in the Puale Bay area. All these genera are represented by species that are closely similar to, and probably in part identical with, species described from western Europe. Evidently the succession of Hettangian ammonite genera and species in Alaska is the same as in western Europe.

\section{SINEMURIAN AMMONITES}

Sinemurian ammonites have been found in northern Alaska only in the subsurface of the Point Barrow-Cape Simpson area (fig. 7). In southern Alaska, such ammonites occur in five areas from the Wrangell Mountains on the east to Puale Bay on the west (figs. 10-12). The lower Sinemurian zones of Arietites bucklandi and Arnioceras semicostatum are represented in all these areas except in the Talkeetna Mountains, where beds of that age are not exposed. The uppermost Sinemurian (Echioceras raricostatum Zone) is represented in the Wrangell and Talkeetna Mountains and in the Chulitna River area of the Alaska Range. The remainder of the Sinemurian (Caenisites turneri, Asteroceras obtusum, and Oxynoticeras oxynotum Zones) has not been identified by ammonites anywhere in Alaska but is probably represented by poorly fossiliferous beds in the Arctic Coastal Plain, in the Wrangell Mountains, and possibly elsewhere. 


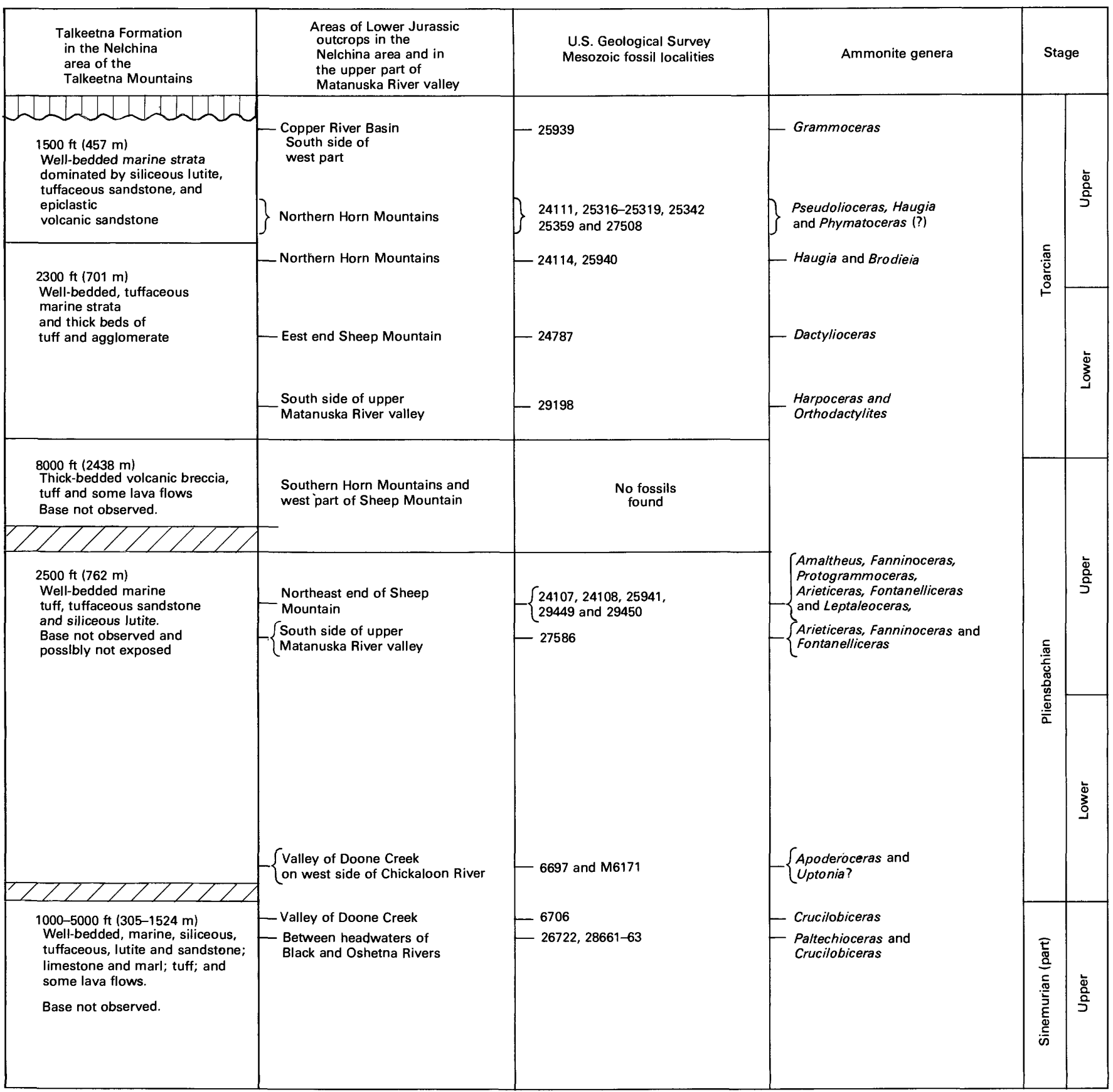

Figure 11.-Occurrences and ages of Early Jurassic ammonites present in the Nelchina area of the Talkeetna Mountains and in the upper part of the Matanuska Valley, southern Alaska.

The lowermost Sinemurian (Arietites bucklandi Zone) in the Arctic Coastal Plain is represented by $A$. cf. $A$ bucklandi (Sowerby) in the Avak test well no. 1 at the depth of 1,836 feet $(560 \mathrm{~m})$. It is represented by Charmasseiceras in the South Barrow test well no. 3 at depths of 2,412 and 2,419.5 feet (735 and $737 \mathrm{~m}$ ) and in the South Barrow test well no. 12 at depths of 2,056, $2,061,2,061.5$ and 2,068 feet $(627-630 \mathrm{~m})$. The Sinemurian Age of Charmasseiceras in the South Bar- row test well no. 3 is attested by the presence of Coroniceras sp. C (pl. 4, fig. 14) in the same well at the depth of 2,470 feet $(753 \mathrm{~m})$.

The earliest Sinemurian in the Wrangell Mountains is probably represented by fragmentary arietitid ammonites that occur a little below beds containing $A r$ nioceras (figs. 9, 10). Beds of earliest Sinemurian Age in the Chulitna River, Seldovia, and Puale Bay areas are represented by Paracaloceras rursicostatum Frebold 


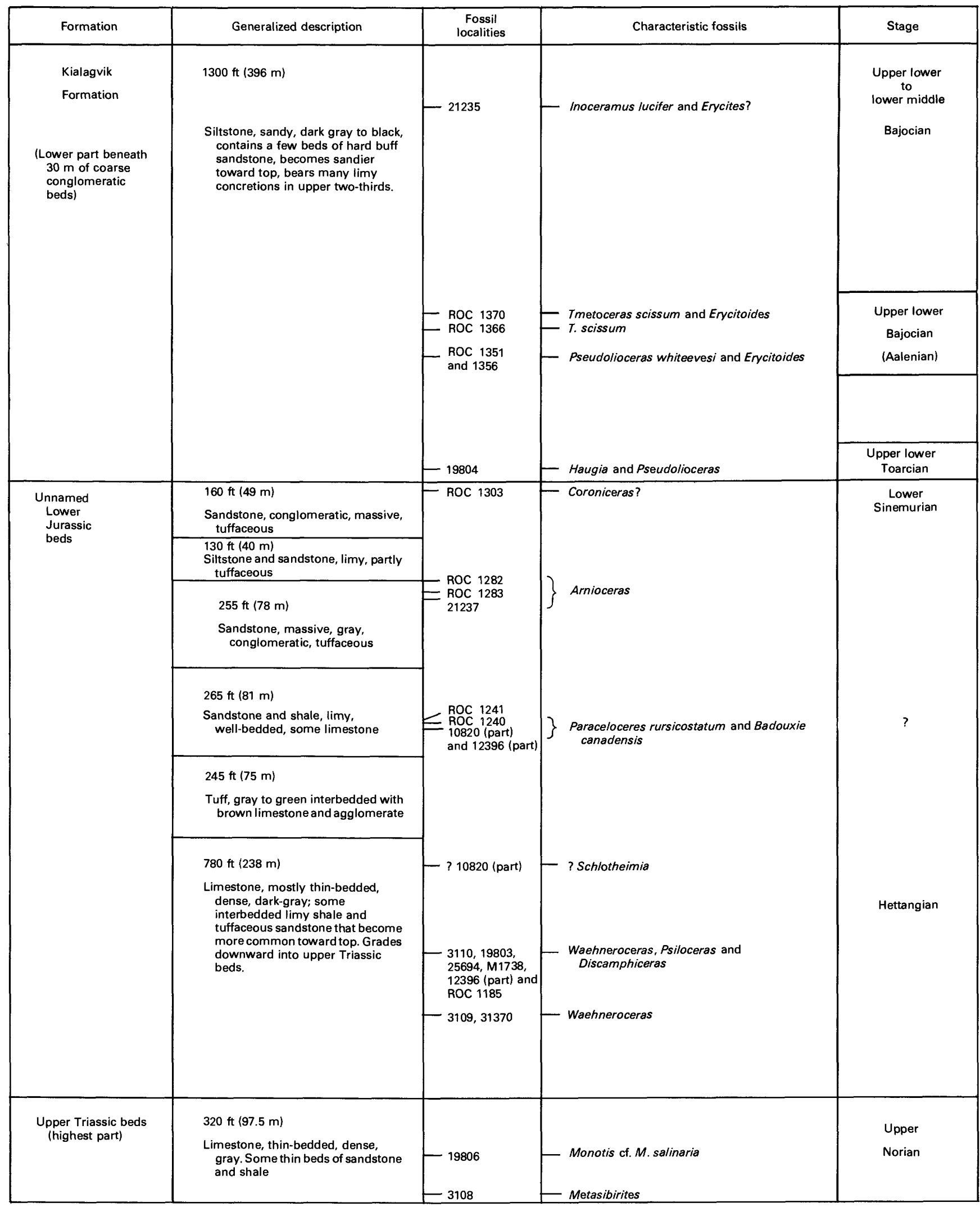

FIGURE 12,-Occurrences and ages of ammonites present in unnamed Lower Jurassic beds on northeast side of Puale Bay, Alaska Peninsula. 


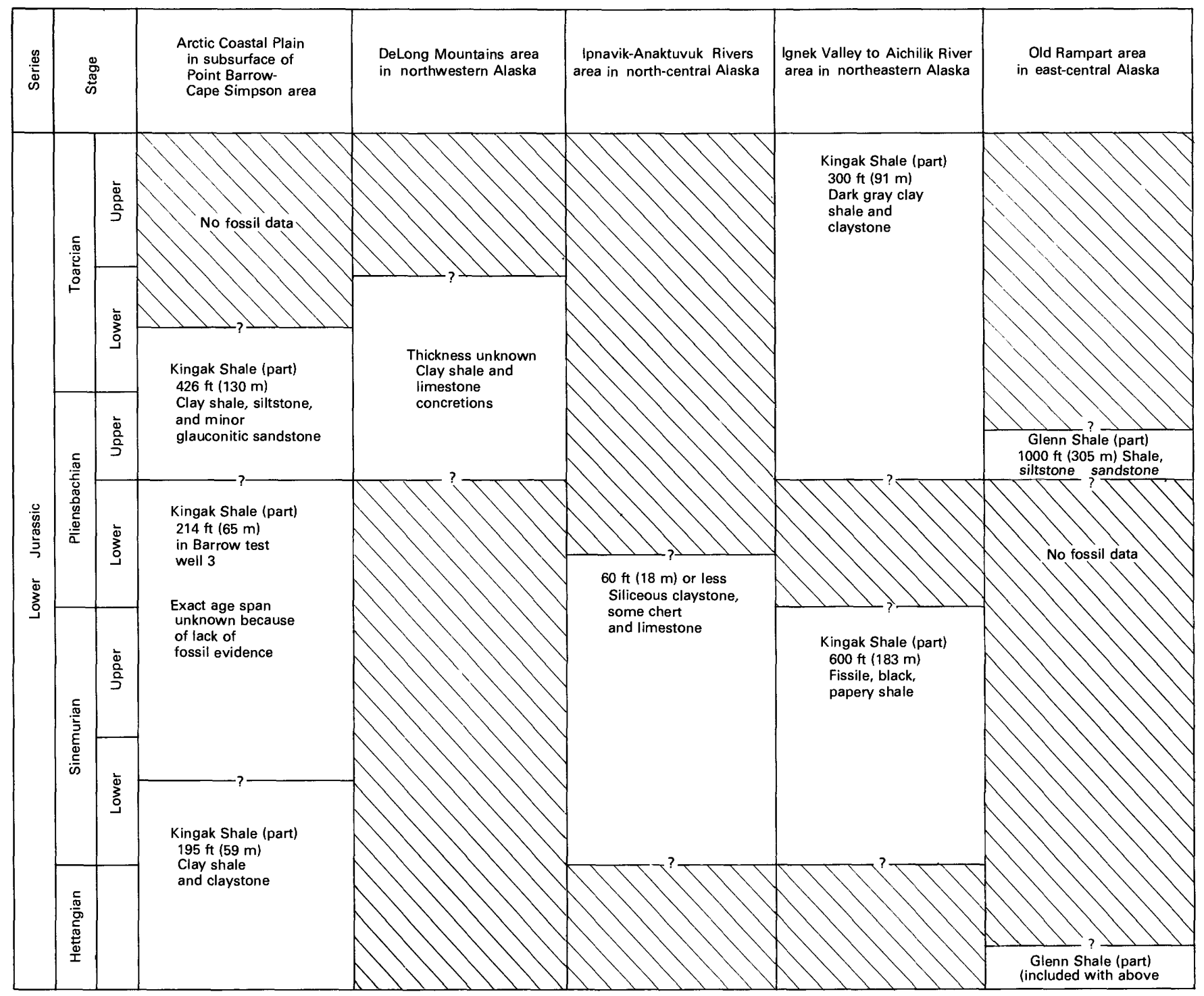

FIGURE 13.-Correlation of Lower Jurassic rocks in northern and east-central Alaska.

(1967b, p. 26, pl. 7, figs. 1, 2; pl. 9, fig. 1). That species in the Chulitna River area occurs with Psiloceras canadense Frebold (1951, p. 3, pl. 6, figs. 1-6; pl. 2, fig. 1; pl. 3, fig. 1) which is now assigned to Badouxia Guex and Taylor (1976, p. 525). Those species of Paracaloceras and Badouxia in association with others in north-central British Columbia were tentatively assigned a middle Hettangian Age by Frebold (1967b, p. 31). Later studies by Guex and Taylor (1976) show, however, that Paracaloceras and Charmasseiceras in many parts of the world are associated with ammonites of early Sinemurian Age as young as the Arnioceras semicostatum zone. Such an age for those genera in British Columbia is confirmed by their association with Vermiceras multicostatum (Frebold), which genus is characteristic of the earliest Sinemurian.
The lower Sinemurian zone of Arnioceras semicostatum is represented in four areas in Alaska by a finely ribbed species of Arnioceras similar to $A$. densicosta (Quenstedt). In the Seldovia area, the same zone is probably represented by the subgenus Coroniceras (Paracoroniceras), which is not known in younger zones.

The next three younger Sinemurian zones, although not identified faunally, could be represented by poorly fossiliferous beds, or in part by the presence of the genus Arnioceras, which in Europe ranges upward through most of the Asteroceras obtusum Zone.

The uppermost Sinemurian zone of Echioceras raricostatum is represented in both the Wrangell and Talkeetna Mountains by Paltechioceras cf. $P$. harbledownense (Crickmay) in association with species of Crucilobiceras that closely resemble described species 


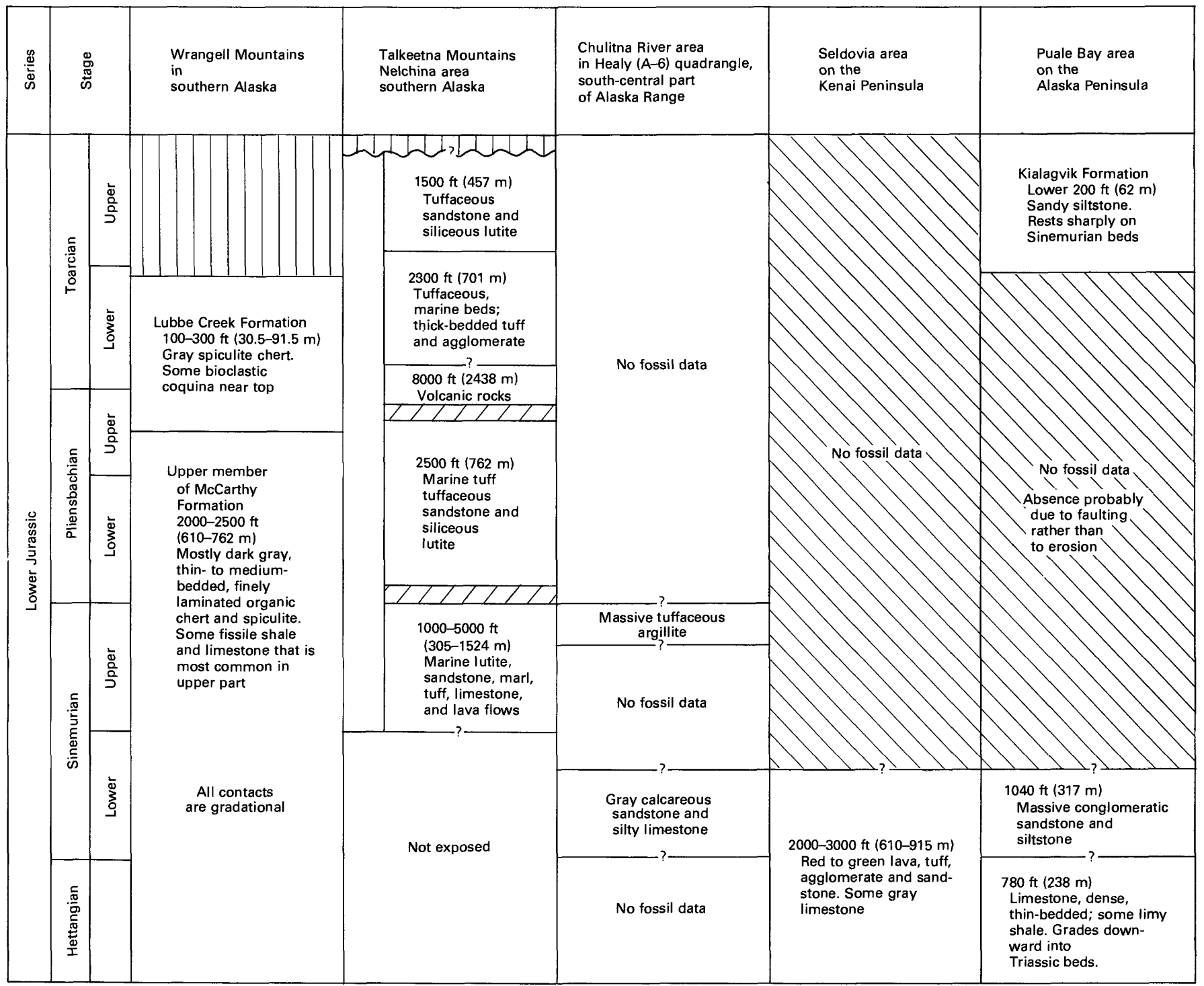

Figure 14.-Correlation of Lower Jurassic rocks in southern Alaska.

from western Europe. Paltechioceras occurs also in the Chulitna River area in association with Arctoasteroceras jeletzkyi Frebold, whose type specimens in arctic Canada were collected along with Oxynoticeras oxynotum (Quenstedt) below beds containing echioceratid ammonites. This shows that Arctoasteroceras occurs in the highest two zones of the Sinemurian.

Other mollusks of some age significance during Sinemurian time include the bivalves Otapiria tailleuri Imlay and Entolium? semiplicatum Hyatt. Of these, $O$. tailleuri in the subsurface of northern Alaska occurs in the South Barrow test well No. 3 at depths of 2,412-2,417 feet $(735 \mathrm{~m})$ and in the South Barrow test well No. 12 at depths of 2,055-2,078.5 feet (627-634 m). Comparisons with the ammonite occurrences in those test wells (table 2) show clearly that $O$. tailleuri is associated with ammonites of early Sinemurian Age, occurs above ammonites of Hettangian Age, and has its highest occurrence at 214 feet $(65 \mathrm{~m})$ below ammonites of late Pliensbachian Age.

Otapiria tailleuri Imlay has been found in outcrops in northern Alaska in a fairly thin unit of fissile clay shale that extends from the Ipnavik River eastward at least 200 miles $(320 \mathrm{~km}$ ) to the Kavik River (Imlay, 1967, p. B6, B7; Imlay and Detterman, 1973, p. 12; Detterman and others, 1975, p. 44). Apparently it does not occur throughout the entire thickness of the shale in all areas. It does occur in the same rock slabs as some crushed, generically undeterminable ammonites (Imlay, 1967, p. B6). It does not occur on the same slabs as some ammonites of early Pliensbachian Age that are described herein as Uptonia cf. U. jamesoni (J. de C. Sowerby) and 
TABLE 2.-Early Jurassic ammonites from well cores in northern Alaska

\begin{tabular}{|c|c|c|c|c|c|}
\hline \multirow[b]{2}{*}{ Genus and species } & \multicolumn{4}{|c|}{ Depths in test wells, in feet, from which fossils were obtained } & \multirow{2}{*}{ Stages } \\
\hline & Simpson test well 1 & Avak test well 1 & $\begin{array}{l}\text { South Barrow } \\
\text { test well } 3\end{array}$ & $\begin{array}{c}\text { South Barrow } \\
\text { test well } 12\end{array}$ & \\
\hline $\begin{array}{l}\text { Dactylioceras (Orthodactylites) } \\
\text { cf. D. (O.) kanense McLearn. }\end{array}$ & 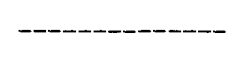 & - & 2,016 & ........-...- & Lower Toarcian. \\
\hline D. $\mathrm{sp}$ & $\ldots$ & $\ldots$ & $72 ; 2,017 ; 2,018$ & 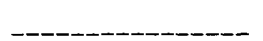 & Do. \\
\hline Catacoeloceras sp. juv & - & - & 2,063 & - & Do. \\
\hline A maltheus margaritatus (Montfort) & - & - & 2,$099 ; 2,111$ & - & Upper \\
\hline & & & & & Pliensbachian. \\
\hline A. cf. A. margaritatus (Montfort) & & & 2,107 & & Do. \\
\hline A. stokesi (J. Sowerby) & --n-- & - & 2,$193 ; 2,198$ & & Do. \\
\hline A. cf. A. stokesi (J. Sowerby) & - & - & 2,186 & & Do. \\
\hline$A . \mathrm{sp}$ & 5,$677 ; 5,680 ; 5,691$ & - & $2,069-2,184$ & 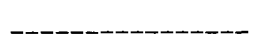 & Do. \\
\hline $\begin{array}{l}\text { A. (Pseudoamaltheus) engelhardti } \\
\text { (d'Orbigny). }\end{array}$ & - & - & 2,090 & 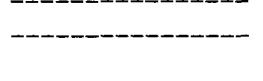 & Do. \\
\hline Arietites cf. A. bucklandi (Sowerby) & & 1,836 & -_- & & Lower Sinemurian. \\
\hline Coroniceras sp. B & & & ב-_-_ & $1,987.4-1,987.6$ & Do. \\
\hline 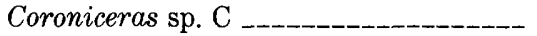 & & & 2,470 & & Do. \\
\hline Arnioceras sp. juv __-_-_________-_-_-_ & _._-_._-_._-_-_-_ & _-_-_-_-_-_-_-_-_. & ....- & 2,056 & Do. \\
\hline Charmasseiceras sp. _-_______________-_._- & & & & 2,056 & Do. \\
\hline C. cf. C. marmoreum (Oppel) & _._._._._._._._. & 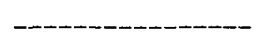 & 2,$412 ; 2,419.5$ & $2,061.5 ; 2068$ & Do. \\
\hline Psiloceras (Franziceras) sp _._. & -_- & - & 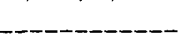 & $2,170.5$ & Hettangian. \\
\hline Waehneroceras? sp & - & & & $2,181.5$ & Do. \\
\hline
\end{tabular}

Uptonia sp. (pl. 9, figs. 9-11, 17). These occurrences and associations in both surface and subsurface rocks show that the range of $O$. tailleuri is probably only Sinemurian and that it may not be younger than early Sinemurian.

The other pelecypod that is useful in dating Sinemurian rocks is Entolium? semiplicatum Hyatt. (See Crickmay, 1933, p. 52, pl. 14, figs. 4-7.) That species to date has been found in Alaska only in the Wrangell Mountains, where it is common in dark-gray to black fissile shale at the top of the McCarthy Formation. In that shale, which is similar lithologically to the fissile shale in which Otapiria occurs in northern Alaska, it is associated with the ammonites Paltechioceras, (USGS Mesozoic locs. 30139 and 31174) and Crucilobiceras cf. C. crucilobatum Buckman (USGS Mesozoic loc. 14030) of latest Sinemurian Age. It has been found elsewhere in the Pacific Coast region on the North Fork of the American River in eastern California (Crickmay, 1933, p. 52; Imlay, 1968, p. C7) in association with Crucilobiceras. It is closely similar to and possibly within the range of variation of Entolium balteatum Crickmay (1928, p. 62, pl. 4e-g) from Parson Bay in British Columbia. That species is associated with Paltechioceras harbledownese (Crickmay) (1928, p. 61, pl. 4a-d), which is probably represented at the top of the McCarthy Formation in Alaska, (pl. 4, figs. 15-17, 22).

These data concerning Entolium? semiplicatum and Otapiria tailleuri show that both are of Sinemurian Age and both occur in similar fissile shale. The fact that they have never been found associated in the same bed suggests, therefore, that they are of slightly different ages, or lived in somewhat different marine environments, or were separated by a land barrier.

\section{PLIENSBACHIAN AMMONITES}

Pliensbachian ammonites have been found in both the subsurface and surface in northern Alaska, in the Old Rampart area in east-central Alaska, and in the Wrangell and Talkeetna Mountains in the eastern part of southern Alaska. They probably occur in the Yakutat District in the northern part of southeastern Alaska (figs. 8-11).

In northern and east-central Alaska, most Pliensbachian ammonite occurrences consist of Amaltheus of late Pliensbachian Age. In the South Barrow test well No. 3, Amaltheus is represented by the same species in the same succession as in northwestern Europe (table 2). The only other ammonite of Pliensbachian Age found in northern Alaska consists of a single large specimen of Uptonia similar to $U$. jamesoni (J. de C. Sowerby) from an outcrop on Lisburne Ridge (USGS Mesozoic loc. 29774) between the Ipnavik and Etivluk Rivers in the Howard Pass quadrangle. This specimen is good evidence that the beds in which it occurs are of earliest Pliensbachian Age. In addition Uptonia, rather than Crucilobiceras, may be represented in the Ipnavik-Anatuvuk River area by several crushed molds (pl. 9, figs. 9-11) (USGS Mesozoic locs. 29281, 29282, and 29775) that resemble the inner whorls of the large specimen of Uptonia.

The Pliensbachian ammonite successions in the Wrangell and Talkeetna Mountains in southern Alaska contrast with those in northern Alaska by being fairly complete, by containing some ammonites that are closely similar to taxa in the Mediterranean region, and by the genus Amaltheus being represented by only two specimens from a single locality. In the Wrangell Mountains the Uptonia jamesoni zone is represented by $U$. cf. 
$U$. dayiceroides Mouterde, which was collected with Prodactylioceras italicum (Fucini) less than 100 feet (30 m) below the upper contact of the upper member of the McCarthy Formation (USGS Mesozoic locs. 28671-28673, 28175). Most of the Tragophylloceras ibex zone is represented by Tropidoceras actaeon (d'Orbigny), which was collected with Crucilobiceras cf. C. pacificum Frebold about $1 \mathrm{~m}$ below the upper contact of the McCarthy Formation (USGS Mesozoic loc. 28534). The uppermost Pliensbachian is represented by Arieticeras at the top of the McCarthy Formation (USGS Mesozoic loc. 28688) and in the overlying Lubbe Creek Formation (USGS Mesozoic loc. 28531).

In the Talkeetna Mountains (figs. 9, 11), the Uptonia jamesoni zone is represented by a well-preserved specimen of Apoderoceras cf. A. subtriangulare (Young and Bird) and a fragmentary specimen that probably represents Uptonia. The next two younger zones of western Europe are not represented by fossils, but the uppermost two Pliensbachian zones are represented by Leptaleoceras pseudoradians (Reynes), Fontanelliceras cf. $F$. fontanellense (Gemmellaro) Arieticeras cf. $A$. domarense (Meneghini), Protogrammoceras cf. $P$. paltum (Buckman), P. cf. P. argutum (Buckman), Fanninoceras kunae McLearn, and Amaltheus cf. A. stokesi (J. Sowerby).

\section{TOARGIAN AMMONITES}

The Toarcian is not well represented by ammonites in Alaska except in the Talkeetna Mountains (figs. 8, 9, 11). In the subsurface of the arctic Coastal Plain, only the lowermost Toarcian (characterized by Dactylioceras) has been identified. In the DeLong Mountains, that genus is succeeded upward by Harpoceras cf. $H$. exaratum (Young and Bird), which succession accounts for about two-fifths of Toarcian time. The same species of Harpoceras was obtained in northeastern Alaska on a tributary of the Sadlerochit River (USGS Mesozoic loc. 22081). Near Red Hill on Ignek Creek in the Mt. Michelson (C-4) quadrangle, was obtained Pseudo- lioceras cf. $P$. compactile (Simpson) and $P$. cf. $P$. lythense (Young and Bird) of late Toarcian Age at USGS Mesozoic loc. 23772 (Imlay, 1955, p. 89 pl. 12, figs. 17, $18,20,21)$.

In the Talkeetna Mountains of southern Alaska, the lower Toarcian is represented by a similar sequence of Dactylioceras and Harpoceras as in northern Alaska. The lower upper Toarcian is represented by Haugia, Brodieia, and Pseudolioceras, which genera in association are good evidence for correlation with the Haugia variabilis Zone of western Europe. Still higher occurs Grammoceras, a genus that in western Europe occurs only in the uppermost two zones of the Toarcian.

In the Puale Bay area, the middle to lower upper Toarcian is represented by Haugia and Pseudolioceras.

\section{AMMONITE FAUNAL SETTING}

No distinct ammonite faunal province existed in Alaska during most of Early Jurassic time, as shown by the remarkable resemblance of most of the ammonites present to those elsewhere in the world. During late Pliensbachian time, a close tie with northern Europe is shown by the presence of Amaltheus in northern Alaska and in the Talkeetna Mountains of southern Alaska. At the same time, a close tie with the Mediterranean region is shown by the presence of Arieticeras in the Wrangell Mountains and of Arieticeras, Leptaleoceras, Fontanelliceras, and Protogrammoceras in the Talkeetna Mountains but not farther north. These occurrences of Arieticeras and Leptaleoceras in southern Alaska are not much farther north than their northernmost known occurrences in the southern Yukon (Frebold, 1970, p. 446).

\section{GEOGRAPHIC DISTRIBUTION}

The geographic occurrences of Early Jurassic ammonites from Alaska described herein are shown on figures 1-6 and tables 3-6. Detailed descriptions of the occurrences are given in table 7 . 
TABLE 3.-Geographic distribution of Early Jurassic ammonites from outcrops in northern and east-central Alaska [Quadrangle occurrences are listed in table 7. Numbers 5-15 are keyed to area numbers in figure 1. Higher numbers are USGS Mesozoic locality numbers]

\begin{tabular}{|c|c|c|c|c|c|c|c|c|c|}
\hline \multirow{2}{*}{\multicolumn{2}{|c|}{$\begin{array}{c}\text { Northwestern Alaska } \\
\text { Delong Mountains }\end{array}$}} & \multirow{2}{*}{\multicolumn{3}{|c|}{$\begin{array}{l}\text { North-central } \\
\text { Alaska }\end{array}$}} & \multirow{2}{*}{\multicolumn{3}{|c|}{$\begin{array}{l}\text { Northeastern } \\
\text { Alaska }\end{array}$}} & \multirow{2}{*}{\multicolumn{2}{|c|}{$\begin{array}{c}\text { East-central Alaska } \\
\text { Old Rampart area }\end{array}$}} \\
\hline & & & & & & & & & \\
\hline \multicolumn{2}{|l|}{ Clay shale } & \multicolumn{3}{|c|}{ Claystone } & \multicolumn{3}{|c|}{ Kingak shale } & \multicolumn{2}{|l|}{$\begin{array}{l}\text { Glenn shale } \\
\text { (in part) }\end{array}$} \\
\hline 5 & 6 & 7 & 8 & 9 & \begin{tabular}{l|l}
10 & 1
\end{tabular} & \begin{tabular}{l|l|}
11 & 12
\end{tabular} & \begin{tabular}{|l|}
13 \\
\end{tabular} & 14 & 15 \\
\hline 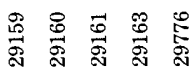 & 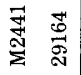 & 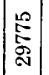 & 营 & 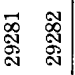 & 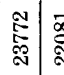 & $\begin{array}{lll}0 \\
0\end{array}$ & 嚆 & 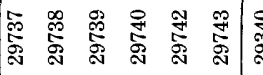 & 㞼 \\
\hline
\end{tabular}

Psiloceras sp _._. $P . ? \mathrm{sp}$ P. (Franziceras) sp _._-___$P .(F$.$) cf. P .(F$.$) ruidum (Buckman)$ Uptonia cf. U. jamesoni (J. de C. Sowerby) _Uptonia? sp ___

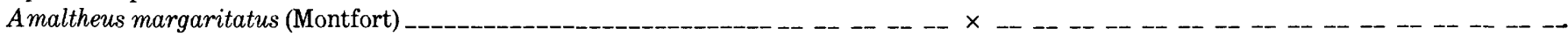
A. stokesi (J. Sowerby) _____ A. cf. A. stokesi (J. Sowerby) A. $\mathrm{sp}$ Dactylioceras (Orthodactylites) cf. $D$. (O.) directum Buckman

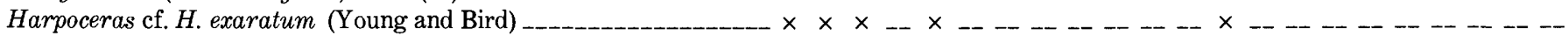
Eleganticeras sp. juv

Pseudolioceras cf. P. compactile (Simpson) $P$. cf. P. lythense (Young and Bird) _. 
TABLE 4.-Geographic distribution of Early Jurassic ammonites in the Yakutat area and in the Wrangell Mountains in southern Alaska [Quadrangles are listed in table 7. Numbers 16-19 are keyed to generalized area numbers in figure 1. Higher numbers are USGS Mesozoic locality numbers]

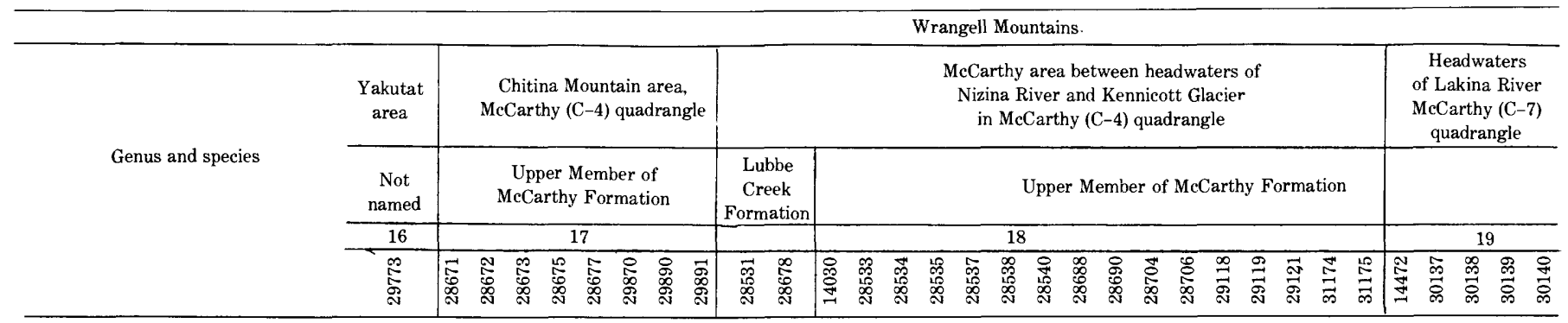

Psiloceras cf. P. planorbis (J.

de C. Sowerby)

Discamphiceras $\mathrm{sp}$

Arnioceras cf. A. densicosta

(Quenstedt)

Coroniceras? sp _..--_---_--

Arietitid genus undetermined

Paltechioceras cf. $P$. harble-

$P . ? \mathrm{sp}$

Crucilobiceras cf. C. crucilo-

batum Buckman -

C. cf. C. pacificum Frebold _-_-_

C. sp

Uptonia cf. U. dayiceroides

Mouterde

Uptonia? sp. A

Tropidoceras actaeon

(d'Orbigny)

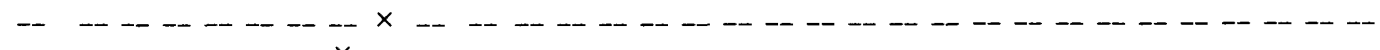

_.

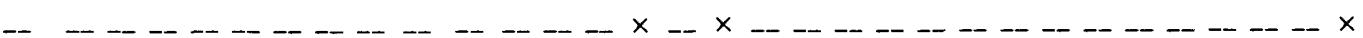

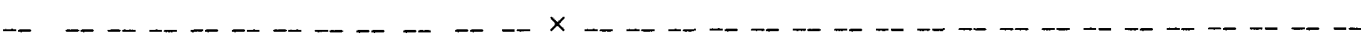

-

-- - - - - - - - - - - - - - - - -

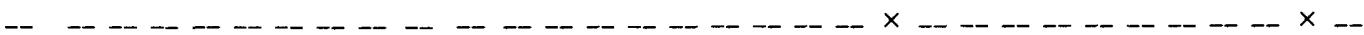

-

-1
-

_-

Prodactylioceras italicum

italicum (Fucini)

P. cf. P. italicum fucini

R. Fischer

$P . ? \mathrm{sp}$

Arieticeras cf. A. domarense

(Meneghini)

A. cf. A. algovianum (Oppel) --

A. $\mathrm{sp}$

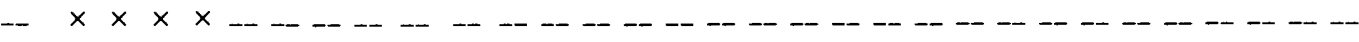

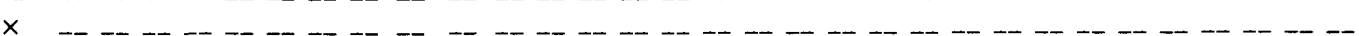

--

- $\times$ -

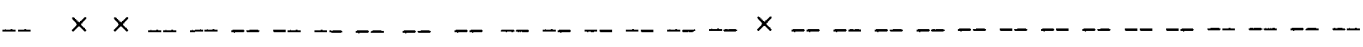

-_

-

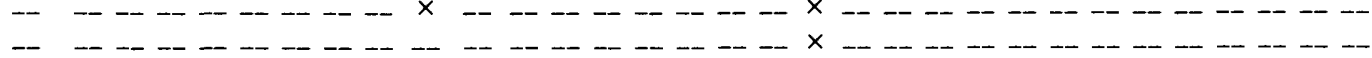

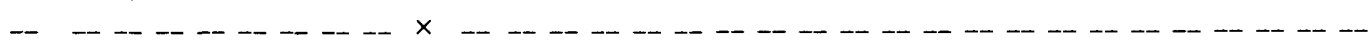


TABLE 5.-Geographic distribution of Early Jurassic ammonites in the Talkeetna Mountains in southern Alaska [Quadrangle occurrences are listed in table 7. Numbers 20-23 are keyed to generalized area numbers in figure 1. Higher numbers are USGS Mesozoic locality numbers]

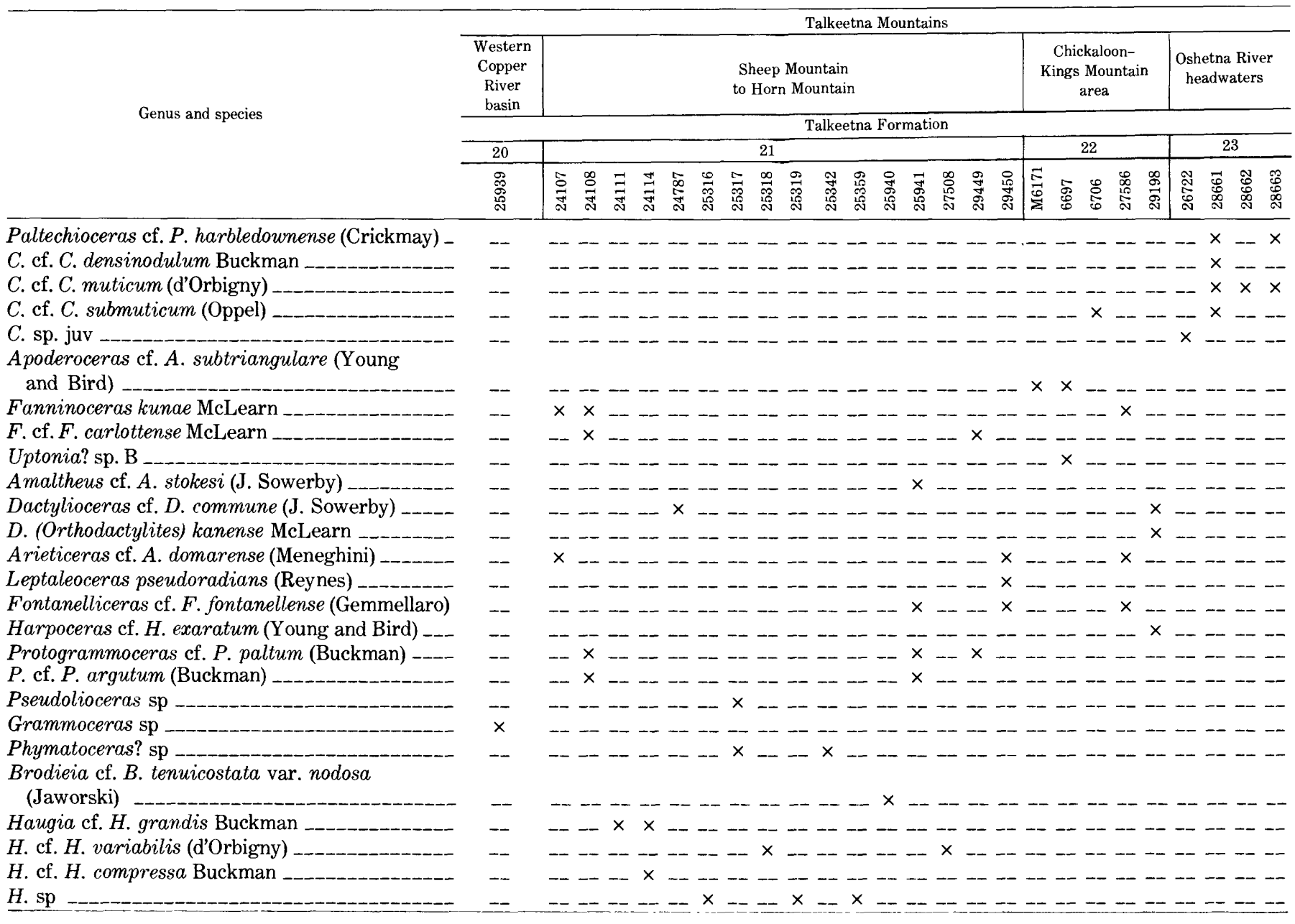


TABLE 6.-Geographic distribution of Early Jurassic ammonites in the Chulitna River area of the Alaska Range, in the Seldovia [Quadrangle occurrences are listed in table 7.

\begin{tabular}{|c|c|c|c|}
\hline \multirow{5}{*}{ Genus and species } & \multicolumn{2}{|c|}{ South-central part of Alaska Range } & Kenai Peninsula \\
\hline & $\begin{array}{l}\text { Chulitna River area } \\
\text { Healy A-6 quadrangle }\end{array}$ & \begin{tabular}{|c|} 
Talkeetna \\
B-6 and \\
C-6 quads- \\
rangles \\
\end{tabular} & Seldovia area \\
\hline & \multicolumn{2}{|l|}{$\begin{array}{l}\text { Sandstone, siltstone, } \\
\text { limestone, and argillite }\end{array}$} & Volcanic rocks, sandstone, limestone \\
\hline & 24 & 25 & 26 \\
\hline & 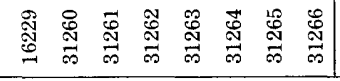 & 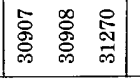 & 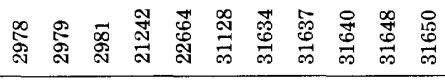 \\
\hline ytoceras $\mathrm{sp}$ & $\ldots \ldots \ldots \ldots$ & $\ldots \ldots \ldots$ & - \\
\hline Psiloceras cf. P. planorbis (J. de C. Sowe & & & -. - - - - - - - - - - - - - - - \\
\hline P. (Franziceras) cf. $P$. (F.) ruidum (Buck & - - - - - - - - - - - - & -. - - - - & $\ldots \ldots \times \ldots \ldots \ldots+\ldots$ \\
\hline Discamphiceras cf. D. toxophorum (Buck & -- -- -- -- -- -- - - - & ---- & -- - - - - - - - - - - - - - - - - \\
\hline $\begin{array}{l}\text { Laqueoceras cf. L. sublaqueus (Waehner) } \\
\text { Waehneroceras cf. W. tenerum (Neumayr) }\end{array}$ & ב- & ---- & 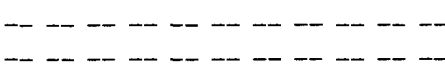 \\
\hline$W$. cf. W. portlocki (Wright) & 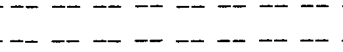 & -- - & 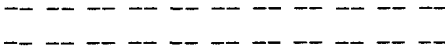 \\
\hline Scholtheimia sp & 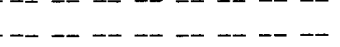 & $-\ldots-\ldots$ & - - - - - - - - - - - - \\
\hline Badouxia canadensis (Frebold) & - _. - - - - - & - - - - - & - - - - _ - - - - - - - - - - - \\
\hline B. columbiae (Frebold) ___ & $\ldots+\ldots+\ldots+\ldots$ & ---- & -- -- - - - - - - - - - - - - - \\
\hline Charmasseiceras cf. C. marmoreum (Opp & -. - - - - - - - - - -- & ---- & $-x_{-\ldots}+\ldots-\ldots \times \ldots$ \\
\hline Coroniceras sp. A & - - - - - - - - - - - - & $\ldots \times \ldots$ & -- - - - - - - - - - - - - - \\
\hline Coroniceras? sp ______________ & - - - - - - - - - - - - & ---- & $\times \ldots-\ldots+\ldots \times-x_{--}$ \\
\hline C. (Paracoroniceras) sp & -- -- - - - - - - - - - & ---- & -- - - - - $\times$ x - - - - $\times$ \\
\hline Arnioceras cf. A. densicosta (Quenstedt) & $\times \times-x \times-x_{--}$ & ---- & 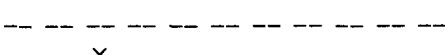 \\
\hline Paracaloceras rursicostatum Frebold & $---------x$ & $-\cdots-\cdots$ & 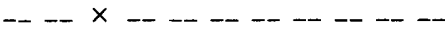 \\
\hline $\begin{array}{l}\text { Arietitid genus undetermined } \\
\text { Arctoasteroceras jeletzkyi Frebold }\end{array}$ & 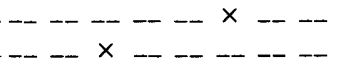 & $\times-x_{-} \times-$ & 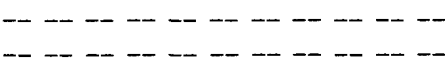 \\
\hline Paltechioceras (Orthechioceras?) sp & $\ldots+--\ldots+---$ & ---- & -- - - - - - - - - - - - - - - - \\
\hline Pseudolioceras $\mathrm{sp}$ & - - - - - - - - - - & ---- & -- - - - - - - - - - - - - - - - - \\
\hline $\begin{array}{l}\text { Haugia cf. H. grandis Buckman } \\
\text { H. cf. H. compressa Buckman }\end{array}$ & 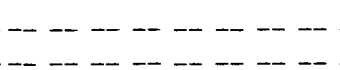 & & 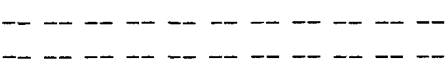 \\
\hline
\end{tabular}


area of the Kenai Peninsula, and in the Puale Bay-Alinchak Bay and Wide Bay areas of the Alaska Peninsula

Higher numbers are USGS Mesozoic locality numbers]

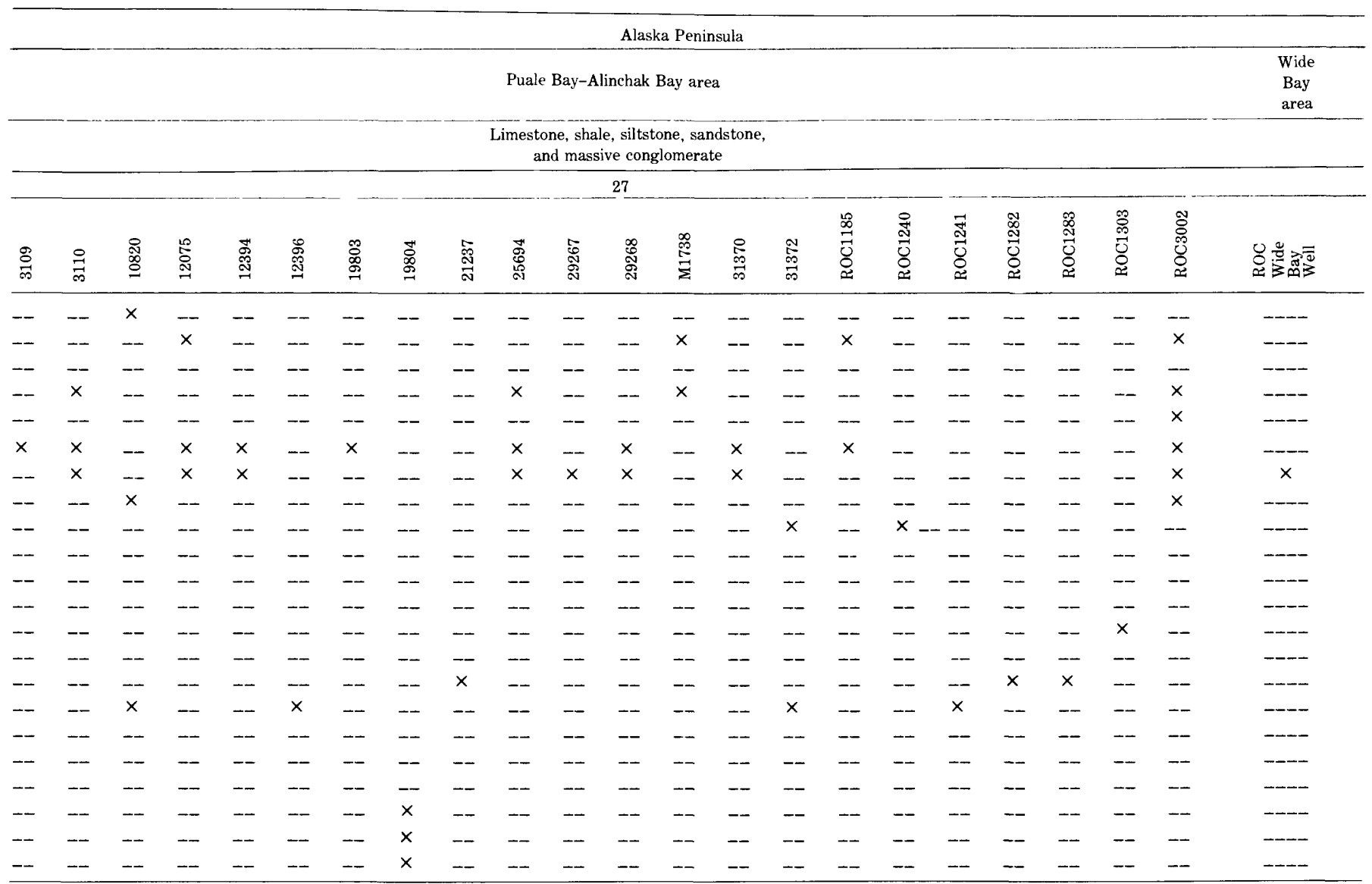


TABLE 7.-Description of Lower Jurassic fossil localities in Alaska

\begin{tabular}{cccc} 
& & [Leaders (_-_), number not assigned] \\
\hline $\begin{array}{c}\text { Locality No. } \\
\text { (figs. 2-6) }\end{array}$ & $\begin{array}{c}\text { USGS } \\
\text { Mesozoic } \\
\text { loc. No. }\end{array}$ & $\begin{array}{c}\text { Collector's } \\
\text { field No. }\end{array}$ & $\begin{array}{c}\text { Collector, year of collection, description of locality, } \\
\text { and stratigraphic assignment }\end{array}$ \\
\hline
\end{tabular}

(1 N Si

Simpson test well 1 , lat $70^{\circ} 57^{\prime} 05^{\prime \prime} \mathrm{N}$., long $155^{\circ} 21^{\prime}$

$45^{\prime \prime}$ W., Cape Simpson area, Barrow (A-2) quadrangle northern Alaska. Kingak Shale at depths of $5,677-5,691 \mathrm{ft}(1,730-1,735 \mathrm{~m})$. Late Pliensbachian.

Avak test well, lat $71^{\circ} 15^{\prime} 02^{\prime \prime} \mathrm{N}$., long $156^{\circ} 28^{\prime} 06^{\prime \prime}$ W., Point Barrow area, Barrow (B-4) quadrangle, northern Alaska. Kingak Shale at depths of $1,836 \mathrm{ft}(660 \mathrm{~m})$. Early Sinemurian.

South Barrow test well 3 , lat $71^{\circ} 15^{\prime} 51^{\prime \prime} \mathrm{N}$, long $156^{\circ} 37^{\prime} 55^{\prime \prime}$ W., Point Barrow area, Barrow (B-4) quadrangle, northern Alaska. Kingak Shale at depths of $1,772-2,470 \mathrm{ft}(540-753 \mathrm{~m})$. Early Sinemurian to early Toarcian.

South Barrow test well 12 , lat $71^{\circ} 14^{\prime} 18^{\prime \prime} \mathrm{N}$., long $156^{\circ} 20^{\prime} 06^{\prime \prime}$ W., Point Barrow area, Barrow (A-4) quadrangle, northern Alaska. Kingak Shale at depths of $1,987.4-2,181.5 \mathrm{ft}(606-665$ m). Hettangian to early Sinemurian.

British Petroleum (Alaska) Inc., 1964. On north bank of Thetis Creek headwaters, lat $68^{\circ} 41^{\prime} 51^{\prime \prime}$ N., long $164^{\circ} 45^{\prime} 05^{n} \mathrm{~W}$., north side of Delong Mountains, Delong Mts. (C-5) quadrangle, northern Alaska. Kingak Shale. Early to middle Toarcian.

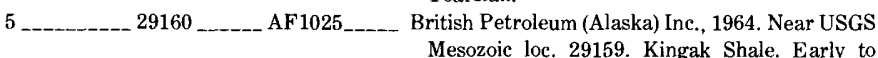
middle Toarcian.

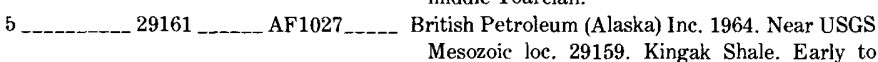
middle Toarcian.

5 _______________ British Petroleum (Alaska) Inc., 1964. Near USGS Mesozoic loc. 29159. Kingak Shale. Early to middle Toarcian.

5 _ 298776 ATo46F _- I. L. Tailleur and H. A. Tourtelot, 1968. Cutbank on large eastern tributary of Thetis Creek (near USGS Mesozoic loc. 29159 ), lat $68^{\circ} 40.8^{\prime} \mathrm{N}$., long $164^{\circ} 45.5^{\prime}$ W., Kingak Shale. Early to middle Toarcian.

6 ________ 29164 ______ AF26_____ British Petroleum (Alaska) Inc., 1964. North side of DeLong Mountains, on east bank of Ipewik River, lat $68^{\circ} 40^{\prime}$ N., long $164^{\circ} 13^{\prime}$ W., DeLong Mts. (C-4) quadrangle, northern Alaska. Unnamed beds. Late Pliensbachian.

6 _____ M2441____-_64ATr204F _ I. L. Tailleur, 1964. On Ipewik River on north side of Delong Mountains near USGS Mesozoic loc. 29164 , lat $68^{\circ} 37^{\prime}$ N., long $164^{\circ} 11^{\prime}$ W., northern Alaska. Unnamed beds. Late Pliensbachian.

7 ____ 29775 _____ 68ATo23____. I. Tailleur and H. A. Tourtelot, 1968. Cutbanks on east side of Ipnavik River, lat $68^{\circ} 40.6^{\prime} \mathrm{N}$., long $157^{\circ} 03.5^{\prime} \mathrm{W}$., Howard Pass quadrangle, northern Alaska. Unnamed oil shale overlying Shublik Formation (Triassic). Early Pliensbachian or latest Sinemurian.

$8 \quad 29774 \quad 68$ ATo21d I. L. Tailleur and H. A. Tourtelot, 1968. Blankenship Creek in Lisburne Ridge area, lat $68^{\circ} 37.5^{\prime}$ N., long $156^{\circ} 42.5^{\prime}$ W., Howard Pass quadrangle, northern Alaska. Unnamed oil shale overlying Triassic Shublik Formation. Early Pliensbachian.

9 ________ 29281 _____ 65ATr123B _ I. L. Tailleur, 1965. East wall of Tiglukpuk Creek on south flank of Tiglukpuk anticline, lat $68^{\circ} 21^{\prime} 15^{\prime \prime}$ N., long $151^{\circ} 5230^{\prime \prime}$ W., Chandler Lake quadrangle, northern Alaska. Unnamed beds overlying Shublik Formation. Early Pliensbachian or latest Sinemurian.

9 Creek, a tributary of Tiglukpuk Creek, just north of mountain front, lat $68^{\circ} 18^{\prime} 15^{\prime \prime} \mathrm{N}$., long $151^{\circ} 48^{\prime} 00^{\prime \prime}$ W., Chandler Lake quadrangle, northern Alaska. Float from unnamed beds overlying Shublik Formation. Early Pliensbachian or latest Sinemurian.

$10 \ldots 23772 \ldots$ 50AGr61__ R. W. Imlay, George Gryc, and Allan Kover, 1950. Near Red Hill on Ignek Creek, lat $69^{\circ} 36^{\prime}$
TABLE 7.-Description of Lower Jurassic fossil localities in Alaska-Continued

\begin{tabular}{cccc}
\hline $\begin{array}{c}\text { Locality No. } \\
\text { (figs. 2-6) }\end{array}$ & $\begin{array}{c}\text { USGS } \\
\text { Mesozoic } \\
\text { loc. No. }\end{array}$ & $\begin{array}{c}\text { Collector's } \\
\text { field No. }\end{array}$ & $\begin{array}{c}\text { Collector, year of collection, description of locality, } \\
\text { and stratigraphic assignment }\end{array}$ \\
\hline
\end{tabular}

N., long $146^{\circ} 06^{\prime}$ W., Mt. Michelson (C-4) quadrangle, northern Alaska. Kingak Shale. Late Toarcian.

11 _.____ 22081 _____ 48 ASa146____ E. G. Sable, 1948. About 1 mile (1.6 km) northeast of mouth of Fire Creek and $0.4 \mathrm{mi}(0.6 \mathrm{~km})$ above the mouth of a south-flowing tributary of the Sadlerochit River, Mt Michelson (C-2) quadrangle, northern Alaska. Kingak Shale, within middle third and about $1,700 \mathrm{ft}(518 \mathrm{~m})$ above its base in black earthy shale. Early to middle Toarcian.

12 _._______ 29165 ______ AG525_____ British Petroleum (Alaska) Inc., 1964. Three miles $(4.8 \mathrm{~km})$ east of Lake Schrader, lat $69^{\circ} 22^{\prime} \mathrm{N}$., long $144^{\circ} 28^{\prime}$ W., Mt. Michelson (B-2) quadrangle, northern Alaska. Kingak Shale. Late Pliensbachian.

13 71ADt330c 30074 R. L. Detterman, 1971. Cutbank on west side of Aichilik River about $2 \mathrm{mi}(3.2 \mathrm{~km}) \mathrm{N} .30^{\circ} \mathrm{E}$. of VABM ATTE on northern front of Brooks Range, lat $69^{\circ} 33^{\prime}$ N., long $143^{\circ} 05^{\prime}$ W., Barter Island (C-4) quadrangle, northern Alaska. Kingak Shale, 800 to $1,000 \mathrm{ft}$ ( 244 to $205 \mathrm{~m}$ ) above base. Late Pliensbachian.

14 _ 29737 ___-_ 63ABe47B _._ W. P. Brosgé, 1963. Spike Mountain, about 3 miles $(48 \mathrm{~km})$ north of Old Rampart, lat $63^{\circ} 35.75^{\prime} \mathrm{N}$., long $141^{\circ} 40^{\prime} \mathrm{W}$., Porcupine River area, Coleen quadrangle, east-central Alaska. Glenn Shale (in part). A thin unit of red conglomeratic sandstone interbedded with black fine-grained quartzitic sandstone. Early Hettangian.

14 _-_ 29738 _-_-_ 67ARr-337a_ W. P. Brosgé, 1967. Same location as USGS Mesozoic loc. 29737. Fossils from apparent top of $120 \mathrm{ft}(36.6 \mathrm{~m})$ of beds overlying granite. Ear ly Hettangian.

14 _____ 29739 _ $67 \mathrm{ARr}-337 \mathrm{~d}_{\text {_ }}$ Same data as Mesozoic loc. 29738 but from $20 \mathrm{ft}$ $(6 \mathrm{~m})$ below apparent top. Early Hettangian.

14 _______ 29740 _.___ 67ARr3371-n Same data as Mesozoic loc. 29738 but from $40 \mathrm{ft}$ $14 \quad 67 \mathrm{ARr}-\quad \begin{array}{r}(12 \mathrm{~m}) \text { below apparent top. Early Hettangian. } \\ \text { Same data as Mesozoic loc. } 29738 \text { but from } 80 \mathrm{ft}\end{array}$

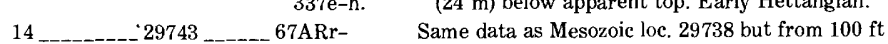
$(30.5 \mathrm{~m})$ below apparent top. Early Hettangian. British Petroleum (Alaska) Inc. Float from river bluff in the middle fork Salmon Trout River about 9 miles $(14.4 \mathrm{~km})$ east southeast of Old Rampart in Porcupine River area, Coleen quadrangle, east-central Alaska. Glenn Shale (in part). Noncalcareous, pyritic, thin-bedded mudstone interbedded with dark-gray papery shale exposed on bluff. Late Pliensbachian.

16 _____ 29773 _____ 68 s54F __ George Plafker, 1968. Float from sec. 34, T. 23 S.,

R. 35 E., Yakutat (D-4) quadrangle, southeastern Alaska. Yakutat Group. Probably early Pliensbachian rather than late Sinemurian.

$1728671 \quad 62 \mathrm{AMK}-66$ E. M. MacKevett, Jr. 1962 . On ridge in southcentral part of sec. 23 , T. 4 S., R. 16 E., McCarthy (C-4) quadrangle, Wrangell Mountains, southern Alaska. Upper member of McCarthy Formation. Less than $100 \mathrm{ft}(30.5 \mathrm{~m})$ stratigraphically below the Lubbe Creek Formation. Early Pliensbachian.

17 _ 28672 ___ 62 AMK-66A E. M. MacKevett, Jr., 1962. Same description as Mesozoic loc. 28671 and same age.

17 __-___-_ 28673 __-_-_ 62 AMK-67__ E. M. MacKevett, Jr., 1962. Collected slightly east of Mesozoic loc. 28671 at the same stratigraphic position.

17 62AMK-69 E. M Mackevett, Jr. 1962. West-central part of NW1/4 SW1/4 sec. 23 , T. 4 S., R. 16 E., at altitude of $6,300 \mathrm{ft}(1,920 \mathrm{~m})$, McCarthy $(\mathrm{C}-4)$ quadrangle, Wrangell Mountains, southern Alaska. Upper member of McCarthy Formation, less than $100 \mathrm{ft}(30.5 \mathrm{~m})$ stratigraphically below Lubbe Creek Formation. Early Pliensbachian. 
TABLE 7.-Description of Lower Jurassic fossil localities in Alaska-Continued

\begin{tabular}{cccc}
\hline $\begin{array}{c}\text { Locality No. } \\
\text { (figs. 2-6) }\end{array}$ & $\begin{array}{c}\text { USGS } \\
\text { Mesozoic } \\
\text { loc. No. }\end{array}$ & $\begin{array}{c}\text { Collector's } \\
\text { field No. }\end{array}$ & $\begin{array}{c}\text { Collector, year of collection, description of locality, } \\
\text { and stratigraphic assignment }\end{array}$ \\
\hline
\end{tabular}$$
17
$$

28677 $62 \mathrm{AMK}-88$ sec. 24 , T. 4 S., R. 16 E., McCarthy (C-4) quadrangle, Wrangell Mountains, southern Alaska. Upper member of McCarthy Formation. Float from impure limestone near an intraformational fault. Early Sinemurian.

$17 \ldots 29870 \ldots$ ___ 67 AWK-112_ E. M. MacKevett, Jr., 1967. On north side of Chitistone Mountain in east-central part of NE $1 / 4 N^{1 / 4}$ sec. 27, T. 4 S., R. 16 E., McCarthy (B-4) quadrangle, Wrangell Mountains, southern Alaska. Upper member(?) of McCarthy Formation. Hettangian. $17 \ldots 29890 \quad 67$ AWK-113_ E. M. MacKevett, Jr., 1967, near Mesozoic loc.
29870 in same quarter of a quarter section and same stratigraphic position. Hettangian.

17 _ 29891 67AMK-233_ E. M. MacKevett, Jr., 1967. NE1/4 NW1/4 sec. 26, T. 4 S., R. 16 E., McCarthy (B-4) quadrangle, Wrangell Mountains. Upper member of McCarthy Formation, a few hundred feet $(100 \mathrm{~m})$ above base of member. Hettangian.

18 27AM-F5 _ F. H. Moffit, 1927. McCarthy Creek valley, near creek on east side, $2.5 \mathrm{mi}(4 \mathrm{~km})$ from the glacier, NE $1 / 4 \mathrm{NE}^{1 / 4} \mathrm{sec}$. 36 , T. 3 S., R. 14 E., McCarthy (C-5) quadrangle, Wrangell Mountains, southern Alaska. Upper member of McCarthy Formation, probably a few hundred feet $(100 \mathrm{~m})$ above base. Late Sinemurian.

18 ________ $28531 \ldots$ _ $19946-39 \ldots$ Standard Oil Co. of California. East of MeCarthy Creek near center sec. 25, T. $3 \mathrm{~S}$., R, $14 \mathrm{E}$, McCarthy (C-5) quadrangle, Wrangell Mountains, southern Alaska. Lubbe Creek Formation. Late Pliensbachian.

$18 \quad 28533 \quad$ F-6 2 E. M. MacKevett, Jr., 1961. SE $18 \mathrm{NE}^{1 / 4} \mathrm{NW}^{1 / 4} \mathrm{sec}$ 2, T. 4 S., R. 14 E., McCarthy (C-5) quadrangle, Wrangell Mountains, southern Alaska. Upper member of McCarthy Formation. About $1,000 \mathrm{ft}$ $(305 \mathrm{~m})$ stratigraphically above base of member. Early Sinemurian.

18 _____ 28534 E10_. M. MacKevett, Jr., 1961. On ridge west of McCarthy Creek, SW1/4 NW1/4 NW1/4 sec. 26, T. 3 S., R. 14 E., McCarthy (C-5) quadrangle, Wrangell Mountains, southern Alaska. Upper member of McCarthy Formation, a few feet (about $1 \mathrm{~m}$ ) below upper contact. Early Pliensbachian.

$18 \ldots 28535 \ldots$ F _ E. M. MacKevett, Jr., 1961. South-central part $\mathrm{NW}^{1 / 4} \mathrm{SE}^{1 / 4} \mathrm{sec}$. 11, T. 4. S., R. 15 E., McCarthy (C-5) quadrangle, Wrangell Mountains, southern Alaska. Float from upper member of McCarthy Formation. Early Sinemurian.

18 _______ 28537 ____ F36_____._ MacKevett, Jr., 1961. NE. cor. sec. 16., T. 4 S., R. 15 E., McCarthy (C-5) quadrangle, Wrangell Mountains, southern Alaska. Upper member of McCarthy Formation; probably in middle part of member. Early Sinemurian.

18 _______ E. M. MacKevett, Jr., 1961. A little south of USGS Mesozoic loc. 28537. Early Sinemurian.

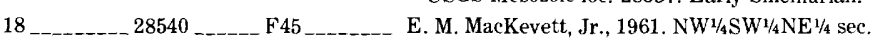
9. T. 4 S., R. 15 E., McCarthy (C-5) quadrangle, Wrangell Mountains, southern Alaska. Float collected about $400 \mathrm{ft}(122 \mathrm{~m})$ below top of upper member of McCarthy Formation. Early Pliensbachian.

$18 \quad 28678 \quad 62 \mathrm{AMK}-20 \quad$ R. W. Imlay, 1962. Southwest of West Fork in NE. cor. NW1/4 sec. 3 , T. 4 S., R. 15 E., McCar thy (C-5) quadrangle, Wrangell Mountains, southern Alaska. Lubbe Creek Formation. Early Toarcian(?)

18 _____ 28688 _.___ 62AMK-143_ E. M. MacKevett, Jr., 1962 West of Nizina River in $\mathrm{SE}^{1 / 4} \mathrm{NW}^{1 / 4} \mathrm{SW}^{1 / 4}$ sec. 16, T. 3 S. R. $16 \mathrm{E}$., Mc Carthy $(\mathrm{C}-5)$ quadrangle, Wrangell Mountains, southern Alaska. Near top of upper member of McCarthy Formation. Late Pliensbachian.

18 _._-_ 28690 _ $62 \mathrm{AMK}-16$ _- R. W. Imlay, 1962. In saddle on ridge between
TABLE 7.-Description of Lower Jurassic fossil localities in Alaska-Continued

\begin{tabular}{|c|c|c|c|}
\hline $\begin{array}{l}\text { Locality No. } \\
\text { (figs. 2-6) }\end{array}$ & $\begin{array}{l}\text { USGS } \\
\text { Mesozoic } \\
\text { loc. No. }\end{array}$ & $\begin{array}{l}\text { Collector's } \\
\text { field No. }\end{array}$ & $\begin{array}{l}\text { Collector, year of collection, description of locality, } \\
\text { and stratigraphic assignment }\end{array}$ \\
\hline & & & $\begin{array}{l}\text { Lubbe Creek and Diamond Creek east of McCar- } \\
\text { thy Creek, in east-central part of NE } 1 / 4 \text { sec. } 7, \mathrm{~T} \text {. } \\
4 \mathrm{~S} \text {., R. } 15 \mathrm{E} \text {., McCarthy (C-5) quadrangle, } \\
\text { Wrangell Mountains, southern Alaska. Upper } \\
\text { member of McCarthy Formation. Probably late } \\
\text { Sinemurian. }\end{array}$ \\
\hline
\end{tabular}

18 ___ 28704 __AMK-62A E. M. MacKevett, Jr., 1962. West of McCarthy Creek opposite Lubbe Creek on ridge in eastcentral part of SW1/4 sec. 35, T. 3 S., R. 14 E. McCarthy (C-5) quadrangle, Wrangell Mountains, southern Alaska. About 1,200 ft (366 m) above base of upper member of McCarthy Formation. Probably late Sinemurian.

$18 \ldots 28706 \ldots$ ___ 62 AMK-63__ E. M. MacKevett, Jr., 1962. On ridge west of MeCarthy Creek near center $\mathrm{NW}^{1 / 4 \mathrm{NW}^{1 / 4}}$ sec. 35, T. 3 S., R. 14 E., McCarthy (C-5) quadrangle, Wrangell Mountains, southern Alaska. In upper part of upper member of McCarthy Formation. Early Sinemurian.

18 ____ 29118 64AMK-82__ E. M. MacKevett, Jr., 1964. South-central part of SW $^{1 / 4} \mathrm{NW}^{1 / 4} \mathrm{sec}$ s5, T. 4 S., R. 15 E., McCarthy (B-5) quadrangle, Wrangell Mountains, southern Alaska. Transitional beds at base of upper member of McCarthy Formation. Early Sinemurian.

18 ______ 29119 ____ 64 AMK-84__ E. M. MacKevett, Jr., $1964.300 \mathrm{ft}(91.5 \mathrm{~m})$ southeast of USGS Mesozoic loc. 29118 in same quarter of a quarter section. Transitional beds at base of upper member of McCarthy Formation. Early Sinemurian.

18 ____ 29121 _ 64 AMK-108_ E. M. MacKevett, Jr., 1964. In area of small folds $40 \mathrm{ft}(12 \mathrm{~m}) \mathrm{S} .60^{\circ} \mathrm{W}$. of summit of Hill 6580 . $\mathrm{NE}^{1 / 4 \mathrm{NE}^{1 / 4}}$ sec. 34 , T. 4 S., R. 15 E., McCarthy (B-5) quadrangle, Wrangell Mountains, southern Alaska. Low in upper member of McCarthy Formation. Early Sinemurian.

18 _____ 31174 ___ $63 \mathrm{AWz}-150 \_$E. M. MacKevett, Jr., 1963. On ridge between Root Glacier and Kennicott Glacier. NE1/4 $\mathrm{SW}^{1 / 4}$ sec. 19, T. 3 S., R. 14 E., McCarthy (C-16) quadrangle, Wrangell Mountains, southern Alaska. Upper member of McCarthy Formation, $150 \mathrm{ft}(46 \mathrm{~m})$ below top. Late Sinemurian.

18 ___ 31175 _ $63 \mathrm{AWz}-152$ _ E. M. MacKevett, Jr., 1963. Same location as USGS Mesozoic loc. 31174 but only $100 \mathrm{ft}(30.5$ $\mathrm{m})$ below top of upper member of McCarthy Formation. Late Sinemurian.

19 28AM-F9 _ F. H. Moffit, 1928. Head of first northern tributary of Mill Creek, SE1/4 sec. 33, T. 3 S., R. 11 E., McCarthy (C-7) quadrangle, Wrangell Mountains, southern Alaska. Upper member of McCarthy Formation. Late Sinemurian.

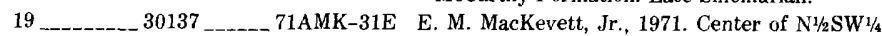
sec. 8 , T. 4 S., R. 11 E., McCarthy (C-7) quadrangle, Wrangell Mountains, southern Alaska. Lower part of upper member of McCarthy Formation. Early Sinemurian.

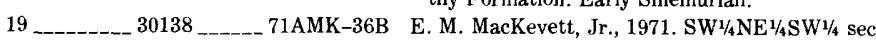
17, T. 4 S., R. 12 E., McCarthy (C-7) quadrangle, Wrangell Mountains, southern Alaska. Low in upper member of McCarthy Formation. Early Sinemurian.

19 of $\mathrm{SE}^{1 / 4} \mathrm{NW}^{1 / 4} \mathrm{sec} .17$, T. $4 \mathrm{~S}$., R. $12 \mathrm{E}$., McCarthy (C-7) quadrangle, Wrangell Mountains, southern Alaska. Low in upper member of McCarthy Formation. Late Sinemurian.

$19 \quad 30140 \quad 71 \mathrm{AFd} 264 \quad$ A. B. Ford, 1971. On ridge in SW cor sec 17 near sec. 18, T. 4 S., R. 12 E., McCarthy (C-7) quadrangle, Wrangell Mountains, southern Alaska. Early Sinemurian.

$20 \quad 25939 \quad 55 A G z 182$ Arthur Grantz, 1955. Lat $61^{\circ} 54^{\prime} 02^{\prime \prime}$ N., long $146^{\circ} 52^{\prime} 00^{\prime \prime}$ W., Valdez (D-8) quadrangle, south side of western Copper River basin, southern Alaska. Talkeetna Formation. Late Toarcian. 
TABLE 7.-Description of Lower Jurassic fossil localities in Alaska-Continued

\begin{tabular}{|c|c|c|c|}
\hline $\begin{array}{l}\text { Lorality No. } \\
\text { (figs. 2-6) }\end{array}$ & $\begin{array}{c}\text { USGS } \\
\text { Mesozoic } \\
\text { loc. No. }\end{array}$ & $\begin{array}{l}\text { Collector's } \\
\text { field No. }\end{array}$ & $\begin{array}{l}\text { Collector, year of collection, description of locality, } \\
\text { and stratigraphic assignment }\end{array}$ \\
\hline 21 & 4107 & $52 \mathrm{AGz} 63_{-}$ & $\begin{array}{l}\text { R. D. Hoare, R. W. Imlay, and Arthur Grantz, } \\
\text { 1952. On south tributary of Squaw Creek, } 1.98 \\
\text { miles }(3.2 \mathrm{~km}) \mathrm{N} .28^{1 / 1} 2^{\circ} \text { E. of southeast summit } \\
\text { of Gunsight Mountain, Anchorage (D-2) quad. } \\
\text { Talkeetna Mountains, southern Alaska. Talkeet- } \\
\text { na Formation. Late Pliensbachian. }\end{array}$ \\
\hline 21 & 4108 & $52 \mathrm{AGz66}$ & $\begin{array}{l}\text { Arthur Grantz, R. D. Hoare, and R. W. Imlay, } \\
\text { 1952. Differs from USGS Mesozoic loc. } 24107 \text { by } \\
\text { being } 1.91 \text { miles }(3.1 \mathrm{~km}) \text { N. } 28^{1 / 2}{ }^{\circ} \text { E. of } \\
\text { southeast summit of Gunsight Mountain. Late } \\
\text { Pliensbachian. }\end{array}$ \\
\hline 21 & 4111 & $52 \mathrm{AGz} 253$ & $\begin{array}{l}\text { Arthur Grantz, R. D. Hoare, and R. W. Imlay, } \\
\text { 1952. } 5.04 \text { miles }(8 \mathrm{kms}) \text { N. } 33^{\circ} \text { E. of the mouth } \\
\text { of Sheep Creek, Anchorage (D-2) quadrangle, } \\
\text { Talkeetna Mountains, southern Alaska. Early } \\
\text { Toarcian. }\end{array}$ \\
\hline 21 & 4114 & $52 \mathrm{AGz} 98$ & $\begin{array}{l}\text { Arthur Grantz, R. D. Hoard, and R. W. Imlay, } \\
\text { 1952. From north branch of North Creek, } 1.61 \\
\text { miles }(2.5 \mathrm{~km}) \mathrm{N} .45^{\circ} \text { W. of its mouth. Talkeetna } \\
\text { Mountains (A-1) quadrangle, southern Alaska. } \\
\text { Talkeetna Formation. Late Toarcian. }\end{array}$ \\
\hline 21 & 4787 & $53 \mathrm{AGz40}$ & $\begin{array}{l}\text { Arthur Grantz and L. F. Fay, } 1953 \text {. About } 0.6 \text { mile } \\
(1 \mathrm{~km}) \text { northwest of point where Camp Creek } \\
\text { crosses Glenn Highway, lat } 61^{\circ} 50^{\prime} 53^{\prime \prime} \text { N., long } \\
147^{\circ} 24^{\prime} 43^{\prime \prime} \text { W., Anchorage (D-2) quadrangle, } \\
\text { Talkeetna Mountains, southern Alaska. Talkeet- } \\
\text { na Formation. Early Toarcian. }\end{array}$ \\
\hline 21 & 5316 & $54 \mathrm{AGz48 \textrm {A }}$ & $\begin{array}{l}\text { Arthur Grantz and L. F. Fay, } 1954 \text {. Lat } 62^{\circ} 00^{\prime} 42^{\prime \prime} \\
\text { N., long } 147^{\circ} 27^{\prime} 49^{\prime \prime} \text { W., Talkeetna Mountains } \\
\text { (A-1) quadrangle, Talkeetna Mountains, } \\
\text { southern Alaska. Talkeetna Formation, upper } \\
\text { part. Late Toarcian. }\end{array}$ \\
\hline $21 \ldots$ & 5317 & $54 \mathrm{AGz} 49 \mathrm{~A}$ & $\begin{array}{l}\text { Arthur Grantz and L. F. Fay, } 1954 \text {. Lat } 62^{\circ} 00^{\prime} 06^{\prime \prime} \\
\text { N., long } 147^{\circ} 31^{\prime} 30^{\prime \prime} \text { W., Talkeetna Mountains } \\
\text { (A-2) quadrangle, Talkeetna Mountains, } \\
\text { southern Alaska. Upper part of Talkeetna For- } \\
\text { mation. Late Toarcian. }\end{array}$ \\
\hline $21 \ldots$ & 5318 & $54 \mathrm{AGz50}$ & $\begin{array}{l}\text { Arthur Grantz and L. F. Fay, } 1954 \text {. Lat } 62^{\circ} 00^{\prime} 10^{\prime \prime} \\
\text { N., long } 147^{\circ} 32^{\prime} 33^{\prime \prime} \text { W., Talkeetna Mountains } \\
\text { (A-2) quadrangle, Talkeetna Mountains, } \\
\text { southern Alaska. Talkeetna Formation. Late } \\
\text { Toarcian. }\end{array}$ \\
\hline 21 & 5319 & $54 \mathrm{AGz51}$ & $\begin{array}{l}\text { Arthur Grantz and L. F. Fay, 1954. Lat } 62^{\circ} 00^{\prime} 00 \\
1^{\prime \prime 2} \text { N., long } 147^{\circ} 32^{\prime} 45^{\prime \prime} \text { W., Talkeetna Mountains } \\
\text { (A-2) quadrangle, Talkeetna Mountains, } \\
\text { southern Alaska. Talkeetna Formation. Late } \\
\text { Toarcian. }\end{array}$ \\
\hline 21 & 25342 & $04 \pi x y<4$ & $\begin{array}{l}\text { L. F. Fay, } 1954 \text {. Lat } 62^{\circ} 02^{\prime} 48^{\prime \prime} \text { N., long } \\
147^{\circ} 29^{\prime \prime} 34^{\prime \prime} \text { W., Talkeetna (A-1) quadrangle, } \\
\text { Talkeetna Mountains, southern Alaska. Talkeet- } \\
\text { na Formation. Late Toarcian. }\end{array}$ \\
\hline 21 & 5359 & $54 \mathrm{AFy} 130$ & $\begin{array}{l}\text { L. F. Fay, 1954. Lat } 62^{\circ} 02^{\prime} 40^{\prime \prime} \text { N., long } \\
147^{\circ} 21^{\prime} 36^{\prime \prime} \text { W., Talkeetna Mountains (A-1) } \\
\text { quadrangle, Talkeetna Mountains, southern } \\
\text { Alaska. Talkeetna Formation. Late Toarcian. }\end{array}$ \\
\hline 21 & 5940 & $55 \mathrm{AGz} 228 \mathrm{a}_{-}$ & $\begin{array}{l}\text { Arthur Grantz, } 1955 \text {. Lat } 62^{\circ} 01^{\prime} 37^{\prime \prime} \text { N., long } \\
147^{\circ} 17^{\prime} 53^{\prime \prime} \text { W., Talkeetna Mountains (A-1) } \\
\text { quadrangle, Talkeetna Mountains, southern } \\
\text { Alaska. Talkeetna Formation. Late Toarcian. }\end{array}$ \\
\hline $21 \ldots$ & 5941 & $55 \mathrm{AGz} 294$ & $\begin{array}{l}\text { Arthur Grantz, } 1955 \text {. Lat } 61^{\circ} 51^{\prime} 51^{\prime \prime} \text { N., long } \\
147^{\circ} 21^{\prime} 22^{\prime \prime} \text { W., at east end of Sheep Mountain. } \\
\text { Anchorage (D-1) quadrangle, Talkeetna Moun- } \\
\text { tains, southern Alaska. Talkeetna Formation, } \\
\text { upper part. Late Pliensbachian. }\end{array}$ \\
\hline 21 & 7508 & $6-10 \mathrm{BB}_{-}$ & $\begin{array}{l}\text { General Petroleum Corp. 1959. Near head of } \\
\text { McDougall Creek. Coordinates 15.9-0.37. } \\
\text { Talkeetna Mountains (A-2) quadrangle, } \\
\text { Talkeetna Mountains, southern Alaska. Talkeet- } \\
\text { na Formation. Late Toarcian. }\end{array}$ \\
\hline 21 & 449 & & $\begin{array}{l}\text { Arthur Grantz, 1966. Same place and strati- } \\
\text { graphic position as USGS Mesozoic loc. } 24108 .\end{array}$ \\
\hline 21 & 9450 & $66 \mathrm{AGz} 31 \mathrm{~B}$ & $\begin{array}{l}\text { Arthur Grantz, 1966. On south tributary of Squaw } \\
\text { Creek, } 1.95 \text { miles }(3.1 \mathrm{~km} \text { ) southeast of summit } \\
\text { of Gunsight Mountain, near USGS Mesozoic loc. } \\
\text { 24107. Anchorage (D-2) quadrangle, Talkeetna }\end{array}$ \\
\hline
\end{tabular}

TABLE 7.-Description of Lower Jurassic fossil localities in Alask $a-$ Continued

\begin{tabular}{cccc} 
Locality No. & USGS \\
(figs. 2-6) & $\begin{array}{c}\text { Mesozoic } \\
\text { loc. No. }\end{array}$ & $\begin{array}{c}\text { Collector's } \\
\text { field No. }\end{array}$ & $\begin{array}{c}\text { Collector, year of collection, description of locality, } \\
\text { and stratigraphic assignment }\end{array}$ \\
\hline
\end{tabular}

\begin{tabular}{llll} 
(figs. 2-6) & loc. No. & field No. & and stratigraphic assignment \\
\hline & & Mountains, southern Alaska. Talkeetna Forma-
\end{tabular}
tion. Late Pliensbachian.

22_________ M6171______ 73APr85A __. George Plafker, 1973. On south-flowing branch of Doone Creek, $2,000 \mathrm{ft}(610 \mathrm{~m})$ west of SE. cor. sec. 25, T. 21 N., R. 5 E., Anchorage (D-4) quadrangle, Talkeetna Mountains, southern Alaska. Talkeetna Formation. Early Pliensbachian.

22 ___ Aug. 30,1910 A. H. Brooks, 1910. Creek entering Chickaloon River from west 1 mile $(1.6 \mathrm{~km})$ above Government Bridge at altitude of $2,000 \mathrm{ft}(610 \mathrm{~m})$. Coordinates $3.0-9.0$. Anchorage D-4 quadrangle, Talkeetna Mountains, southern Alaska. Talkeetna Formation. Late Sinemurian.

$22 \quad 6797 \quad$ Aug. 30.1910 G. C. Martin, 1910. Doone Creek at mouth of gulch. Anchorage (D-4) quadrangle, Talkeetna Mountains, southern Alaska. Float from Talkeetna Formation. Early Pliensbachian.

22 27586 13177-2 Standard Oil of California. Carbon Creek, 2.3 miles $(3.7 \mathrm{~km}) \mathrm{S} .7^{\circ} \mathrm{E}$. of its mouth near center of $\mathrm{SE}^{1 / 4}$ sec. 12 , T. 19 N., R. 5 E., Anchorage (C-4) quadrangle, coordinates 1.4-17.3., Chugach Mountains, southern Alaska. Talkeetna Formation. Late Pliensbachian.

22 _________ 29198 ______ SUS62 ____- British Petroleum Expl. (Alaska) Co. From saddle on south side of Kings Mountain $21 / 2$ miles (4 $\mathrm{km}) \mathrm{S} .15^{\circ} \mathrm{E}$. of VABM 757 on the Glenn Highway. Anchorage (C-5) quadrangle. Chugach Mountains, southern Alaska. Talkeetna Formation. Early Toarcian.

$23 \quad 57 \mathrm{AGz30} \quad$ Arthur Grantz, 1957. Lat $62^{\circ} 59^{\prime} 58^{\prime \prime}$ N., long $147^{\circ} 57^{\prime} 48^{\prime \prime}$ W., Coordinates 1.20-17.23. Talkeetna Mountains (A-2) quadrangle, Talkeetna Mountains, southern Alaska. Talkeetna Formation. Late Sinemurian.

$23 \quad 28661 \quad 62 \mathrm{AGz} 84$ Arthur Grantz, 1962, on Nowhere Creek, Lat $62^{\circ} 13^{\prime} 29^{\prime \prime}$ N., long $148^{\circ} 01^{\prime} 27^{\prime \prime}$ W., Coordinates 15.35-15.63. Talkeetna Mountains (A-3) quadrangle, Talkeetna Mountains, southern Alaska. Talkeetna Formation. Late Sinemurian.

23 ___-_ 28662 __-_ 62AGz84A _-_ Arthur Grantz, 1962. On Nowhere Creek, lat $62^{\circ} 13^{\prime} 30^{\prime \prime}$ N., long $148^{\circ} 01^{\prime} 18^{\prime \prime}$ W., Coordinates 15.43-15.65., Talkeetna Mountains (A-3) quadrangle, Talkeetna Mountains, southern Alaska. Late Sinemurian.

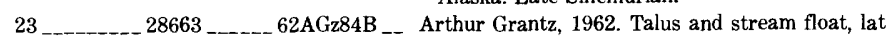
$62^{\circ} 13 \prime 30^{\prime \prime}$ N., long $148^{\circ} 0^{\prime} 12^{\prime \prime}$ W., Coordinates 15.5-15.65., Talkeetna Mountains (A-3) quadrangle, Talkeetna Mountains, southern Alaska. Talkeetna Formation. Late Sinemurian.

24 _____________ Elmer Bedager, 1932. Head of an eastern tributary of Partin Creek that heads against Little Shotgun Creek on west side of Chulitna River valley north of Eldridge Glacier. Probably same place as USGS Mesozoic loc. 31262 in Healy (A-6) quadrangle, south-central part of Alaska Range, southern Alaska. Unnamed beds. Late early to early middle Sinemurian.

24 ___ 31260 _____ F6-J-27 ____ N. J. Silberling and D. L. Jones, 1976. On divide between Ohio and Copeland Creeks; $5,800 \mathrm{ft}$ $(1,768 \mathrm{~m}) \mathrm{N} .30^{\circ} \mathrm{E}$. of VABM Copeland, Healy (A-6) quadrangle, south-central part of Alaska Range, southern Alaska. Unnamed beds. Late early to early middle Sineumurian.

$24 \ldots 31261 \ldots$ F6-J-31___ N. J. Silberling and D. L. Jones, 1976. On divide between Ohio and Copeland Creek; $6,800 \mathrm{ft}$ $(2073 \mathrm{~m}) \mathrm{N} .39^{\circ} \mathrm{E}$. from VABM Copeland, Healy (A-6) quadrangle, south-central part of Alaska Range, southern Alaska. Unnamed beds. Latest Sinemurian.

$24 \ldots 31262 \ldots$ F6-S-322___ N. J. Silberling and D. L. Jones, 1976. Divide between Partin and Little Shotgun Creeks in upper part of small drainage into Partin Creek, $39,850 \mathrm{ft}(12,147 \mathrm{~m}) \mathrm{S} .31 .5^{\circ} \mathrm{W}$. from VABM Copeland, Healy (A-6) quadrangle, southcentral part of Alaska Range, southern Alaska. 
TABLE 7.-Description of Lower Jurassic fossil localities in Alaska-Continued

$\begin{array}{lccc}\begin{array}{c}\text { Locality No. } \\ \text { (figs. 2-6) }\end{array} & \begin{array}{c}\text { USGS } \\ \text { Mesozoic } \\ \text { loc. No. }\end{array} & \begin{array}{c}\text { Collector's } \\ \text { field No. }\end{array} & \begin{array}{c}\text { Collector, year of collection, description of locality, } \\ \text { and stratigraphic assignment }\end{array} \\ \text { Unnamed calcareous sandstone. Late early to }\end{array}$
early middle Sinemurian. between Ohio and Shotgun Creeks $19,800 \mathrm{ft}$ $(6,035 \mathrm{~m}) \mathrm{S} .38^{\circ} \mathrm{W}$. from VABM Copeland, Healy (A-6) quadrangle, south-central part of Alaska Range, southern Alaska. Unnamed beds. Late early to early middle Sinemurian.

24 _____ $31264 \ldots$ F6-S331___ N. J. Silberling and D. L. Jones, 1976. Divide between Shotgun and Little Shotgun Creeks, $28,800 \mathrm{ft}(8778 \mathrm{~m}) \mathrm{S} .34^{\circ} \mathrm{W}$. from VABM Copeland, Healy (A-6) quadrangle, southcentral part of Alaska Range, southern Alaska. Unnamed beds. Earliest Sinemurian.

$2431265 \quad$ F6-S-332 _ N. J. Silberling and D. L. Jones, 1976. Divide between Shotgun and Little Shotgun Creeks, $31,000 \mathrm{ft}(9,449 \mathrm{~m}) \mathrm{S}$. $33^{\circ} \mathrm{W}$. from VABM Copeland, Healy (A-6) quadrangle, southcentral part of Alaska Range, southern Alaska. Unnamed beds. Late early to early middle Sinemurian.

24 _ _ 31266 _____ F6-S-333___ N. J. Silberling and D. L. Jones, 1976. Crest of ridge on east side of upper Long Creek, 32,700 ft $(9,967 \mathrm{~m}) \mathrm{N}$. $6^{\circ}$ E. from VABM Copeland, Healy (A-6) quadrangle, south-central part of Alaska Range, southern Alaska. Unnamed beds. Earliest Sinemurian.

25 __ 30907 _ 75 AR38A _-_ R. L. Detterman and D. L. Jones, 1975. Altitude $5,850 \mathrm{ft}(1,768 \mathrm{~m}), 3.5 \mathrm{mi}(5.6 \mathrm{~km})$ west of Shellabarger Pass, sec. 12, T. 28 N., R. 20 W., lat $62^{\circ} 31^{\prime} 49^{\prime \prime} \mathrm{N}$., long $152^{\circ} 53^{\prime} 02^{\prime \prime} \mathrm{W}$., Talkeetna (C-6) quadrangle, south-central part of Alaska Range, southern Alaska. Unnamed beds $150 \mathrm{ft}$ $(45.7 \mathrm{~m})$ below lowest $B u c h i a$-bearing limestone. Sinemurian.

$2530908 \ldots 75$ AR39 ___ D. L. Jones, 1975 . Altitude $6,100 \mathrm{ft}(1,859 \mathrm{~m})$, 5 miles $(8 \mathrm{~km})$ southwest of Shellabarger Pass, lat $62^{\circ} 30^{\prime} 20^{\prime \prime} \mathrm{N}$., long $152^{\circ} 55^{\prime} 59^{\prime \prime} \mathrm{W}$., Talkeetna (C-6) quadrangle, south-central part of Alaska Range, southern Alaska. Unnamed beds. Early Sinemurian.

$25 \ldots 31270 \ldots$ (76 AR17)___ Bruce Reed and R. L. Detterman, 1976. Float from 100 to $150 \mathrm{ft}$ ( 30 to $46 \mathrm{~m}$ ) of siltstone and shale exposed on north side of ridge about 1 mile $(1.6 \mathrm{~km})$ northwest of Tatina River in southcentral part of $\mathrm{SW}^{1 / 4} \mathrm{SEE}^{1 / 4}$ sec. 25 , T. $28 \mathrm{~N}$., R. $20 \mathrm{~W}$., lat $62^{\circ} 28^{\prime} 51^{\prime \prime}$ N., long $152^{\circ} 53^{\prime \prime} 36^{\prime \prime}$ W., Talkeetna (B-6) quadrangle, south-central part of Alaska Range, southern Alaska. Early Sinemurian.

26 ___ 2978 T. W. Stanton and G. C. Martin, 1904. Point Naskowhak, at entrance to Seldovia Bay, Seldovia (B-5) quadrangle, on Kenai Peninsula, southern Alaska. Unnamed beds. Early Sinemurian.

262979 T. W. Stanton and G. C. Martin, 1904. Threefourths of a mile $(1.2 \mathrm{~km})$ west of entrance to Seldovia Bay, Seldovia (B-5) quadrangle on Kenai Peninsula, southern Alaska. Unnamed beds. Early Sinemurian.

$26 \quad 2981 \quad 907 \mathrm{~b}$ miles $(3.2 \mathrm{~km})$ west of Seldovia Bay, eastern part of sec. 34, T. 8 S., R. 15 W., Seldovia (B-5) quadrangle, on Kenai Peninsula, southern Alaska. Talkeetna Formation about $200 \mathrm{ft}$ (61 m) above horizontal conglomerate. Earliest Sinemurian.

$26 \quad 48$ AI119 R. W. Imlay and D. J. Miller, 1948. Sea cliffs 2.95 miles $(4.7 \mathrm{~km}) \mathrm{S} .67^{\circ} \mathrm{W}$. of Point Naskowhak on south shore of Kachemak Bay, probably NE. cor. sec. 5 , T. 9 S., R. 15 W., Seldovia (B-5) quadrangle, on Kenai Peninsula, southern Alaska. Talkeetna Formation in gray limestone and green tuffaceous sandstone. Early Hettangian.
TABLE 7.-Description of Lower Jurassic fossil localities in Alaska-Continued

\begin{tabular}{|c|c|c|c|}
\hline $\begin{array}{l}\text { Locality No. } \\
\text { (figs. 2-6) }\end{array}$ & $\begin{array}{l}\text { USGS } \\
\text { Mesozoic } \\
\text { loc. No. }\end{array}$ & $\begin{array}{l}\text { Collector's } \\
\text { field No. }\end{array}$ & $\begin{array}{l}\text { Collector, year of collection, description of locality, } \\
\text { and stratigraphic assignment }\end{array}$ \\
\hline 26 & 664 & $1 \mathrm{AGz} 21$ & $\begin{array}{l}\text { Arthur Grantz, 1951. Sea cliffs } 2.8 \text { miles }(4.5 \mathrm{~km}) \\
\text { west of Point Naskowhak on south shore of } \\
\text { Kachemak Bay, near point on west side of sec. } \\
34 \text {, T. } 8 \text { S., R. } 16 \text { W., Seldovia (B-5) quadrangle, } \\
\text { Kenai Peninsula, southern Alaska. Talkeetna } \\
\text { Formation. Early Hettangian. }\end{array}$ \\
\hline
\end{tabular}

26 _ 31128 _ $35 \mathrm{JK}-77 \mathrm{~B}_{\text {_-- }}$ J. S. Kelley, 1976. Cliff near head of small bay southwest of Point Naskowhak in NE $1 / 4 \mathrm{NW}^{1 / 4}$ sec. 36, T. 15 S., R. 14 W., Seldovia (B-5) quadrangle, Kenai Peninsula, southern Alaska. Early Sinemurian.

$2676 \mathrm{JK}-15 \mathrm{P} \quad$ J. S. Kelley, 1976 . Sea Cliffs, 1.2 miles $(1.9 \mathrm{~km})$ $5^{\circ} \mathrm{SE}$. of Pt. Naskowhak, in NW1/4 SW $1 / 4$ sec. 31 , T. 8 S., R. 14 W., lat $59^{\circ} 26^{\prime} 45^{\prime \prime}$ N., long $151^{\circ} 44^{\prime \prime} 25^{\prime \prime}$ W., Seldovia (B-5) quadrangle, Kenai Peninsula, southern Alaska. Unnamed beds. Early Sinemurian.

26 ____ 31637 _ ___ 76 JK-16J___ J. S. Kelley, 1976 . Sea cliffs. 0.6 mile $(0.94 \mathrm{~km}) 6^{\circ}$ SE. of Pt. Naskowhak in NW1/4 NW $1 / 4$ sec. $31, \mathrm{~T}$. 8 S., R. 14 W., lat $59^{\circ} 26^{\prime} 71^{\prime \prime}$ N., long $151^{\circ} 44^{\prime \prime} 26^{\prime \prime}$ W., Seldovia (B-5) quadrangle, Kenai Peninsula, southern Alaska. Early Sinemurian.

$26 \ldots 31640 \ldots$ ______ 76 KK-21D __ J. S. Kelley, 1976 . Sea cliffs, 0.19 mile $(0.3 \mathrm{~km}) 14^{\circ}$ SE. of Pt. Naskowhak, NE $1 / 4 \mathrm{SE}^{1 / 4}$ sec. 25, T. 8 S., R. 14 W., lat $59^{\circ} 27^{\prime} 22^{\prime \prime}$ N., Long $151^{\circ} 44^{\prime} 50^{\prime \prime}$ W., Seldovia (B-5) quadrangle, Kenai Penin sula, southern Alaska. Unnamed beds. Early Sinemurian.

26 SW. of Pt. Naskowhak in NE $1 / 4 \mathrm{SW}^{1 / 4}$ sec. 34, T. 8 S., R. 15 W., lat $59^{\circ} 26^{\prime} 30^{\prime \prime}$ N., long $151^{\circ} 48^{\prime \prime} 86^{\prime \prime}$ W. Seldovia (B-5) quadrangle, Kenai Peninsula, southern Alaska. Unnamed beds. Early Hettangian.

26 ____ 31650 _____ H257_____. D. Mopkins, 1962. Sea cliffs just west of buried valley of Miocene age, lat $59^{\circ} 26.6^{\prime}$ N., long $151^{\circ} 47.1^{\prime}$ W. probably from same places as USGS Mesozoic loc. 2981, Seldovia (B-5) quadrangle, Kenai Peninsula, southern Alaska. Unnamed beds. Early Sinemurian.

$27 \quad 3109 \quad 949 \quad$ T. W. Stanton, 1904. Northeast shore of Puale Bay, about 1 mile $(1.6 \mathrm{~km})$ northwest of Chignik Point, Alaska Peninsula. A short distance southwest of locality 3110 and midway between that locality and Triassic Mesozoic loc. 19806 that contains Monotis. Unnamed beds. Hettangian.

$273110 \quad 950 \quad$ T. W. Stanton, 1904. Northeast shore of Puale Bay about 1 mile $(1.6 \mathrm{~km})$ northwest of Chignik Point and 5,400 ft $(1,646 \mathrm{~m}) \mathrm{N} .18^{\circ} \mathrm{W}$. of most easterly point of Puale Bay, Karluk (C4-C5) quadrangle, Alaska Peninsula. Unnamed beds. Hettangian.

27 _ $10820 \ldots$ S. R. Capps, 1921. Northeast shore of Puale Bay about $1 \frac{113}{3}$ miles $(1.8 \mathrm{~km})$ northwest of Chignik Point. Contains Paracaloceras as at Mesozoic loc. 12396 and ROC loc. 1241 but also contains one specimen of Schlotheimia preserved in a different matrix and some Late Triassic mollusks. Unnamed beds. Early Sinemurian (mostly). Some late Hettangian and Late Triassic.

27 W. R. Smith, 1923. Southwest shore Alinchak Bay, Karluk (D-4) quadrangle, Alaska Peninsula. Probably same place as locality ROC 3002 and USGS Mesozoic loc. 29268. Unnamed beds. Early and middle Hettangian.

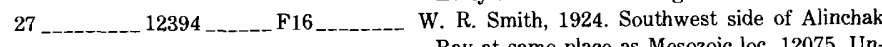
Bay at same place as Mesozoic loc. 12075. Unnamed beds, middle Hettangian.

27 ___ 12396 W. R. Smith, 1924. From northeast shore of Puale Bay reportedly about 2 miles $(3.2 \mathrm{~km})$ northwest of Chignik Point, Karluk (C4-C5) quadrangle, Alaska Peninsula. Apparently obtained from different units as shown by presence 
TABLE 7.-Description of Lower Jurassic fossil localities in Alaska-Continued

\begin{tabular}{cccc}
$\begin{array}{c}\text { Locality No. } \\
\text { (figs. 2-6) }\end{array}$ & $\begin{array}{c}\text { USGS } \\
\text { Mesozoic } \\
\text { loc. No. }\end{array}$ & $\begin{array}{c}\text { Collector's } \\
\text { field No. }\end{array}$ & $\begin{array}{c}\text { Collector, year of collection, description of locality, } \\
\text { and stratigraphic assignment }\end{array}$ \\
\hline
\end{tabular}

of Paracaloceras as at USGS Mesozoic loc 10820 and of Waehneroceras as at Mesozoic locality 3110 . Unnamed beds. Early Sinemurian and late Hettangian.

27 (44 AKm F75) L. B. Kellum, S. N. Daviess, and C. M. Swinney, 1944. Northeast shore of Puale Bay 1 mile $(1.6 \mathrm{~km})$ northwest of Chignik Point, Alaska Peninsula. From dense limestone in terbedded with limy shale, and some tuffaceous sandstone $360 \mathrm{ft}(110 \mathrm{~m})$ below base of massive tuff and agglomerate. Unnamed beds. Middle Hettangian.

$27 \ldots 19804 \ldots 4$ AKm F 76 L. B. Kellum, S. N. Daviess, and C. M. Swinney, 1944. On northeast shore of Puale Bay about 2 miles $(3.2 \mathrm{~km})$ north-northeast of Chignik Point and $1,000 \mathrm{ft}(305 \mathrm{~m})$ north of northernmost large waterfall, Karluk (C-4 and C-5) quadrangles, Alaska Peninsula. In dark-gray shale $30 \mathrm{ft}(9 \mathrm{~m})$ above base of Kialagvik Formation. Near middle of Toarcian.

27 _____ 21237 ____ 48AI 112___ R. W. Imlay and D. J. Miller, 1948. Northeast shore of Puale Bay, Alaska Peninsula, 1.63 miles $(2.6 \mathrm{~km}) \mathrm{N} .22^{\circ} \mathrm{W}$. of Chignik Point. Float probably from light-gray sandstone exposed in cliffs about $340 \mathrm{ft}(103 \mathrm{~m})$ below base of Kialagvik Formation. Unnamed beds. Early to early late Sinemurian.

$27 \ldots$ _.___ 25694 _____ 55AKe6 _____ A. S. Keller, 1955. Southwest side of Alinchak Bay in limestone overlying Triassic beds. Probably same place as 12075,12394 , and 25694. Unnamed beds. Middle Hettangian.

27 _.____ 29267 ____ 65 175___ Marvin Mangus, 1965. Northeast shore of Puale Bay about 1 mile $(1.6 \mathrm{~km})$ northwest of Chignik Point, Alaska Peninsula. Probably from same place as Mesozoic locs. 3110 and 19803. Unnamed beds. Middle Hettangian.

27 ___ 29268 ____ 65 AMe55 ___ G. Woore, 1965. Southwest shore of Alinchak Bay, lat $57^{\circ} 45.8^{\prime} \mathrm{N}$., long $155^{\circ} 18.9^{\prime} \mathrm{W}$., Karluk (D-4) quadrangle, Alaska Peninsula. Unnamed beds. Middle Hettangian.

27 ________ M1738______ALp701M___ British Petroleum, Inc., 1962. From or near the same place as Mesozoic locs. 3110 and 19803 , $3^{1 / 2}$ miles $(5.6 \mathrm{~km})$ west of Cape Kekurnoi, lat $57^{\circ} 43.4^{\prime}$ N., long $155^{\circ} 23.5^{\prime}$ W., east shore or Puale Bay, Alaska Peninsula. Unnamed beds. Early to middle Hettangian.

$27+31370 \quad$ 77AI1___ R. W. Imlay, Martha Yount, Carleen Holloway, and Fred Wilson, 1977. Northeast side of Puale Bay. Same place as USGS Mesozoic loc. 3109 , about $200 \mathrm{ft}(61 \mathrm{~m})$ stratigraphically above top of Monotis-bearing beds, 1 miles $(1.6 \mathrm{~km})$ northwest of Chignik Point, Karluk (C4-C5) quadrangle, Alaska Peninsula. Unnamed beds. Hettangian.

$27 \quad 31372 \ldots 77 \mathrm{AI} 3 \ldots \ldots$ R. W. Imlay, Martha Yount, Carleen Holloway, and Fred Wilson, 1977. Northeast side of Puale Bay. About $4,300 \mathrm{ft}(1,311 \mathrm{~m}) \mathrm{S} .12^{\circ} \mathrm{E}$. of VABM Bay 119. About 270 to $280 \mathrm{ft}(82-86 \mathrm{~m})$ stratigraphically above base of massive tuffaceous sandstone. Karluk (C4-C5) quadrangle, Alaska Peninsula. Early Sinemurian.

27 ROC 1185__ W. T. Rothwell and associates, 1962. From 1,900 ft $(1,494 \mathrm{~m}) \mathrm{N} .30^{\circ} \mathrm{W}$. of VABM 96 Hike on northeast shore of Puale Bay, Alaska Peninsula. Float from or near same place as localities 3110 and 19803 . Unnamed beds. Early to late Hettangian.

27 ROC $1240 \ldots$ W. T. Rothwell and associates, 1962. From 10 ft $(3 \mathrm{~m})$ stratigraphically below locality $\mathrm{ROC}$ 1241 and probably 270 to $280 \mathrm{ft}(82-86 \mathrm{~m})$ stratigraphically above base of tuffaceous sandstone. Unnamed beds. Early Sinemurian.

27 ROC 1241__. W. T. Rothwell and associates, 1962. Northeas shore of Puale Bay. About $4,300 \mathrm{ft}(1,311 \mathrm{~m}) \mathrm{S}$.
TABLE 7.-Description of Lower Jurassic fossil localities in Alaska-Continued

\begin{tabular}{cccc}
\hline $\begin{array}{c}\text { Locality No. } \\
\text { (figs. 2-6) }\end{array}$ & $\begin{array}{c}\text { USGS } \\
\text { Mesozoic } \\
\text { loc. No. }\end{array}$ & $\begin{array}{c}\text { Collector's } \\
\text { field No. }\end{array}$ & $\begin{array}{c}\text { Collector, year of collection, description of locality, } \\
\text { and stratigraphic assignment }\end{array}$ \\
\hline
\end{tabular}

$12^{\circ}$ E. of VABM 119 Bay, Karluk (C4-C5) quadrangle, Alaska Peninsula. About $700 \mathrm{ft}$ (213 m) below base of Kialagvik Formation. Unnamed beds. Early Sinemurian.

27

W. T. Rothwell and associates, 1962. Same general location as Mesozoic loc. 19804. At point about $3,000 \mathrm{ft}(914 \mathrm{~m}) \mathrm{S} .12^{\circ} \mathrm{E}$. of VABM 119 Bay $(\mathrm{N}=$ bed north of fault, $\mathrm{S}=$ bed south of fault). In massive sandstone about $290 \mathrm{ft}(88 \mathrm{~m})$ below the base of the Kialagvik Formation. Unnamed beds. Late early to eariy late Sinemurian.

27 ROC $1283_{-\ldots}$ W. T. Rothwell and associates, 1962. Same description as locality ROC 1282 but $10 \mathrm{ft}(3 \mathrm{~m})$ lower in the massive sandstone. Unnamed beds. Late early to early late Sinemurian.

27 ROC 1303_-_ W. T. Rothwell and associates, 1962. Same general location as Mesozoic loc. 19804. At point about $2,700 \mathrm{ft}(823 \mathrm{~m}) \mathrm{S} .12^{\circ} \mathrm{E}$. of VABM 119 Bay. From unit of massive sandstone $160 \mathrm{ft}$. (49 m) thick underlying the Kialagvik Formation. Unnamed beds. Sinemurian.

27

W. T. Rothwell and associates, 1962 . South end of Alinchak Bay about $2.1 \mathrm{mi}(3.3 \mathrm{~km}), \mathrm{N} .35^{\circ} \mathrm{E}$. of VABM 1197 Kek, Karluk (D-4) quadrangle, Alaska Peninsula. Unnamed beds. Early and middle Hettangian.

28 Richfield Oil Co., Wide Bay Test Well 1, core 5 at depth of 2,235 to $2,236 \mathrm{ft}(681 \mathrm{~m})$, at Wide Bay, Alaska Peninsula. Unnamed beds. Hettangian.

\section{SYSTEMATIC DESCRIPTIONS}

\section{Family PSILOCERATIDAE Hyatt, 1867 Genus PSILOCERAS Hyatt, 1867}

Psiloceras cf. P. planorbis (J. de C. Sowerby)

Plate 1, figures 1,2

Ten compressed molds represent a highly evolute smooth ammonite similar to $P$. planorbis (J. de C. Sowerby) (1824, pl. 448; Dean and others, 1961, pl. 63, fig. 1; Arkell and others, 1957, p. L232, fig. 258-10, a-c) of early Hettangian Age. Their occurrence with Waehneroceras of middle Hettangian Age in three collections implies either that some of the specimens herein compared with $P$. planorbis are of that age or that the collections were made from more than one stratigraphic unit. The presence of beds of earliest Hettangian Age in the areas where these collections were made is shown by the upward gradation of marine Upper Triassic beds into marine Lower Jurassic beds.

Figured specimens.-USNM (U.S. National Museum) $247950,247951$.

Occurrences. - Upper member of McCarthy Formation at USGS Mesozoic loc. 29891 in the Wrangell Mountains; unnamed beds on the small peninsula between Puale Bay and Alinchak Bay, Alaska Peninsula, at USGS Mesozoic locs. 12075 and M1738 and ROC (Richfield Oil Co.) localities 1185 and 3002. 


\author{
Subgenus FRANZICERAS Buckman, 1923 \\ Psiloceras (Franziceras) cf. P. (F.) ruidum (Buckman)
}

Plate 1, figures 12-14, 18-24

Twelve external molds of immature ammonites are fairly evolute, have a subovate whorl section that is a little higher than wide, an umbilicus that represents about 50 percent of the diameter, and rather sharp, widely spaced ribs. These ribs begin near the umbilicus, trend radially or slightly adapically on the flanks, and terminate rather abruptly a little above the middle of the flanks. In addition, faint lines that trend radially on the flanks and arch forward on the venter are preserved on shelly material that occurs on a few specimens from the Seldovia area.

The suture line is simple, has fairly narrow saddles, and does not have a strongly retracted suspensive lobe.

This species differs from plicate forms of Psiloceras such as $P$. (P.) plicatum (Quenstedt) (1883, pl. 1, figs. 9, 11; Donovan, 1952, pl. 22, figs. 1, 2; Lange, 1941, pl. 2, fig. 20) by having sharp ribs instead of folds. It differs from species of the subgenus $P$. (Caloceras) by being less evolute, by having a smaller umbilicus relative to its diameter, by ribs trending radially instead of projecting adorally, and by having a somewhat different sutural pattern. It appears to have somewhat weaker ribbing than the fragmentary specimens of Psiloceras from the northern Yukon that were described by Frebold and Poulton (1977, p. 92, 93, pl. 1 figs. 3-8). Overall it shows most resemblance to $P$. (Franziceras) ruidum Buckman (1923, pl. 423) but differs by having shorter ribs and a less retracted suspensive lobe. These differences may be related to its much smaller size.

It is associated at USGS Mesozoic loc. 29737 with small specimens of Psiloceras, of which some are smooth and some bear faint ribs on their innermost whorls.

Figured specimens.-USNM 247952-247955.

Occurrences.-Glenn Shale in Coleen quadrangle of east-central Alaska at USGS Mesozoic locs. 29737-29739 and 29742; unnamed beds in the Seldovia area on the Kenai Peninsula at USGS Mesozoic locs. 21242,22664 , and 31648 .

\section{Psiloceras (Franziceras) sp. ind.}

Plate 1, figures 11, 15-17

This species is represented by two specimens. It differs from the species described herein as $P$. $(F$.) cf. $P$. $(F$.) ruidum (Buckman) by having sparser, shorter ribs that are much more prominent on the lower third of the flanks. These ribs are particularly prominent near the umbilical margin on the inner whorls of a specimen from northernmost Alaska as well as on the smaller speciment from east-central Alaska. On the outer whorl of the specimen from northernmost Alaska, the ribs fade considerably near the middle of the flanks but persist across the venter, where they arch forward and vary from weak to fairly strong. In addition, fine closely spaced radial striae are present on some shelly material that is preserved on the inner whorls of the larger specimen. The suture line is not preserved.

Figured specimens.-USNM 247956, 247957.

Occurrences.-Glenn Shale in the Coleen quadrangle of east-central Alaska at USGS Mesozoic loc. 29742; Kingak Shale in northernmost Alaska in the South Barrow No. 12 well at the depth of 2,170.5 feet $(661.4 \mathrm{~m})$.

\section{Genus DISCAMPHICERAS Spath, 1929 \\ Discamphiceras cf. D. toxophorum (Waehner) \\ Plate 1, figures 3, 4, 8-10}

This species is represented by 12 specimens, of which 10 are crushed laterally. It is characterized by moderately involute coiling; an umbilicus that is about two-fifths as wide as the shell diameter; a high whorl section; a narrow, fairly sharp venter, and by moderately spaced ribs that trend radially, or incline slightly forward on the flanks, and that arch forward on the ventral area of smaller specimens. These ribs broaden and weaken ventrally and are faint or absent on venters of medium to large specimens. On the largest specimen the adapical half bears widely spaced ribs that fade out ventrally, whereas the adoral half is completely smooth. The suture line is not preserved.

This species closely resembles $D$. toxophorum Waehner (1884, p. 109, pl. 24, figs. 5a,b to 7a-c) in most respects but has somewhat sparser ribbing. The small specimens differ from comparable specimens of $D$. kammerkahrense (Gümbel) (Waehner, 1884, p. 113, pl. 24, figs. 3a-c, 4a-d) and from $D$. calcimontanum (Waehner) (1884, p. 112, pl. 24, figs. 1a-c, 2a,b) by having a wider umbilicus and sharper, sparser ribbing. The ribbing on the outer whorl of the largest Alaskan specimens is somewhat similar to that on the largest specimens of $D$. kammerkahrense (Gümbel) (Waehner, 1884, pl. 25, figs. $1 \mathrm{a}-\mathrm{c})$ and of $D$. calcimontanum (Waehner) $(1884, \mathrm{pl} .24$, figs. 1a,b).

Assignment of these Alaskan specimens to Discamphiceras rather than Waehneroceras is favored by their moderately involute coiling, by their ribs fading on the venter of small specimens, and by their adult outer whorl being fairly smooth.

Figured specimens.-USNM 247958-247961.

Occurrences.-Unnamed beds on peninsula between Puale Bay and Alinchak Bay on Alaska Peninsula at USGS Mesozoic locs. 3110, 25694, M1738, and ROC loc. 3002.

\section{Discamphiceras sp. \\ Plate 1, figures 5-7}

Seven laterally crushed molds differ from the Alaskan specimens herein compared with Discamphiceras tox- 
ophorum (Waehner) by having weaker and more closely spaced ribs. In that respect they resemble a small specimen of $D$. calcimontanum (Waehner) $(1884$, p. 112 , pl. 24, figs. 2a, b.) Their ribs are much weaker and do not extend as far ventrally as on the type specimens of $D$. toxophorum (Waehner) (1884, p. 109, pl. 24, figs. 5a-c to $7 \mathrm{a}-\mathrm{c})$.

Figured specimens.-USNM 247962.

Occurrence.-Upper member of the McCarthy Formation in the Wrangell Mountains at USGS Mesozoic loc. 29890.

\section{Genus LAQUEOCERAS Lange, 1925 \\ Laqueoceras cf. L. sublaqueus (Waehner) \\ Plate 3, figure 13}

This species is represented by one laterally crushed specimen that includes a large internal mold and a small, shell-bearing fragment. The specimen is highly evolute, consists of many whorls, and bears fine radial, rather closely spaced ribs. These ribs are fairly distinct on the inner whorls at diameters as large as $75 \mathrm{~mm}$ but gradually become faint at greater diameters and are barely evident on the outermost whorl. The venter is poorly exposed but apparently does not bear a keel.

The specimen is similar to L. sublaqueus (Waehner) (1886, p. 142, pl. 15, fig. 2; pl. 16, fig. 10; pl. 30, fig. 4; Donovan, 1952, p. 641,642 ) in size and in the strength, closeness, and persistence of its ribbing.

Figured specimen.-USNM 247963

Occurrence.-Unnamed Lower Jurassic beds on south side of Alinchak Bay on Alaska Peninsula at ROC loc. 3002 .

\section{Genus WAEHNEROCERAS Hyatt, 1889}

Waehneroceras cf. W. tenerum (Neumayr)

Plate 2, figures 1-6

cf. Aegoceras tenerum Neumayr, 1879, K. K. Geol. Reichsanst., Abh. v. 7, p. 31 , pl. 3 , figs. 4,5 .

cf. Waehneroceras tenerum (Neumayr). Arkell and others, 1957,

Treatise on invertebrate paleontology, Part L, Mollusca 4, p. L235, fig. 260-1a,b.

This species is represented by 30 crushed molds. It has highly evolute coiling, a wide umbilicus, and an ovate whorl section. Its ribs are widely spaced, trend nearly radially on the flanks, become stronger ventrally, project forward and become much weaker on the margin of the venter, and are reduced in strength along the midline of the venter.

This species differs from the specimens herein compared with $W$. portlocki (Wright) by having a lower whorl section and much stronger and sparser ribbing. Its features are essentially the same as those of $W$. tenerum (Neumayr) (Arkell and others, 1957, p. L235, fig. 260-1a,b). It is associated at four localities in Alaska with Waehneroceras cf. W. portlocki (Wright) and at three localities with Discamphiceras.
Figured specimens.-USNM 247964-247966.

Occurrences.-Unnamed beds on south side of Alinchak Bay on Alaska Peninsula at USGS Mesozoic locs. 12075, 12394, 25694, 29268 and ROC loc. 3002; unnamed beds on east side of Puale Bay on Alaska Peninsula at USGS Mesozoic locs. 3109, 3110, 19803, 31370, and ROC loc. 1185.

\section{Waehneroceras cf. W. portlocki (Wright)}

Plate 2, figures 7, 10-15

cf. Aegoceras portlocki Wright, 1881, 1882, London, Palaeontographical Soc., l. pl. 48, figs. 4, 5 (1881), p. 372 (1882).

cf. Aegoceras extracostatum Waehner, 1882, Beitr. Pal. Geol. OsterUngarns v. Orient, v. 2, pt. 3, p. 74, pl. 14, fig. 1.

cf. Macrogrammites grammicus Buckman, 1928, Type ammonites, v. 7 , pl. 761 a,b.

cf. Schlotheimia (Waehneroceras) portlocki (Wright). Donovan, 1952, Annals and Mag. Nat. History, ser. 12, v. 5, p. 646, pl. 22, figs. 5a,b. cf. Schlotheimia (Waehneroceras) portlocki (Wright). Dean, Donovan, and Howarth, 1961, British Mus. (Nat. History) Bull., v. 4, no. 10, p. 445 , pl. 63 , figs. $4 \mathrm{a}, \mathrm{b}$.

This species is represented by 33 internal and external molds, of which most are laterally crushed. These specimens have highly evolute coiling, a wide umbilicus, and a compressed whorl section. Their ribs trend nearly radially on the flanks, bend forward slightly on the ventral margin, are rather closely spaced on septate whorls and become more widely spaced on the incomplete body chamber. The venter is smooth along its midline as shown on several specimens. The suture line is not exposed.

In coiling and ribbing this species is essentially identical with the specimen of Waehneroceras portlocki (Wright) figured by Dean, Donovan, and Howarth (1961, pl. 63, figs. 4a,b) and probably represents the same species. None of the Alaska specimens, however, include the large outer whorl as figured by Wright $(1881, \mathrm{pl} .48$, figs. 4, 5) and Buckman (1928, pl. 761a,b). W. curviornatum (Waehner) (1882, p. 75, pl. 16, figs. 2-4) appears to have slightly coarser ribbing on its inner whorls and develops a smooth venter on its outer whorls.

Figured specimens.-USNM 247967-247972.

Occurrences.-Unnamed beds on south side of Alinchak Bay on Alaska Peninsula at USGS Mesozoic locs. 12075,12394 , 25694, and 29268, and ROC loc. 3002; unnamed beds on east side of Puale Bay on Alaska Peninsula at Mesozoic loc. 3110, 29267 and 31370; unnamed beds in Richfield Oil Co.-Wide Bay test well No. 1, core no. 5 at depth of 2235 to $36 \mathrm{ft}(681 \mathrm{~m})$ on north side of Wide Bay, Alaska Peninsula.

Waehneroceras? sp.

Plate 2, figures 8,9

Waehneroceras is possibly represented in the subsurface of northern Alaska by one small fragment of a whorl in which only the venter and upper part of the 
flank are preserved. The specimen is characterized by simple ribs that project strongly forward on the venter and that are only slightly reduced in strength on the venter. These features are similar to those on immature specimens of Waehneroceras tenerum (Neumayr) (Arkell and others, 1957, p. L235, figs. 1a,b).

Figured specimen.-USNM 247973.

Occurrence.-Kingak Shale in South Barrow test well No. 12 at depth of $2181.5 \mathrm{ft}$. (665 m) in northern Alaska. This occurrence is $11 \mathrm{ft}(3.3 \mathrm{~m})$ below that of Psiloceras (Franziceras).

\section{Family SCHLOTHEIMIIDAE Spath, 1923 Genus SCHLOTHEIMIA Bayle, 1878 Schlotheimia sp. \\ Plate 2, figures 16,17}

One specimen is moderately evolute. Its whorl section is a little higher than it is wide. The umbilical width is 40 percent of the diameter. Its ribs are simple, sharp, incline forward on the flanks, become a little stronger ventrally, arch forward on the venter, and are only slightly reduced in strength along the midline of the venter.

The ribbing on this specimen resembles that on the inner whorls of $S$. montana (Waehner) (1886, p. 165, pl. 19, fig. 1; pl. 20, fig. 1) and of $S$. donar (Waehner) (1886, p. 173, pl. 21, figs. 2, 5).

Figured specimens.-USNM 247974.

Occurrence.-Unnamed beds in the Puale Bay area on Alaska Peninsula at USGS Mesozoic loc. 10820.

Genus BADOUXIA Guex and Taylor, 1976

Badouxia canadensis (Frebold)

Plate 2, figures 18-21, 24-28

Psiloceras canadense Frebold, 1951, Canada Geol. Survey Bull. 18, p. 3, pl. 1, figs. 1-6, pl. 2, fig. 1a-c; pl. 3, fig. 1 .

Psiloceras canadense Frebold, 1964a, Canada Geol. Survey Bull. 116, p. 6, pl. 1, figs. 1-5b.

Psiloceras canadense Frebold, 1967b, Canada Geol. Survey Bull. 158, p. 18, pl. 1, figs. lab, 2a,b, 3a-c.

Badouxia canadensis (Frebold). Guex and Taylor, 1976, Eclogae

Geol. Helvetiae, v. 69, no. 2, p. 525.

This species is represented in Alaska by 10 fairly small specimens. Most of these (pl. 2, figs. 18, 24, 28) bear rather closely spaced ribbing comparable with that on certain specimens illustrated by Frebold (1951, pl. 1, fig. 5a; 1967b, pl. 1, fig. 2a). A few specimens (pl. 2, figs. 20, 26,27 ) bear sparser ribbing, as does another specimen illustrated by Frebold (1951, pl. 1, figs. 1e,d; 1967b, pl. 1 fig. 3a).

The species has ovate whorls that are higher than they are wide, embrace half or more of the preceding whorl, and are rounded ventrally. Its smaller whorls bear straight, fairly sharp, forwardly inclined ribs that extend across the lower two-thirds of the flanks and then either fade out rather abruptly or pass into very weak secondary ribs. Its larger whorls are marked only by strong forwardly inclined ribs that fade out rather abruptly at the base of the upper third of the flanks. Its assignment to the genus Psiloceras is questioned because it is more involute and more strongly ribbed than is typical of that genus. Its ribbing is much stronger on the lower part of the flanks than in Arctoasteroceras jeletzkyi Frebold.

Hypotypes. - USNM 247975-247977 and 248062.

Occurrences.-Unnamed beds in the Healy (A-6) quadrangle in Alaska Range at USGS Mesozoic loc. 31266; unnamed beds at Puale Bay in Alaska Peninsula at ROC loc. 1240, and USGS Mesozoic loc. 31372.

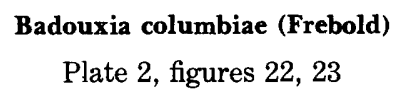

Psiloceras (Curviceras) columbiae Frebold, 1967b, Canada Geol.

Survey Bull. 158, p. 20, pl. 1, figs. 10a-c; pl. 2, figs. 1-5;

p. 3, figs. 2a-c.

Schlotheimia? sp. indet. Frebold, 1951, p. 7, pl. 4, figs. 2-4.

One fragmentary ammonite bears simple ribs that incline forward on the flanks as do those on $B$. canadensis (Frebold), but that continue across the venter where they arch strongly forward as in B. columbiae (Frebold).

Figured specimen.-USNM 247978.

Occurrence.-Unnamed beds in the Healy (A-6) quadrangle, Alaska Range at USGS Mesozoic loc. 31264.

\section{Genus CHARMASSEICERAS Spath, 1924 \\ Charmasseiceras cf. C marmoreum (Oppel)}

Plate 3, figures 1-3

This species is represented by three fragmentary molds of immature specimens. The best preserved internal mold shows that the species is fairly involute, that its ribbing changes during growth from fairly fine to moderately strong, that many ribs fork low on the flanks, and that all ribs curve forward on the upper parts of the flanks. One external mold bears similar forwardly curved ribs that terminate ventrally in shelly material whose smoothness suggest a ventral band.

These specimens are closely similar in involution and ribbing to the small specimen of Charmasseiceras marmoreum (Oppel) figured by Waehner (1886, pl. 22, figs. $2-4)$ and by Frebold (1967b, pl. 3, figs. 1c and 1d; pl. 4, figs. 2a-c).

Figured specimens.-USNM 247979.

Occurrence.-Kingak Shale in northern Alaska in the South Barrow test well No. 12 at depths of 2061.5 and 2068 feet (628 and $630 \mathrm{~m})$. Unnamed beds in Seldovia area in northern Alaska at USGS Mesozoic locs. 2979 and 31637.

\section{Charmasseiceras sp. \\ Plate 3, figures 4-10}

Three small specimens are characterized by a subquadrate whorl section that is much higher than it is 
wide; by a flattened venter; by high, sharp, widely spaced ribs that incline forward strongly on the upper parts of the flanks; and by prominent forwardly inclined ventral swellings that on the largest specimen are joined across the venter by low weak chevron-shaped ribs.

These specimens differ from immature specimens of C. marmoreum (Oppel) (Waehner, 1886, pl. 22 figs. 2-6) by having slightly coarser ribs of which only a few fork low on the flanks. In whorl shape and ribbing, the specimens show more resemblance to some small specimens that were figured as $C$. charmassei (d'Orbigny) (1844, pl. 91, figs. 1,2). They also resemble some small specimens of $C$. posttaurinum (Waehner) (1886, pl. 23 , figs. $17 \mathrm{a}-\mathrm{c}$ ) in ribbing but are much more compressed.

Figured specimens.-USNM 247980, 247981.

Occurrences.-Kingak Shale in northern Alaska in the South Barrow test well No. 12 at depth of 2,056 feet $(627 \mathrm{~m})$ and in the South Barrow test well No. 3 at depths of 2,412 and 2,419.5 feet (735 and $737 \mathrm{~m}$ ).

Family ARIETITIDAE Hyatt, 1874

Subfamily ARIETITINAE Hyatt, 1874

Genus ARIETITES Waagen, 1869

Arietites cf. A. bucklandi (J. Sowerby)

Plate 4, figures 11-13

"Arietites" cf. "A." bucklandi (J. Sowerby). Imlay, 1955, U.S. Geol. Survey Prof. Paper 274-D, p. 87, pl. 10, figs. 7, 8.

The assignment of this species to Arietites was accepted by Arkell (1956, p. 530). No other specimen of the genus has yet been found in Alaska.

Figured specimen.-USNM 108778.

Occurrence.-Kingak Shale in northern Alaska in the Avak test well No. 1 at depth of $1836 \mathrm{ft}(560 \mathrm{~m})$.

\section{Genus CORONICERAS Hyatt, 1867}

Coroniceras sp. A

Plate 4, figures 1-5

This species, represented only by fragmentary material, is similar in appearance to Coroniceras multicostatum (J. de C. Sowerby) (1824, v. 5, p. 75, pl. 454; Reynes, 1879, pl. 25, figs. 1-2; Guerin-Franiatte, 1966, p. 141, pls. 29-32). The specimens from Alaska are fairly evolute. Their whorls are higher than they are wide. Their ribs are fairly strong, radial on the flanks, bend forward on the ventral margin, and bear ventrolateral tubercles. Their keel is fairly strong and on internal molds is bordered by shallow furrows.

Figured specimen.-USNM 247982.

Occurrence.-Unnamed Lower Jurassic beds in Talkeetna (C-6) quadrangle, central part of the Alaska Range at USGS Mesozoic loc. 30908.

\author{
Coroniceras sp. B \\ Plate 4 , figures $6-10$
}

This species is represented by 12 poorly preserved internal and external molds. Its appearance is similar to that of the specimens herein described as Coroniceras sp. A, but differs by having ribs curving forward much more strongly on the upper part of its flanks. In that respect it shows more resemblance to $C$. haueri (Waehner) (1886, p. 38, (127), pl. 19, fig. 1a,b; pl. 20, fig. $2 a, b)$. Its ribbing also resembles some species of Caenesites (J. de C. Sowerby, 1824, pl. 452, fig. 1; Wright, 1879, pl. 12, fig. 1; Arkell, 1956, pl. 31, fig. 1, Dean and others, 1961, pl. 66, figs. 1,2; GuerinFraniatte, 1966, pls. 204, 208). It differs from that genus, however, in that its keel is bordered by very weak instead of deep furrows.

Figured specimens.-USNM 247983, 247984.

Occurrences.-Kingak Shale in northern Alaska in the South Barrow test well No. 12 at depth of 1987.4 to $1987.6 \mathrm{ft}(606 \mathrm{~m})$.

\section{Coroniceras sp. C \\ Plate 4, figure 14}

Three small fragmentary molds of ammonites from the subsurface of northern Alaska possibly represent the inner whorls of the species described herein as Coroniceras sp. A. They appear, however, to have slightly sparser ribs that bear stronger tubercles on the ventrolateral margin. The keel is fairly low and is not bordered by furrows.

Figured specimens.-USNM 247985.

Occurrence.-Kingak Shale in northern Alaska in the South Barrow test well No. 3 at depth of $2470 \mathrm{ft}(753 \mathrm{~m})$.

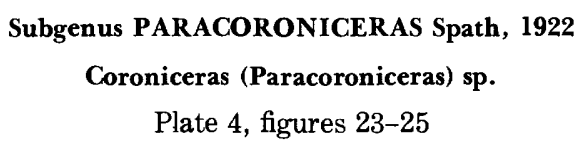

One species, represented by a crushed fragment of an outer whorl and by one internal mold, bears prominent widely spaced ribs that trend radially on the flanks and curve forward on the ventral margin. Its keel is fairly high and is not bordered by furrows. Its whorl section is considerably higher than wide.

This specimen resembles the adoral end of the outermost preserved whorl of the coarsely ribbed holotype of Coroniceras (Paracoroniceras) charlesi Donovan (1955, p. 12, 28; Reynes, 1879, pl. 16, figs. 1, 2; GuerinFraniatte, 1966, p. 153, pl. 38).

Figured specimen.-USNM 247986.

Occurrence.-Unnamed beds in the Seldovia area on Kenai Peninsula at USGS Mesozoic locs. 31128 and 31650 . 
Genus ARNIOCERAS Hyatt, 1867

Arnioceras cf. A. densicosta (Quenstedt)

Plate 5, figures 9-11, 16-24

This species is represented by 38 specimens, of which most are molds; but shelly material is preserved on some small specimens obtained from a well core in northern Alaska. It has highly evolute coiling. Its ribs are sharp, fairly closely spaced, trend radially on the flanks, bend forward on the margins of the venter, and then fade out on the venter. Its innermost whorls are smooth to a diameter of 15 to $20 \mathrm{~mm}$. Its venter bears a keel that is bordered by distinct furrows on the internal mold and by weak furrows where shelly material is preserved. These features are essentially identical with those on the lectotype of Arnioceras densicosta (Quenstedt) $(1858, \mathrm{pl} .7$, fig. 7; 1884, p. 100, pl. 13, fig. 7; Guerin-Franiatte, 1966, pl. 142, figs. 1a,b) and on other comparable illustrated specimens (Guerin-Franiatte, 1966, p. 265, pl. 142, figs. 2, 3; Reynes, 1879, pl. 14, figs. 5, 6).

The suture line is similar to that on $A$. densicosta Quenstedt (1884, pl. 13, fig. 7). The first lateral lobe is broad, bifid, and a little deeper than the ventral lobe. The first lateral saddle is bifid.

Figured specimens.-USNM 247987-247989.

Occurrences.-Unnamed beds in the Puale Bay area on Alaska Peninsula at USGS Mesozoic loc. 21237, and ROC locs. 1282N, ROC 1282S, and ROC 1283A; unnamed beds in the Healy (A-6) quadrangle, Chulitna Valley area in the Alaska Range at USGS Mesozoic locs. 16229, $31260,31262,31263,31265$. Upper member of McCarthy Formation in the Wrangell Mountains at USGS Mesozoic locs. 28535, 28538, and 30140.

\section{Arnioceras sp. juv. \\ Plate 5, figures 5, 6, 12-15}

Arnioceras is represented in northern Alaska by many small immature specimens obtained from a well core in the Point Barrow area. These specimens are nearly identical in coiling, whorl shape, and ornamentation with the inner whorls of the specimens herein described as $A$. cf. A. densicosta (Quenstedt). They differ by their innermost whorls, being smooth only to diameters of 8 to $15 \mathrm{~mm}$.

Figured specimens.-USNM 247990.

Occurrence.-Kingak Shale in northern Alaska in the South Barrow test well No. 12 at depth of $2056 \mathrm{ft}$ $(626.7 \mathrm{~m})$

\section{Genus PARACALOCERAS Spath, 1923 \\ Paracaloceras rursicostatum Frebold \\ Plate 6, figures 1-11}

Paracaloceras rursicostatum Frebold, 1967b, Canada Geol. Survey Bull. 158, p. 26, pl. 7, figs. 1a-c, 2a-c; pl. 9, fig. 1.

Ten laterally crushed internal molds are characterized by highly evolute coiling and by strong, rather widely spaced ribs that curve adapically on most flanks, curve adorally and become stronger on the ventral margin, and then terminate abruptly. The keel on the mold is fairly low and is bounded laterally by two furrows that are bounded by ridges. The furrows and the lateral ridges are very weak on the small specimens but are strong on the larger specimens (pl. 6, figs. 1, 5, 11). On the least crushed specimen (pl. 6, figs. 7, 11), the whorl section is nearly as wide as it is high. During growth, the adapical arching of the ribs becomes more pronounced.

All the specimens from Alaska have sparser and somewhat coarser ribbing than those described as Paracaloceras cf. P. coregonense (Sowerby) by Frebold (1951, p. 7, pl. 5, figs. 1-6; pl. 6, fig. 1; 1967b, p. 24, pl. 7, figs. 3-7). Their ribbing is similar, however, to some specimens illustrated by Waehner $(1888$, pl. 21 , figs. 1-2; pl. 23, fig. 4) as Arietites coregonensis Sowerby. They differ mainly by having ribs bending adapically more strongly on their largest whorls, a feature characteristic of $P$. rursicostatum Frebold.

Hypotypes.-USNM 247991-247997 and 248070.

Occurrences. - Unnamed beds in the Puale Bay area on Alaska Peninsula at USGS Mesozoic locs. 10820, 12396, 31372, and ROC loc. 1241; unnamed beds in the Seldovia area on Kenai Peninsula at Mesozoic loc. 2981; unnamed beds in the Healy (A-6) quadrangle in Alaska Range at Mesozoic loc. 31266.

\section{Genus ARCTOASTEROCERAS Frebold, 1960}

Arctoasteroceras jeletzkyi Frebold

Plate 5, figures 1-4

Arctoasteroceras jeletzkyi Frebold, 1960, Canada Geol. Survey Bull. 59 , p. 14, pl. 2, figs. 1-5; pl. 3, figs. 1-3.

Arctoasteroceras jeletzkyi Frebold, 1964b, Canada Geol. Survey Bull. $63-4$, p. 5, pl. 2, figs. 1, 2.

This species is represented in Alaska by two specimens, of which the larger closely resembles the holotype (Frebold, 1960, pl. 2, fig. 1a,b) and the smaller resembles a paratype (Frebold, 1960, pl. 3, fig. 3a,b). Resemblances include a moderately involute shell, an ovate whorl section, gently convex flanks, a low blunt keel, and moderately spaced ribs that incline forward on the lower two-thirds of the flanks and then bend forward and become faint on the upper third and on the venter. The only difference is the presence of faint sulci bordering the keel. The suture line is poorly preserved on the Alaska specimens.

Hypotypes.-USNM 247998, 247999.

Occurrences.-Unnamed beds in the Healy (A-6) quadrangle in Alaska Range at USGS Mesozoic loc. 31261 . 
Family ECHIOCERATIDAE Buckman, 1913 Genus PALTECHIOCERAS Buckman, 1924

Paltechioceras cf. P. harbledownense (Crickmay) Plate 4, figures, 15-22

cf. Melanhippites harbledownensis Crickmay, 1928, California Univ. Pub. Dept. Geol. Sci. Bull., v. 18, no. 2, p. 61, pl. 3, pl. 4a-d.

Ten specimens represent a species characterized by highly evolute coiling, an elliptical whorl section that is much higher than it is wide, a single keel that is not bordered by furrows, and moderately spaced, simple, slightly flexuous ribs. On the smallest specimens up to a diameter of about $27 \mathrm{~mm}$, the ribs trend radially or curve adapically on the flanks, become stronger ventrally, and terminate abruptly on the ventral margin (pl. 4, figs. 18-21). During further growth, as shown on the larger specimens, the ribs gradually become nearly radial, or incline slightly adorally on the flanks, and their ventral ends curve a little adorally.

These specimens closely resemble the paratypes of Melanhippites harbledownensis Crickmay (1928, pl. 4, figs. a-d) and likewise are associated with Entolium? semiplicatum (Hyatt) (equals E. balteatum Crickmay, 1928, pl. 4 figs. e-g). The ribbing on the Alaskan specimens is slightly sparser than on the holotype of Paltechioceras aplanatum Hyatt (Buckman, 1924, pl. 482) but nearly identical with that on the same species as figured by Getty (1973, p. 20, pl. 4, fig. 1a,b). The ribbing is likewise similar to that on Vermiceras bavaricum mexicanum Erben (1956, p. 207, pl. 36, figs. 507), which is assigned to Paltechioceras by Hallam (1965, p. 1493).

Figured specimens.-USNM 248000-248002.

Occurrences.-Talkeetna Formation in Talkeetna Mountains at USGS Mesozoic locs. 28661 and 28663; upper member of McCarthy Formation in the Wrangell Mountains at USGS Mesozoic locs. 30139 and 31174.

Subgenus ORTHECHIOCERAS Trueman and Williams, 1925 Paltechioceras (Orthechioceras?) sp.

Plate 3 , figures 11,12

One small specimen, consisting of five incomplete whorls, is characterized by highly evolute coiling, by a subquadrate whorl section that is slightly wider than high; by a carinate bisulcate venter whose sulci are fairly shallow; and by simple forwardly curved ribs that are rather closely spaced on the innermost whorls but become more widely spaced on the outermost whorl.

The specimen shows considerable resemblance to $P$. (O.) radiatum Trueman and Williams (1925, p. 724, pl. 2, fig. 9a,b; Getty, 1973, p. 23, pl. 5 figs. 1a,b, 2a,b), but its ribbing is denser on its four smallest whorls. It also shows some resemblance to the inner whorls of $P$. $(P$. elicitum Buckman (1924, pl. 483) but differs by having shallower sulci and by ribs which become more widely spaced adorally.
The specimen is assigned to the subgenus Orthechioceras rather than the subgenus Paltechioceras because of its fairly shallow sulci and because its ribs become widely spaced during growth. Its poor preservation, however, does not warrant a positive subgeneric determination.

Figured specimen.-USNM 248003.

Occurrence.-Unnamed beds in the Healy (A-6) quadrangle in Alaska Range at USGS Mesozoic loc. 31261.

\section{Family EODEROCERATIDAE Spath, 1929 Subfamily XIPHEROCERATINAE Spath, 1925 Genus CRUCILOBICERAS Buckman, 1920 \\ Crucilobiceras cf. C. crucilobatum Buckman \\ Plate 5 , figures 7,8}

One laterally crushed, fairly large external mold represents a highly evolute, fairly large bituberculate ammonite that is similar is appearance to Crucilobiceras and Microderoceras. Its outer two whorls bear rather weak, fairly sparse radial ribs that become stronger ventrally. The tubercles in the outer row occur near the ventral margin, are fairly prominent, and are round to slightly elongate spirally. The tubercles in the inner row occur at about the top of the lower third of the flanks, are fairly weak, are elongate radially, and appear to be a little stronger on the outermost whorl than on the next smaller whorl. The smallest whorls are much corroded.

The same species is probably represented by one small crushed mold that differs from the large specimen mainly by having more pronounced ribs and tubercles.

These specimens are similar in appearance to $C$. crucilobatum Buckman (1920, pl. 178) except for having more closely spaced ribs. Their assignment to Crucilobiceras rather than Microderoceras is based on the weakness of the inner row of tubercles and the rather high ventral position of the outer row of tubercles.

In England $C$. crucilobatum Buckman occurs in the lower part of the Echioceras raricostatum zone (Buckman, 1920, v. 3, pl. 178).

Figured specimens.-USNM 248004, 248005.

Occurrence.-Upper member of McCarthy Formation in the Chitina Valley of Wrangell Mountains at USGS Mesozoic loc. 14472 and probably at Mesozoic loc. 14030.

Crucilobiceras cf. C. densinodulum Buckman

Plate 7, figures 4, 5

cf. Crucilobiceras densinodulum Buckman, 1923, Yorkshire type ammonites, v. 5 , pl. 442.

cf. C. densinodulum Buckman. Dean, Donovan, and Howarth, 1961, p. 459 , pl. 67 , fig. 5 .

One worn specimen is characterized by highly evolute coiling; by a quadrate whorl section that is a little wider than high; and by very widely spaced flank ribs that incline slightly adorally, are swollen radially on the lower 
part of the flanks, weaken near the middle of the flanks, and terminate ventrally in prominent spirally elongate tubercles. These tubercles are well preserved at only one place.

This specimen differs from $C$. densinodulum Buckman by having weaker ribs near the middle of its flanks. $C$. densinodum (Quenstedt) as figured by Wright (1880, pl. 39 , figs. 6,$7 ; 1882$, pl. 50 , figs. 11,12 ) has much stronger ribbing and a higher whorl section. The species $C$. densinodulum occurs in the lower part of the Echioceras raricostatum zone (Dean and others, 1961, p. 459).

Figured specimen.-USNM 248006

Occurrence.-Talkeetna Formation in Talkeetna Mountains at USGS Mesozoic loc. 28661.

\section{Crucilobiceras cf. C. muticum (d'Orbigny)}

Plate 7, figures 6-10, 12-15

This species is represented by 17 laterally compressed specimens, of which 15 are from USGS Mesozoic loc. 28661. It is characterized by highly evolute coiling; a subquadrate whorl section that is a little higher than wide; and ribs that are strong, straight, rather sparse, incline forward on the flanks, become stronger ventrally, and terminate in fairly prominent tubercles on the ventral margin. The venter is gently rounded, smooth on the inner whorls, but marked on some outer whorls by weak swellings that arch gently forward from the tubercles. Most of the specimens have rather widely spaced ribs, as in C. muticum (d' Orbigny) (1844, p. 274, pl. 80).

C. muticum (d'Orbigny) in Europe is recorded from the Uptonia jamesoni Zone at the base of the Pliensbachian and questionably from the top of the underlying Echioceras raricostatum Zone (Bremer, 1965, p. 155; Mouterde and Ruget, 1970, p. 50; Geczy, 1976, p. 57).

The specimens herein compared with $C$. muticum (d'Orbigny) and C. submuticum (Oppel) are associated with echioceratid ammonites and hence must be of latest Sinemurian Age.

Figured specimens.-USNM 248007.

Occurrences.-Talkeetna Formation in Talkeetna Mountains at USGS Mesozoic locs. 28661, 28662, and 28663.

\section{Crucilobiceras cf. C. submuticum (Oppel)}

Plate 7, figures 1-3

Six specimens differ from those herein compared with C. muticum (d'Orbigny) by having finer and more closely spaced ribs as in C. submuticum (Oppel) (Quenstedt, 1885 , pl. 33, figs. 1, 9, 19, 23). As five of these specimens are associated with the specimens herein compared with C. muticum (d'Orbigny), they may all be variants of a single species.
C. submuticum (Oppel) in Europe is recorded from the Uptonia jamesoni zone at the base of the Pliensbachian (Arkell, 1956, pl. 128; Bremer, 1965, p. 156). Similar finely ribbed specimens from Hungary are recorded from the Tragophylloceras ibex Zone (Geczy, 1976, p. $58)$.

Figured specimens. $-248008,248009$.

Occurrences. - Talkeetna Formation in the Talkeetna Mountains at USGS Mesozoic locs. 6706 and 28661.

\section{Crucilobiceras cf. C. pacificum Frebold \\ Plate 8, figures 10-12, 15-17}

ef. Crucilobiceras pacificum Frebold, 1970, Canadian Jour. Earth Sci., v. 7, no. 2, p. 435-437, pl. 1, figs. 4-8; pl. 2, fig. 10.

One specimen, represented by external and internal molds, is nearly identical in whorl shape, ribbing, and tuberculation with the type specimens of Crucilobiceras pacificum Frebold. Its cross section is quadrate, slightly higher than wide, and is broadest at the ventrolateral border. Its venter is nearly flat and its flanks are slightly convex. Its ribs on the flanks are widely spaced, fairly strong, incline slightly forward, and terminate on the ventrolateral margin in nodes that are elongate spirally. Between successive strong ribs generally occur single weaker ribs, most of which fade out on the upper parts of the flanks. The surface of the venter is somewhat irregular but is not marked by ribs.

The identification of this specimen with Crucilobiceras pacificum Frebold is favored not only by these features but by the fact that it is likewise associated with fairly well preserved specimens of Tropidoceras actaeon (d'Orbigny), as in the Queen Charlotte Islands.

Figured specimens.-USNM 248010.

Occurrence.-Near top of upper member of the McCarthy Formation in the Wrangell Mountains at USGS Mesozoic loc. 28534.

\section{Subfamily COELOCERATIDAE Haug, 1910 Genus APODEROCERAS Buckman, 1921 \\ Apoderoceras cf. A. subtriangulare (Young and Bird) \\ Plate 8, figures 14, 18-23}

cf. Deroceras subtriangulare (Young and Bird). Buckman, 1913, Yorkshire type ammonites, v. 2, pl. 71A, B.

cf. Platypleuroceras? sp. indet. Frebold, 1970, Canadian Jour. Earth Sci., v. 7 , no. 2 , p. 439 , pl. 1 , fig. 3 ).

This species is represented by two specimens. It is characterized by evolute coiling; a wide, depressed whorl section that is widest near the venter; a venter that is slightly arched on the smallest preserved whorls, nearly flat on the largest whorls, and smooth or faintly striate; and straight simple ribs that begin low on the umbilical wall, incline slightly adorally on the flanks, and terminate in prominent nodes on the margin of the venter. The ribs exposed in the umbilicus of the innermost preserved whorls are sharp and fairly closely 
spaced, but at a diameter of about $25 \mathrm{~mm}$ they become much stronger and more widely spaced. The suture line is not preserved.

The largest specimen at a diameter of about $120 \mathrm{~mm}$ has a whorl height of about $30 \mathrm{~mm}$, a whorl thickness of $46 \mathrm{~mm}$, and an umbilical width of $68 \mathrm{~mm}$.

The largest preserved whorl of this species resembles that of the holotype of $A$. subtriangulare (Young and Bird) (Buckman, 1913, pl. 71A,B) from England but differs by having a flatter venter and more closely spaced ribs. The smallest whorls resemble those of Platypleuroceras? sp. indet. of Frebold (1970, pl. 1, fig. 3) from the McConnell Creek area in western British Columbia, except possibly for an abrupt change in strength of ribbing.

Figured specimens.-USNM 248011, 248012.

Occurrences.-Talkeetna Formation in the Talkeetna Mountains at USGS Mesozoic locs. 6697 and M6171.

\section{Family OXYNOTICERATIDAE Hyatt, 1875 \\ Genus FANNINOCERAS McLearn, 1930 \\ Fanninoceras kunae McLearn}

Plate 7, figure 11

Four immature specimens resemble $F$. kunae McLearn (1930, p. 5, pl. 2, fig. 4; 1932, pl. 9, figs. 1-6; Frebold, 1964b, pl. 9, fig. 4) in having a fairly wide umbilicus, a narrowly rounded venter, and moderately strong ribs that project forward on the flanks and become very weak on the venter.

Hypotype.-USNM 248013.

Occurrences. - Talkeetna Formation in the Talkeetna Mountains at USGS Mesozoic locs. 24107, 24108, and 27586.

\section{Fanninoceras cf. F. carlottense McLearn}

Twelve specimens resemble Fanninoceras carlottense McLearn (1932, p. 76, pl. 8, figs. 9, 10) in having a tiny umbilicus, a sharp venter, very weak, widely spaced ribs on small and intermediate-sized whorls, and nearly smooth outer whorls.

Occurrences. - Talkeetna Formation in the Talkeetna Mountains at USGS Mesozoic locs. 24108 and 29449.

\section{Family POLYMORPHITIDAE Haug, 1887 Genus UPTONIA Buckman, 1898 \\ Uptonia cf. U. dayiceroides Mouterde \\ Plate 9, figures 1-4, 8, 12-16}

cf. Uptonia dayiceroides Mouterde, 1951, Soc. Geol. Portugal Bol., v. 9 , no. 3 , p. 179 , pl. 1, figs. 4-6.

Uptonia cf. $U$. dayiceroides Mouterde. Frebold, 1970, Canadian Journal Earth Sci., v. 7, no. 2, p. 438, pl. 1, figs. 9a,b,c.

Eighty laterally crushed molds from the Wrangell Mountains are identical in coiling and density of ribbing with a specimen from the Queen Charlotte Islands that Frebold (1970, p. 438, pl. 1, figs. 9a,b) compared with Uptonia dayiceroides Mouterde.
On the small and intermediate-sized specimens from Alaska, the flank ribs are gently flexuous, become stronger ventrally, bear tubercles on the ventral margin, and then curve adorally. On the largest available specimens the flank ribs differ by not bearing tubercles. None of the specimens shows the middle part of the venter. Several associated specimens (pl. 9, figs. $2,14)$ that have slightly coarser and sparser ribbing are herein interpreted as a variant.

Figured specimens.-USNM 248014, 248015.

Occurrences. - Upper member of McCarthy Formation in the Wrangell Mountains at USGS Mesozoic locs. 28671-28673 and 28675.

Uptonia cf. U. jamesoni (J. de C. Sowerby)

Plate 9, figure 17

One laterally crushed ammonite from northern Alaska greatly resembles a specimen of Uptonia jamesoni (J. de C. Sowerby) from England (Wright, 1882, p. 352, pl. 51, figs. 1-3; Dean and others, 1961, pl. 68, figs. 3a,b) in its evolute coiling and in its ribbing. Its ribs are fairly strong, incline slightly forward on the flanks, incline strongly forward on the margins of the venter, become swollen ventrally, and become much stronger adorally. Sharp ventral tubercles are not evident. Most of the venter is not exposed.

Figured specimen.-USNM 248016.

Occurrence.-Unnamed shale in northern Alaska at USGS Mesozoic loc. 29774.

\section{Uptonia? sp. \\ Plate 9, figures 9-11}

Six small crushed ammonites from northern Alaska are nearly identical in coiling and ornamentation with the inner whorls of the fairly large ammonite described herein (pl. 9, fig. 17) as Uptonia cf. U. jamesoni (J. de C. Sowerby). The only difference consists of the presence of very small, sharp tubercles on some ribs at the ventral margin. Such close resemblances suggest that the small ammonites are probably immature forms of the same species as the large ammonite. Their assignment to $U p$ tonia rather than Crucilobiceras is favored by the forward curvature of their ribs on the highest parts of the flanks.

Figured specimens.-USNM 248017.

Occurrences.-Unnamed shale in northern Alaska at USGS Mesozoic locs. 29775, 29281, and 29282.

\section{Uptonia? sp. A}

Plate 8, figure 13

One fragmentary internal mold of a fairly large whorl bears ribbing similar to that on large specimens of Crucilobiceras and Uptonia. Assignment to Uptonia instead of Crucilobiceras is favored by the forward cur- 
vature of the ribs at the ventral margin and by the fact that the ribs are not distinctly tuberculate.

The specimen constitutes the only evidence for the presence of Lower Jurassic beds in the Yakutat district. Figured specimen.-USNM 248018.

Occurrence.-Float from unnamed beds in the Yakutat D-4 quadrangle, southeastern Alaska, at USGS Mesozoic loc. 29773.

\section{Uptonia? sp. B}

Plate 9, figures 5-7

Two small specimens have a compressed, fairly evolute shell and fairly sharp, simple ribs that incline slightly adorally on the flanks, become stronger ventrally, form pronounced chevrons on the venter, and do not bear ventral tubercles.

These features suggest that the specimens are immature forms of Uptonia. Such an assignment is suggested by their association with a fairly large, typical specimen of Apoderoceras, which genus in Europe is characteristic of the lower part of the Uptonia jamesoni zone of earliest Pliensbachian Age (Dean and others, 1961, p. 463).

Figured specimen.-248019.

Occurrence.-Talkeetna Formation in the Talkeetna Mountains at USGS Mesozoic loc. 6697.

\section{Genus TROPIDOCERAS Hyatt, 1867 \\ Tropidoceras actaeon (d'Orbigny) \\ Plate 8, figures 1-9 \\ (For synonymy see Frebold, 1970, p. 440)}

This species is represented in Alaska by four molds that are identical in appearance with the type specimens of $T$. actaeon (d'Orbigny) (1844, p. 232, pl. 61, figs. 1, 2) from France and with specimens of that species from the Queen Charlotte Islands illustrated by Frebold (1970, pl. 2, figs. 13a,b, 14a,b, 15a,b). The Alaskan specimens of the species have fairly evolute coiling. Their ribs are simple, moderately spaced, radial or slightly sigmoidal on the flanks, and they curve forward sharply on the ventral margins, where they fade out abruptly. On internal molds the venter ranges from narrowly rounded to fairly sharp and in places on some specimens bears a low blunt keel.

In Europe, this species is indicative of the lower to middle parts of the Tragophylloceras ibex zone of early Pliensbachian Age, according to Frebold (1970, p. 444, 445).

Hypotypes. -USNM 248020-248023.

Occurrence.-Near top of upper member of the McCarthy Formation in Wrangell Mountains at USGS Mesozoic loc. 28534.
Family AMALTHEIDAE Hyatt, 1867 Genus AMALTHEUS Montfort, 1808

Amaltheus margaritatus (Montfort)

Plate 10, figures 25, 26

(For synonymy and description see Howarth, 1958, London, Palaeontographical Soc., p. 13, 14 and Dagis, 1976, p. 7-9.)

This species was provisionally identified by Imlay in 1955 (p. 87, pl. 10, fig. 4) based on a specimen from a test well in the Point Barrow area. The specific identification was subsequently confirmed by Howarth (1958, p. xxvi). Hypotypes.-USNM 108768, 248024, 248025.

Occurrences.-Kingak Shale in northern Alaska in the South Barrow test well No. 3 at depths of 2,019 feet $(639 \mathrm{~m}), 2,111$ feet $(634 \mathrm{~m})$, and probably at 2,107 feet (641 m); in the Simpson test well No. 1 at depth of 5,677 feet $(1,730 \mathrm{~m})$; and in unnamed beds in the DeLong Mountains at USGS Mesozoic loc. M2441.

\section{Amaltheus stokesi (J. Sowerby)}

Plate 10, figures 27, 28

(For most of the synonomy see Howarth, 1958, London, Palaeontographical Soc., pt. 1, p. 3-6.)

Amaltheus cf. A. nudus (Quenstedt). Imlay, 1955, USGS Prof. Paper 274-D, p. 87 , pl. 10 , fig. 5 .

Amaltheus stokesi (J. Sowerby). Howarth, 1958, London, Palaeontographical Soc. pt. 2, p. xxvi.

This species has strong, slightly sigmoidal, radialtrending ribs that curve forward rather strongly high on the flanks, where they bifurcate or trifurcate and then merge with chevrons on the venter. It is distinguished from Amaltheus margaritatus (Montfort), according to Howarth $(1958$, p. 6), by having ventral chevrons that are larger, fewer, less forwardly inclined, and distinctly connected by twos or threes with the flank ribs instead of being connected only by striae or separated by a smooth band.

The presence of $A$. stokesi in Alaska was first recognized by Howarth (1958, p. xxvi) on the basis of an illustration published by Imlay (1955, pl. 10, fig. 5). Furthermore, Howarth identified $A$. cf. A. stokesi on the basis of another illustration by Imlay (1955, pl. 10, fig. 1). Other small specimens comparable with $A$. stokesi are illustrated on plate 10, figures 23 and 24 .

Hypotypes.-USNM 108769, 248026, 248027.

Occurrences.-Kingak Shale in northern Alaska in the South Barrow test well No. 3 at depths of 2,193 and 2,198 feet ( 668.4 and $670 \mathrm{~m}$ ); from unnamed beds cropping out in northern Alaska at USGS Mesozoic loc. 29165; and from Glenn Shale in east-central Alaska at Mesozoic loc. 29340.

Amaltheus cf. A. stokesi (J. Sowerby) occurs in the Kingak Shale in northern Alaska in the South Barrow test well No. 3 at the depth of 2,186 feet $(666.3 \mathrm{~m})$ and in outcrops at USGS Mesozoic loc. 30074. It occurs in the 
Talkeetna Formation in the Talkeetna Mountains in southern Alaska at USGS Mesozoic loc. 25941.

\section{Subgenus PSEUDOAMALTHEUS Frebold, 1922}

A. (Pseudoamaltheus) engelhardti (d'Orbigny)

This taxon is represented in northern Alaska by a fragment from the South Barrow test well No. 3 at the depth of 2090 feet $(637 \mathrm{~m})$. It was assigned by Imlay (1955, p. 87, pl. 10, fig. 3) to Amaltheus sp. and by Howarth (1958, p. xxvi) to $A$. (Pseudoamaltheus) engelhardti (d'Orbigny). Howarth's identification was based on the presence of spiral ribs on the upper part of the flanks and on the keel, and on the lack of radial ribbing. He noted that the species and subgenus in Europe ranges from the upper part of the Amaltheus margaritatus zone to the end of the Pliensbachian, or Pleuroceras spinatum zone, and is a descendant of Amaltheus margaritatus (Howarth, 1958, p. 21-23).

Hypotype.-USNM 108766.

\section{Family DACTYLIOCERATIDAE Hyatt, 1867 Genus PRODACTYLIOCERAS Spath \\ Prodactylioceras italicum italicum (Fucini) \\ Plate 10 , figure 3}

Coeloceras lorioli Bettoni, 1900, Schweizer. palaeont. Gesell. Abh., v. 27 , p. 76 , pl. 7 , fig. 12 .

Coeloceras italicum Meneghini in Fucini, 1900, Palaeontographica Italica, v. 7, p. 72, pl. 13, fig. 4.

Coeloceras italicum Meneghini in Fucini, 1905, Palaeontographica Italica, v. 11, p. 115, pl. 6, figs. 11, 12, 14.

Prodactylioceras italicum italicum (Fucini) in Fischer, 1971, Geologica et Palaeontologica, v. 5, p. 111, pl. 2, fig. 12.

Prodactylioceras (Aveyroniceras) italicum (Meneghini in Fucini).

Geczy, 1976, Akad. Kiado, Budapest, p. 145, pl. 25, figs. 8-9; pl. 26, figs. 1-4.

One laterally crushed specimen from Alaska is characterized by highly evolute coiling, by very fine, dense, simple, forwardly inclined ribs that become a little stronger ventrally, and by a few weak tubercles high on the flanks of the innermost whorls. Its appearance is identical with that of the finely ribbed subspecies, $P$. italicum italicum (Fucini) from Italy as described in Fischer (1971, p. 111). It has slightly finer ribbing than a specimen from eastern Oregon described as $P$. cf. $P$. italicum Meneghini (Imlay, 1968, p. C28, pl. 2, fig. 14). It differs from specimens in northern British Columbia and southern Yukon, described by Frebold (1964a, p. 10, pl. 3, figs. 2; 1970, p. 442, pl. 4, figs. 1, 2), by lacking forked ribs.

The association of the Alaskan specimen of Prodactylioceras with Uptonia shows that it occurs at or near the base of the Pliensbachian. Comparable finely ribbed specimens from southern Europe as listed herein are recorded from the zone of Tragophylloceras ibex near the base of the Pliensbachian (Fischer, 1971, p. 118, 123).
Figured specimen.-USNM 248030.

Occurrence.-Upper member of the McCarthy Formation in upper $100 \mathrm{ft}(30.5 \mathrm{~m})$, Wrangell Mountains, at USGS Mesozoic loc. 28671.

\section{Prodactylioceras cf. P. italicum fucini R. Fischer \\ Plate 10, figures 4,5}

Three crushed molds are characterized by highly evolute coiling; by high, narrow, moderately spaced nearly radial ribs that become slightly stronger ventrally and bifurcate rarely, low on the flanks; and by lacking tubercles, at least on their outer whorls. Their rib spacing resembles that on $P$. italicum fucini $R$. Fischer (1971, p. 111, pl. 2, figs. 8, 11) and is somewhat denser than in P. colubriforme (Bettoni) $(1900$, pl. 7 , fig. 10; Pinna, 1966, pl. 10, fig. 6; Fischer, 1971, pl. 2, fig. 10) from southern Europe. The resemblance of these Alaskan specimens to $P$. italicum fucini Fischer plus their association with Uptonia suggests an age as early as the zone of Uptonia jamesoni (Fischer, 1971, p. 118, 123) or as the lower part of the Tragophylloceras ibex zone.

Figured specimens.-USNM 248031, 248032.

Occurrences. - Upper member of McCarthy Formation on Wrangell Mountains at USGS Mesozoic locs. 28540, 28671 , and 28673.

\section{Genus DACTYLIOCERAS Hyatt, 1867 \\ Dactylioceras cf. D. commune (J. Sowerby) \\ Plate 11, figures 2, 3, 8}

Four laterally compressed specimens from the Talkeetna Mountains are characterized by evolute coiling and by sharp, fairly prominent, moderately spaced primary ribs which are inclined slightly forward. Most of the primary ribs pass at about two thirds of the height of the flanks into pairs of slightly weaker secondary ribs that arch gently forward on the venter. Some primary ribs remain simple, and some secondary ribs arise freely on the margins of the venter. Tiny tubercles occur at the furcation points.

These specimens show some resemblance to Dactylioceras crassiusculosum (Simpson) (Buckman, 1912, pl. 62; Fischer, 1966, p. 29, pl. 1, figs. 11; pl. 3, fig. 11; Pinna, 1966, p. 94, pl. 5, fig. 3; Sapunov, 1963, p. 120, pl. 2 , fig. $3 a, b)$, but they differ by having more closely spaced, weaker ribs that incline forward on the flanks instead of trending nearly radially. Compared with the lectotype of $D$. commune (Sowerby) (Buckman, 1927, pl. 707; Arkell, 1956, pl. 33, figs. 4a,b), they have denser and weaker ribs on their outermost preserved whorl. They do not differ greatly in ribbing, however, from $D$. crassibundum (Simpson), which Howarth (1962a, p. 115, pl. 16, fig. $7 \mathrm{a}, \mathrm{b})$ considered to be a synonym of $D$. commune (Sowerby). 
These specimens from the Talkeetna Mountains are nearly identical with most of the specimens from arctic Canada that were assigned to $D$. commune (Sowerby) by Frebold (1958, p. 2, pl. 1, figs. 1-7; 1960, p. 18, pl. 5, figs. $4,6)$ but have somewhat sparser ribbing than one specimen so assigned (Frebold, 1960, pl. 5, fig. 5).

Figured specimen.-USNM 248047.

Occurrence.-Talkeetna Formation in the Talkeetna Mountains at USGS Mesozoic locs. 24787 and 29198.

\section{Subgenus ORTHODACTYLITES Buckman, 1926}

Dactylioceras (Orthodactylites) kanense McLearn

Plate 11, figures 4, 5, 9

Dactylioceras kanense McLearn, 1930, Royal Soc. Canada Trans., 3d. ser., v. 24 , sec. 4 , p. 4 , pl. 1 , fig. 2

Dactylioceras kanense McLearn, 1932, Royal Soc. Canada Trans., 3d ser., v. 26 , sec. 4 , p. $59-62$, pl. 3 , fig. 5 , pl. 4 , figs. $1-7,9$ pl. 5 , figs. 6-9.

Dactylioceras kanense McLearn. Frebold, 1964b, Canada Geol. Survey Paper 63-4, pl. 7, figs. 1-4.

?Dactylioceras cf. D. kanense McLearn. Imlay, 1955, U.S. Geol. Survey Prof. Paper 274-D, p. D88, pl. 10. fig. 14.

This species is represented by four molds that are nearly identical in appearance with the holotype. The species is characterized by an evolute, compressed shell, by a narrowly rounded venter, and by fine threadlike ribs that incline gently forward on the flanks and arch forward on the venter. On the inner whorls, furcation of the ribs is fairly common. On the largest whorl, most of the primary ribs are simple and many ribs arise freely on the upper parts of the flanks. The species shows considerable resemblance to $D$. attenuatus (Simpson) (Buckman, 1926, pl. 655) but has fewer forked ribs on its outer whorl.

Hypotype.-USNM 248048.

Occurrence.-Talkeetna Formation in Talkeetna Mountains at USGS Mesozoic loc. 29198. Possibly represented in northern Alaska in the South Barrow test well No. 3 at the depth of 2,016 feet $(614.5 \mathrm{~m})$ (Imlay, 1955 , p. 82,88 ).

\section{Dactylioceras (Orthodactylites) cf. D. directum (Buckman) Plate 11, figure 6}

cf. Orthodactylites directum Buckman, 1926, Type ammonites, pl. 654. cf. Orthodactylites mitis Buckman, 1927, Type ammonites, pl. 738.

One specimen, whose outermost preserved whorl is crushed laterally, resembles the holotype specimen of $D$. (O.) directum Buckman in coiling and ribbing. It also resembles $D$. (O.) mitis Buckman, which Howarth (1973, p. 254, 255) considered to be a synonym of $D$. (O.) directum. As in those species, its ribs are fairly strong, nearly straight, rectiradiate, cross the venter nearly transversely and mostly bifurcate high on the flanks. Some ribs, however, remain simple.

Figured specimen.-USNM 248049.
Occurrence.-Kingak Shale in northern Alaska at USGS Mesozoic loc. 29163.

\section{Genus CATACOELOCERAS Buckman, 1923}

Catacoeloceras? sp. juv.

Plate 12, figure 6

Coeloceras aff. C. mucronatum (d'Orbigny). Imlay, 1955, U.S. Geol. Survey Prof. Paper 274-D, p. D88, pl. 12, figs. 12-14.

Catacoeloceras sp. "aff. mucronatum (d'Orbigny)". Howarth, 1958, London, Palaeontographical Soc. pt. 2, p. xxvi.

The assignment of this species to Catacoeloceras by Howarth was probably based on the generic definition published by Arkell and others (1957, p. L254). Later studies by Howarth (1962b, p. 407-410) showed, however, that most of the taxa listed in the treatise as synonyms of Catacoeloceras actually had different characteristics than the type species of Catacoeloceras, occurred in older beds just above the upper range of Amaltheus, and could appropriately be referred to Nodicoeloceras. He noted that Nodicoeloceras had small tubercles and looped ribs only on its innermost whorls. In contrast Catacoeloceras developed small ventrolateral tubercles on its inner and on some intermediate whorls but did not develop looped ribs.

The stratigraphic position of the Alaskan species above Amaltheus and below Dactylioceras (Imlay, 1955, p. $82 ; 1968$, p. C21) favors an assignment to Nodicoeloceras rather than to Catacoeloceras. Nonetheless the presence of fairly prominent tubercles and the lack of looped ribs are indicative of Catacoeloceras such as C. confectum Buckman (1923, pl. 413) and C. crassum (Young and Bird) (Buckman, 1918, pl. 119).

Figured specimen.-USNM 108758a-c.

Occurrence.-Kingak Shale in South Barrow test well No. 3 at depth of $2,063 \mathrm{ft}(629 \mathrm{~m})$.

\section{Family HILDOCERATIDAE Hyatt, 1867 Subfamily ARIETICERATINAE Howarth, 1955 Genus ARIETICERAS Seguenza, 1885 \\ Arieticeras cf. A. domarense (Meneghini) Plate 10, figures $1,2,6-15,22$}

This species is represented by 38 molds that match very well with specimens from eastern Oregon described previously under the same name (Imlay, 1968, p. C33, C34, pl. 4, figs. 9-12). Most of the molds also match certain specimens from northern British Columbia and southern Yukon that were assigned to Arieticeras $\mathrm{cf}$. $A$. algovianum (Oppel) by Frebold (1964a, p. 13, pl. 3, fig. 3a,b; pl. 5, figs. 2, 3). The species differs from the species herein called $A$. cf. $A$. algovianum (Oppel) by having a higher, more compressed whorl section and finer, more closely spaced ribs.

Figured specimens.-USNM 248033-248037. 
Occurrences.-Talkeetna Formation in Talkeetna Mountains at USGS Mesozoic locs. 24107, 27586 and 29450. Lubbe Creek Formation in the Wrangell Mountains at Mesozoic loc. 28531. Upper member of the McCarthy Formation in the Wrangell Mountains at USGS Mesozoic loc. 28688.

\section{Arieticeras cf. A. algovianum (Oppel) \\ Plate 10, figures 16-20}

This species is represented by three fragments that resemble certain specimens from eastern Oregon (Imlay, 1968, p. C34, pl. 4, figs. 1-8) and from northwestern British Columbia (Frebold, 1964a, pl. 3, figs. 4a, b, 5a, b; pl. 4, fig. 2) that were compared, or identified with $A$. algovianum (Oppel). These specimens are characterized by highly evolute coiling, by a low keel that is bordered by shallow furrows, and by high, narrow, widely spaced ribs that trend radially or slightly adapically on the flanks.

Figured specimens.-USNM 248038.

Occurrence.-Upper member of McCarthy Formation, in the Wrangell Mountains at USGS Mesozoic loc. 28688.

\section{Arieticeras sp.}

Plate 10, figure 21

On one small specimen the innermost whorls are smooth up to a diameter of about $6 \mathrm{~mm}$. The next outer whorl, whose ventral part is not preserved, bears fairly low, moderately spaced ribs that curve slightly backward on the flanks. The outermost whorl bears fairly strong, widely spaced ribs that curve backward strongly on the lower and middle parts of the flanks, curve forward strongly on their upper parts, and then fade out on the ventral margin. The venter bears a low keel that is bordered by very shallow furrows.

This specimen differs from those in Oregon and British Columbia that have been compared or identified with $A$. algovianum (Oppel) (Imlay, 1968, p. C34, pl. 4, figs. 1-8; Frebold, 1964a, p. 13, pl. 3, figs. 4, 5; pl. 4, fig. 2) by having sparser ribs that curve backward much more strongly on the flanks. Its rib plan shows more resemblance to that of Arieticeras retrorsicosta (Oppel) as in Quenstedt (1885, pl. 42, fig. 44) and $A$. cf. A. retrorsicosta (Oppel) in Monestier (1934, pl. 10, figs. 42, 43).

Figured specimen.-USNM 248039.

Occurrence.-Lubbe Creek Formation in the Wrangell Mountains at USGS Mesozoic loc. 28531.

\section{Genus LEPTALEOCERAS Buckman, 1918}

Leptaleoceras cf. L. pseudoradians (Reynes)

Plate 11, figures 12,13

Ammonites pseudoradians Reynes, 1868, Essai de géologie et de paléontologie aveyronnaises: Paris (Baillère), p. 91, pl. 1, figs. 4a-c.
Arieticeras pseudoradians (Reynes). Monestier, 1934, Soc. Géol. France Mém., new ser., v. 10, pt. 3 (Mém. 23), p. 63, pl. 8 figs. 66 and 68.

Leptaleoceras pseudoradians (Reynes). Howarth, 1957, Geol. Soc. London Quart. Jour., v. 113, p. 198, pl. 17, figs. 1, 2.

Leptaleoceras pseudoradians (Reynes). Frebold, 1964a, Canada Geol. Survey Bull. 116, p. 15, pl. 4, figs. 5-7, pl. 5, figs. 4, 5 .

This species is represented by one fairly well preserved specimen that retains some shell material and is mostly septate. Its characteristics are identical with those of specimens from the southern Yukon and British Columbia as illustrated and described by Frebold.

Hypotype.-USNM 248040.

Occurrence.-Talkeetna Formation in Talkeetna Mountains at USGS Mesozoic loc. 29450.

Genus FONTANELLICERAS Fucini, 1931

Fontanelliceras cf. F. fontanellense (Gemmellaro)

Plate 11, figures $17-23$

(For synonymy see Imlay, 1968, U.S. Geol. Survey Prof. Paper 593-C, p. C36.)

cf. Fontanelliceras fontanellense (Gemmellaro). Guex, 1973, Eclogae geol. Helvetiae, v. 66 , p. 507 , pl. 6 , fig. 2 .

The genus Fontanelliceras is represented by five specimens. The largest specimen (pl. 11, figs. 17, 18, 23) has highly evolute coiling; a tricarinate bisulcate venter; and high, straight, rursiradiate ribs that become stronger ventrally and bend forward slightly before terminating on the ventral margin. The smaller specimens have been crushed laterally but resemble the larger in most respects. They differ by having ribs that are sharper, more widely spaced, and nearly radially arranged. The venter, which is well exposed on the largest specimen, bears a prominent keel bordered by furrows that in turn are bordered by weak ridges.

At a diameter of $59 \mathrm{~mm}$, the largest specimen has a whorl height of $14 \mathrm{~mm}$, a whorl thickness of $11 \mathrm{~mm}$, and an umbilical width of $35 \mathrm{~mm}$. Its suture line is not exposed.

Figured specimens.-USNM 248041-248043.

Occurrences. - Talkeetna Formation in the Talkeetna Mountains at USGS Mesozoic locs. 25941, 27586, and 29450.

\section{Subfamily HARPOCERATINAE Neumayr, 1875 Genus HARPOCERAS Waagen, 1869 \\ Harpoceras cf. H. exaratum (Young and Bird)}

Plate 11, figure 11

cf. Harpoceras exaratum (Young and Bird). Wright, 1882, London, Palaeontographical Soc. pl. 62, figs. 1-3.

Harpoceras cf. H. exaratum (Young and Bird). Imlay, 1955, U.S. Geol. Survey Prof. Paper 274-D, p. 88, pl. 11, figs. 12, 13, 15.

Harpoceras cf. H. exaratum (Young and Bird). Frebold and Little, 1962, Canada Geol. Survey Bull. 81, p. 17, 18, pl. 2, figs. 1-9, pl. 3, fig. 5 .

Harpoceras ef. H. exaratum (Young and Bird). Frebold, 1964 a, Canada Geol. Survey Bull. 116, p. 16, pl. 6, figs. 2-4 (not 1 and 5). 
Harpoceras cf. H. exaratum (Young and Bird). Frebold, 1957, Canada Geol. Survey Mem. 287, p. 47, pl. 17, fig. 1; pl. 18, figs. 2, 3 ,

This species is represented by five fragmentary specimens from northern Alaska and one from the Talkeetna Mountains in southern Alaska. Their features match very well with those on specimens from western and Arctic Canada as described and illustrated by Frebold in the papers listed above.

Figured specimen.-USNM 248044.

Occurrences.-Kingak Shale in northern Alaska at USGS Mesozoic localities 22081, 29159, 29160, 29161, and 29776. Talkeetna Formation in Talkeetna Mountains at Mesozoic loc. 29198.

\section{Genus PROTOGRAMMOCERAS Spath, 1913 \\ Protogrammoceras cf. P. paltum (Buckman) \\ Plate 12, figures 11, 12}

cf. Paltarpites paltus Buckman, 1922, Type ammonites, v. 4, pl. 362a and $1923, \mathrm{pl}$. $362 \mathrm{~b}$.

Harpoceras cf. H. exaratum (Young and Bird). Frebold, 1964a, Canada Geol. Survey Bull. 116, pl. 6, figs. 1, 5 (not 2-4).

cf. Paltarpites paltus Buckman. Frebold, 1970, Canadian Jour. Earth Sci., v. 7, no. 2, p. 443, pl. 4, figs. 5-7.

Three fragmentary specimens from Alaska bear ribbing similar to that on Protogrammoceras paltum (Buckman). They differ from $P$. argutum (Buckman) by having fewer ribs that are broader and flat topped. Nonetheless, as discussed by Frebold (1970, p. 443), the ribs during growth on some specimens of $P$. paltum vary considerably in strength from fine to coarse to fine, or even to striae. Consequently fragments of whorls bearing only striae, or only very fine ribs, cannot reliably be assigned to either species.

The assignment of both species to Protogrammoceras Spath rather than to Paltarpites Buckman, is based on examination of the type specimens by Howarth (1973, p. 265).

Figured specimen.-USNM 248045.

Occurrences.-Talkeetna Formation in the Talkeetna Mountains at USGS Mesozoic locs. 24108, 25941, and 29449.

\section{Protogrammoceras cf. P. argutum (Buckman)}

Plate 11, figure 14

cf. Argutarpites argutus Buckman, 1923, Type ammonites, v. 4, pl. 363.

Paltarpites cf. $P$. argutus Buckman. Imlay, 1968, U.S. Geol. Survey Prof. Paper 593-C, p. C37, pl. 5, figs. 20-22, 24, 25.

cf. Paltarpites argutus (Buckman). Frebold, 1970, Canadian Jour.

Earth Sci., v. 7, no. 2, p. 444, pl. 4, figs. 8-10.

This species is represented by two fragments, that are characterized by very fine falcoid ribs and striae and by a rounded umbilical edge. It could be interpreted as a finely ribbed variant of $P$. paltum (Buckman), which species is associated with $P$. argutum (Buckman) in western Canada (Frebold, 1970, p. 444).
Figured specimen.-USNM 248046.

Occurrences.-Talkeetna Formation in Talkeetna Mountains at USGS Mesozoic locs. 24108 and 25941.

\section{Genus ELEGANTICERAS Buckman, 1913}

Eleganticeras sp. juv.

Plate 11, figures $10,15,16$

This species is represented by 12 small internal and external molds. They have a moderately compressed planulate form; a low vertical umbilical wall that rounds fairly abruptly into somewhat flattened flanks; a rather sharp keel on the venter; and an incomplete body chamber that occupies at least half a whorl. The innermost whorls are smooth. Weak, widely spaced ribs appear at a diameter of 7 to $9 \mathrm{~mm}$ and gradually become stronger during growth. The largest preserved septate whorl and the adapical end of the nonseptate whorl bear low falcoid ribs that are faint on the lower third of the flanks, become stronger ventrally, and extend almost to the keel. Such ribs are replaced rather abruptly on the adoral half of the largest preserved whorls by much weaker falcoid ribs and then by striae that are apparent only where some of the shell is preserved. The outermost whorl embraces about half of the preceding whorl.

In shape, coiling, and ribbing, this species greatly resembles the holotype of Elegantuliceras elegantulum (Young and Bird) (Buckman, 1914, pl. 93). It differs by being only half as large and by its umbilical wall being vertical instead of concave.

Figured specimen.-USNM 248050.

Occurrence.-Kingak Shale in northern Alaska at USGS Mesozoic loc. 29776.

\section{Genus PSEUDOLIOCERAS Buckman, 1913}

Pseudolioceras cf. P. compactile (Simpson)

(See Imlay, 1955, U.S. Geol. Survey Prof. Paper 274-D, p. 89, pl. 12, figs. 17, 18, 21.)

Occurrence.-Kingak Shale at USGS Mesozoic loc. 23772.

Pseudolioceras cf. P. lythense (Young and Bird)

(See Imlay, 1955, U.S. Geol. Survey Prof. Paper 274-D, p. D89, pl. 12, fig. 20.)

Occurrence.-Kingak Shale at USGS Mesozoic loc. 23772.

Pseudolioceras sp.

Plate 11, figure 1; Plate 12, figure 13

Two laterally compressed specimens from southern Alaska resemble $P$. maclintocki (Haughton) from arctic Canada and arctic Alaska (Frebold, 1964b, pl. 10, figs. 4-8, 12; Imlay, 1976, pl. 1, figs. 1-5, 7.) They appear to have a smaller umbilicus and sparser, weaker ribbing, but their preservation is not sufficient for a positive specific determination. 
Figured specimens.-USNM 248051, 248052.

Occurrences.-Talkeetna Formation in Talkeetna Mountains at USGS Mesozoic loc. 25317; Kialagvik Formation at Puale Bay on the Alaska Peninsula at USGS Mesozoic loc. 19804.

\section{Subfamily GRAMMOCERATINAE Buckman, 1904 Genus GRAMMOCERAS Hyatt, 1867 \\ Grammoceras sp. \\ Plate 11, figure 7}

One laterally crushed ammonite is characterized by evolute coiling by simple, strong, gently sigmoid ribs, and by a low keel as in Grammoceras. It has slightly coarser ribbing than does the ammonite from the southern Yukon described by Frebold (1964a, p. 17, pl. 7, figs. 1, 2) as G. aff. G. fallaciosum (Bayle).

Figured specimen.-USNM 248053.

Occurrence.-Talkeetna Formation, upper part, in the Talkeetna Mountains at USGS Mesozoic loc. 25939.

\section{Family HAMMATOCERATIDAE Buckman, 1887 Genus PHYMATOCERAS Hyatt, 1867 \\ Phymatoceras? sp. \\ Plate 12, figures 14, 16-19}

The genus is possibly represented by three specimens. The smallest specimen (pl. 12, fig. 18) bears twinned ribs and a carinate venter similar to that on Phymatoceras binodata (Buckman) (1898, Supplement, p. XVI, pl. 1, figs. 11, 12). In each twin the adapical rib is the stronger and is marked near the umbilical edge by a swelling. All ribs curve forward on the venter but are separated from the keel by a smooth area.

The next larger specimen is septate and bears alternating long and short ribs. The long ribs begin on the umbilical wall and are slightly swollen on the umbilical margin. The short ribs begin at or a little above the middle of the flanks. All ribs incline forward on the flanks and venter, become stronger ventrally, become swollen on the venter, and then terminate abruptly before reaching the keel.

The largest specimen is a nonseptate fragment that shows the venter and part of the flank of an adult whorl. It bears broad coarse ribs that are wider than the interspaces, that curve forward slightly on the flanks and more strongly on the venter, and that then terminate abruptly before reaching a rather low keel. The ventral aspect of this specimen is similar to that of $P$. tumefacta (Buckman) (1898, Supplement, p. XIX, pl. 1, figs. 7-10). Figured specimens.-USNM 248054-248056.

Occurrences.-Talkeetna Formation, upper part, in the Talkeetna Mountains at USGS Mesozoic locs. 25317 and 25342.

\section{Genus BRODIEIA Buckman, 1898}

Brodieia cf. B. tenuicostata var. nodosa (Jaworski) Plate 12, figure 8

cf. Hildoceras (Brodiceras) tenuicostatum var. nodosa Jaworski, 1926, Actas Acad. Nac. Cienc. Cordoba, v. 9, p. 245, pl. 4, fig. 8a,b.

One laterally crushed ammonite consists mostly of an incomplete body chamber that occupies at least threefourths of a whorl and embraces about two-thirds of the preceding whorl. The venter bears a single low keel. The ribs on the outer whorl are moderately strong, widely spaced, gently falcate, and terminate ventrally near the keel. The primary ribs begin low on the umbilical wall, are fairly strong near the umbilicus, weaken a little near the middle of the flanks, and are strongest on the margin of the venter. Some rib furcation occurs at various heights ranging from the umbilical margin to a little above the middle of the flanks. Other ribs arise freely near or above the middle of the flanks. All ribs are essentially of the same strength on the venter. The ribbing on the penultimate whorl as revealed in the umbilicus is also fairly strong.

This ammonite from Alaska bear ribbing almost identical with that on Brodieia tenuicostata var. nodosa (Jaworski) (1926, pl. 4, fig. 8a) from Argentina, but it differs by being a little more involute. In that respect it resembles the typical forms of $B$. tenuicostata (Jaworski) (1926, pl. 4, figs. 1-4, 6, 7) but differs by having slightly coarser and sparser ribbing. Overall the resemblances are remarkable.

Figured specimen.-USNM 248057.

Occurrence.-Talkeetna Formation in Talkeetna Mountains at USGS Mesozoic loc. 25940.

\section{Genus HAUGIA Buckman, 1888 \\ Haugia cf. H. grandis Buckman \\ Plate 12, figures $4,10,15$}

cf. Haugia grandis Buckman, 1898, Supplement, p. XXVI, pl. s, fig. $11 ; 1888$, pl. 23 , figs. 14,$15 ; 1889$, pl. 24 , pl. 25 , fig. 1

Ten specimens belong to a fairly involute species that has sharp, moderately spaced, flexuous ribs. The largest specimen is similar in size, involution, and ornamentation to a small specimen of $H$. grandis Buckman (1888, pl. 23, figs. 14, 15), but its tubercles are smaller and its ribs arch forward more strongly on its ventral margin. H. dumortieri Buckman (1888, pl. 23, figs. 16, 17) has fewer tubercles and ribs at a comparable size.

Figured specimen.-USNM 248058.

Occurrences.-Talkeetna Formation in the Talkeetna Mountains at USGS Mesozoic locs. 24111 and 24114; Kialagvik Formation at Puale Bay on the Alaska Peninsula at Mesozoic loc. 19804. 
Haugia cf. $H$. variabilis (d'Orbigny)

Plate 12, figures 1, 2, 5

cf. Ammonites variabilis d'Orbigny, 1845, Paléontologie française, Terrains Jurassiques, v. 1, Cephalopods, p. 350, p. 113, figs. 1 and 2 only.

cf. Harpoceras variabile (d'Orbigny). Wright, 1882, London,

Palaeontographical Soc., p. 455 , pl. 67 , figs. 5, 6 only.

cf. Haugia aff. H. variabilis (d'Orbigny). Buckman, 1890, London,

Palaeontographical Soc., p. 146, pl. 25, fig. 2.

This species is represented by nine crushed molds. It has fairly evolute coiling. The ornamentation on its inner whorls consists of umbilical tubercles, of sharp, nearly radially trending ribs that arise mostly by two's and three's from the tubercles, and of a few ribs that arise freely between the tubercles. During subsequent growth, both tubercles and ribs gradually become stronger and more widely spaced. The ribs become gently flexuous on the flanks, arch forward slightly on the venter, and arise mainly in pairs from prominent conical tubercles. The characteristics of the adult body chamber are unknown.

The Alaskan species may be within the range of variation of $H$. variabilis (d'Orbigny), but it appears to be more evolute and to develop sparser ribbing. In these respects it shows more resemblance to $H$. aff. $H$. japonica (Neumayr) as illustrated by Matsumoto and Ono (1947, pl. 2, figs. 5). It has much coarser ornamentation than the holotype of $H$. japonica (Neumayr) (Kobayashi, 1935, pl. 12, figs. 3, 4).

Figured specimens.-USNM 248059.

Occurrences.-Talkeetna Formation in the Talkeetna Mountains at USGS Mesozoic locs. 25318 and 27508.

\section{Haugia cf. H. compressa Buckman}

Plate 12, figures $3,7,9$

cf. Haugia compressa Buckman, 1898, London, Palaeontographical Soc., Supplement, p. XXVII, pl. 2, figs. 8-10.

Nine molds represent a fairly involute and densely ribbed species. The largest specimen at a diameter of 66 $\mathrm{mm}$ bears about 21 umbilical tubercles and about 65 gently flexuous ribs that arise in two's and three's from the tubercles. Many ribs arise freely along the zone of tuberculation.

This species closely resembles $H$. compressa Buckman in involution and in density of ribibng but at a comparable size has slightly finer ribbing and smaller tubercles than the holotype.

Figured specimens.-USNM 248060, 248061.

Occurrences.-Talkeetna Formation in the Talkeetna Mountains at USGS Mesozoic loc. 24114; basal part of Kialagvik Formation at Puale Bay on Alaska Peninsula at Mesozoic loc. 19804.

\section{REFERENCES CITED}

Arkell, W. J., 1956, Jurassic geology of the world: London, Oliver and Boyd, 806 p., 46 pls., 102 figs.

Arkell, W. J., and others, 1957, Treatise on invertebrate paleontology, R. C. Moore, ed.; pt. L, Mollusca 4-Cephalopoda, Ammonoidea: New York, Geol. Soc. America, and Lawrence, Univ. Kansas Press, 490 p.

Bettoni, Andrea, 1900, Fossili Domeriani della Provincia di Brescia: Schweizer. palaeont. Gesell. Abh., v. 27, p. 1-88, pls. 1-9.

Bremer, Heinrich, 1965, Zur Ammoniten Fauna und Stratigraphie des unteren Lias (Sinemurium bis Carixium) in der Umgebung von Ankara (Turkei): Neues Jahrbuch Geologie Paläontologie Abh., v. 122 , no. 2 , p. $127-221$, pls. $12-16,12$ figs.

Brosgé, W. P., and Reiser, H. N., 1964, Geologic map and section of the Chandalar quadrangle, Alaska: U.S. Geol. Survey Misc. Geol. Inv. Map I-375.

1969, Preliminary geologic map of the Coleen quadrangle, Alaska: U.S. Geol. Survey open-file report, scale 1:250,000.

Buckman, S. S., 1887-1907, Monograph of the ammonites of the Inferior Oolite Series: London, Palaeontographical Soc., 456 p., 103 pls. (1887-1894); Supplement, 262 p., 24 pls. (1893-1907).

-1909-1930, Yorkshire type ammonites, v. 1 and 2 (1909-1919); Type ammonites, v. 3-7 (1920-1930), ed. A. M. Davies (1930).

Crickmay, C. H., 1928, The stratigraphy of Parson Bay, British Columbia: California Univ. Pub. Dept. Geol. Sci., Bull., v. 18, no. 2, p. $51-70,4$ pls., 1 map.

1933, Some of Alpheus Hyatt's unfigured types from the Jurassic of California: U.S. Geol. Survey Prof. Paper 175-B, p. 51-58, pl. 14-18.

Dagis, A. A., 1976, [Late Pliensbachian ammonites (Amaltheidae) of north Siberia]: Novosibirsk, Publishing House Nauk USSR, Siberian Branch, p. 5-60, pls. 1-17, 8 figs. (In Russian)

Dean, W. T., Donovan, D. T., and Howarth, M. K., 1961, The Liassic ammonite zones and subzones of the north-west European province: British Mus. (Nat. History) Bull., v. 4, no. 10, p. 437-505, pls. $63-75$.

Detterman, R. L., Reiser, H. N., Brosgé, W. P., and Dutro, J. T., Jr. 1975, Post-Carboniferous stratigraphy, northeastern Alaska: U.S. Geol. Survey Prof. Paper. 886, 46 p., 14 figs.

Donovan, D. T., 1952, The ammonites of the Blue Lias of the Bristol district: Annals and Mag. Nat. History, ser. 12, v. 5, p. 629-655, 717-752, pls. 22-23, 27-29.

-1955, Revision des espèces décrites dans la Monographie des Ammonites (Lias inferieur) de P. Reynes: Soc. géol. France, Mém., new ser., v. 73, p. 1-45, 2 pls.

Donovan, D. T., and Forsey, G. F., 1973, Systematics of lower Liassic Ammonitina: Kansas Univ. Paleont. Contr., Paper 64, 18 p., 4 pls.

Erben, H. N., 1956, El Jurasico Inferior de Mexico y sus amonitas: Internat. Geol. Cong., 20th, Mexico, D. F., 1956, 393 p., 41 pls., 5 maps.

Fischer, Rudolf, 1966, Die Dactylioceratidae (Ammonoidea) der Kammerker (Nordtirol) und die zonengliederung des Alpinen Toarcian: Bayerische Akad. Wiss. Math.-Naturw. Kl., Abt. Abh., new ser., No. 126, p. 1-83, pls. 1-6, 4 figs.

1971, Die Coeloceratinen (Ammonoidea) des Monte Cetona (Prov. Siena)-Paläontologie, Geologie und Stratigraphie eines Profils im Mediterranen Carizium und Lotharingium: Geologica et Palaeontologica, v. 5, p. 93-129, 2 pls., 12 figs.

Frebold, Hans, 1951, Ammonite fauna and stratigraphy of the lower Lias in Tyaughton Lake map-area, British Columbia: Canada Geol. Survey Bull. 18, p. 1-14, pls. 1-14, 16-18.

1957, The Jurassic Fernie group in the Canadian Rocky Mountains and foothills: Canada Geol. Survey Mem. 287, 197 p., 44 pls., 5 figs. 
1958, Fauna, age and correlation of the Jurassic rocks of Prince Patrick Island: Canada Geol. Survey Bull. 49, 32 p., pls. 1-18, 2 figs.

1960, The Jurassic faunas of the Canadian Arctic-Lower Jurassic and lowermost Middle Jurassic ammonites: Canada Geol. Survey Bull. 59, 33 p., 15 pls., 8 figs.

1964a, Lower Jurassic and Bajocian ammonoid faunas of northwestern British Columbia and southern Yukon: Canada Geol. Survey Bull. 116, 31 p., 8 pls., 1 fig.

-1964b, Illustrations of Canadian fossils-Jurassic of western and arctic Canada: Canada Geol. Survey Paper 63-4, 106 p., 51 pls.

1967a, Position of the Lower Jurassic genus Fanninoceras McLearn and the age of the Maude Formation on Queen Charlotte Islands: Canadian Jour. Earth Sci., v. 4, no. 6, p. 1145-1149, 1 pl.

1967b, Hettangian ammonite faunas of the Taseko Lakes area, British Columbia: Canada Geol. Survey Bull. 158, 35, p., 9 pls. 6 figs.

1970, Pliensbachian ammonoids from British Columbia and southern Yukon: Canadian Jour. Earth Sci., v. 7, no. 2, p. 435-456, pls. 1-4.

Frebold, Hans, and Little, H. W., 1962, Paleontology, stratigraphy, and structure of the Jurassic rocks in Salmo map-area, British Columbia: Canada Geol. Survey Bull. 81, 31 p., 5 pls., 1 text-fig.

Frebold, Hans, and Poulton, T. P., 1977, Hettangian (Lower Jurassic) rocks and faunas, northern Yukon Territory: Canadian Jour. Earth Sci., v. 14, no. 1, p. 89-101, 2 pls., 3 figs.

Fucini, Alberto, 1900, Ammoniti del lias medio dell Appennino centrale: Palaeontographica Italica, v. 6, p. 17-78, pls. 7-13.

-1905 Cefalopoda liassici del Monte di Cetona: Palaeontographica Italica, v. 11, p. 93-146, pls. 3-11.

Geczy, Barnabas, 1976, Les ammonitines du Cariexien de la montagne du Bakony: Budapest, Akademiai Kiado, 223 p., 39 pls., 138 figs.

Getty, T. A., 1973, A revision of the generic classification of the family Echioceratidae (Cephalopoda, ammonoidea) (Lower Jurassic): Kansas Univ. Paleont. Contr. Paper 63, p. 1-32, 5 pls., 4 figs.

Grantz, Arthur, Thomas, Herman, Stern, T. W., and Sheffey, N. B. 1963, Potassium-argon and lead-alpha ages for stratigraphically bracketed plutonic rocks in the Talkeetna Mountains, Alaska: U.S. Geol. Survey Prof. Paper 475-B, p. B56-B59.

Guerin-Franiatte, S., 1966, Ammonites du Lias Inferieur de FrancePsilocerataceae, Arietitidae: Paris, Centre natl. recherche sci., 2 v., 231 pls., 190 figs., 10 maps.

Guex, Jean, 1973, Aperçu biostratigraphie sur le Toarcian inferieur du Moyen-Atlas marocain et discussion sur la zonation de ce sousétage dans les séries méditerranéennes: Eclogae geol. Helvetiae, v. 66 , no. 3 , p. $493-523,15$ pls., 4 figs.

Guex, Jean and Taylor, David, 1976, La limite Hettangian-Sinemurian des Prealpes romandes au Nevada: Eclogae geol. Helvetiae, v. 69, no. 2, p. 521-526.

Hallam, Anthony, 1965, Observations on marine Lower Jurassic stratigraphy of North America, with special reference to United States: Am. Assoc. Petroleum Geologists Bull., v. 49, p. 1481-1501, 5 figs.

Howarth, M. K., 1957, The Middle Lias of the Dorset Coast: Geol. Soc. London Quart. Jour., v. 113, p. 185-203, pl. 17

1958, A monograph of the ammonites of the Liassic family Amaltheidae in Britain: London Palaeontographical Soc., pt. 1, p. i-xiv, 1-26, pls. 1-4; pt. 2, p. xv-xxxvii, 27-53, pls. 5-10.

1962a, The Yorkshire type ammonites and nautiloids of Young and Bird, Phillips, and Martin Simpson: Palaeontology, v. 5, pt. 1, p. $93-136$, pls. $13-19$.

1962b, The Jet Rock Series and the Alum Shale Series of the Yorkshire Coast: Yorkshire Geol. Soc. Proc., v. 33, pt. 4, p. 381-422, pls. 24-28.
1973, The stratigraphy and ammonite fauna of the upper Liassic Grey Shales of the Yorkshire Coast: British Mus. (Natural History) Bull., v. 24, no. 4, p. 237-277, pls. 1-9, 6 figs.

Imlay, R. W., 1955, Characteristic Jurassic mollusks from northern Alaska: U.S. Geol. Survey Prof. Paper 274-D, p. 69-96, pls. 8-13, 1 fig., 4 tables.

1967, The Mesozoic pelecypods Otapiria Marwick and Lupherella Imlay, new genus, in the United States: U.S. Geol. Survey Prof. Paper 573-B, p. B1-B11, pls. 1, 2, 2 figs.

1968, Lower Jurassic (Pliensbachian and Toarcian) ammonites from eastern Oregon and California: U.S. Geol. Survey Prof. Paper 593-C, p. C1-C51, 9 pls., 8 figs.

1976, Middle Jurassic (Bajocian and Bathonian) ammonites from northern Alaska: U.S. Geol. Survey Prof. Paper 854, 22 p., 4 pls. 5 figs.

Imlay, R. W., and Detterman, R. L., 1973, Jurassic paleobiogeography of Alaska: U.S. Geol. Survey Prof. Paper 801, 34 p., 15 figs.

1977, Some Lower and Middle Jurassic beds in Puale BayAlinchak Bay area, Alaska: Am. Assoc. Petroleum Geologists Bull., v. 61, p. 607-611, 2 figs.

Jaworski, Erich, 1926, La fauna del Lias y Dogger de la Cordillera Argentina en la parte meridional de la Provincia de Mendoza: Acad. Nac. Cienc. Cordoba Actas, v. 9, nos. 3, 4, p. 139-319, pls. $1-4$.

Jones, D. L., Silberling, N. J., Csejtey, Bela, Nelson, W. T., and Blome, C. C., 1980, Age and structural significance of ophiolite and adjoining rocks in the upper Chulitna district, south-central Alaska: U.S. Geol. Survey Prof. Paper 1121-A, 21 p., 12 pls.

Kelley, J. S., 1978, Lithofacies in Lower Jurassic volcaniclastic rocks adjacent to lower Cook Inlet, southwestern Kenai Peninsula, Alaska: Am. Assoc. Petroleum Geologists Bull., v. 62, no. 11, p. 2356.

Kobayashi, Teiichi, 1935, Contribution to the Jurassic Torinosu series of Japan: Japanese Jour. Geology and Geography, v. 12, nos. 3-4, p. $59-91$, pls. 12,13 .

Lange, Warner, 1941, Die Ammoniten fauna der Psiloceras-stufe Norddeutschlands: Palaeontographica (Stuttgart), v. 93, Abt. A, 192 p., 20 pls.

1951, Die Schlotheimiinae aus dem Lias alpha Norddeutchlands: Palaeontographica (Stuttgart), v. 100, Abt. A, p. 1-128, 20 pls.

MacKevett, E. M., Jr., 1969, Three newly named Jurassic formations in the McCarthy C-5 quadrangle, Alaska: U.S. Geol. Survey Bull. 1274A, p. 35-49, figs. 3-8.

-1970 , Geologic map of the McCarthy C-4 quadrangle, Alaska: U.S. Geol. Survey Geol. Quad. Map GQ-844.

1971, Stratigraphy and general geology of the McCarthy C-5 quadrangle, Alaska: U.S. Geol. Survey Bull. 1323, 35 p., 3 figs.

Martin, G. C., 1926, The Mesozoic stratigraphy of Alaska: U.S. Geol. Survey Bull. 776, 493 p., 13 figs.

Matsumoto, Tatsuro, and Ono, Akira, 1947, A biostratigraphic study of the Toyora group, with special reference to ammonites: Kyushu Univ. Fac. Sci., Sci. Repts. Geology, v. 2, no. 1, p. 20-33, pls. 1, 2.

McLearn, F. H., 1930, Notes on some Canadian Mesozoic faunas: Roayl Soc. Canada Trans., ser. 3, v. 24, sec. 4, p. 1-8, 2 pls.

-1932, Contributions to the stratigraphy and paleontology of Skidgate Inlet, Queen Charlotte Islands, British Columbia: Royal. Soc. Canada Trans., ser. 3, v. 26, sec. 4, p. 51-80, pls. 1-10.

Monestier, Joseph, 1934, Ammonites du Domerien de la region sud-est de l'Aveyron et de quelques regions de las Lozere à l'exclusion des Amaltheides: Soc. Géol. France Mém., new ser., v. 10, pt. 3 (Mém. 23), 102 p., $111 \mathrm{pls}$.

Mouterde, Rene, 1951, Ammonites du Lias Portugais: Soc. géol. Portugal Bol., v. 9, no. 3, p. 175-188, 2 pls., 5 figs. 
Mouterde, Rene, and Ruget, Ch., 1970, Le Lias moyen de Sao Pedro de Muel, 1, Cephalopodes: Portugal Com. Serv. Géol., v. 54, p. 39-74, pls. 1-8.

Neumayr, Melchior, 1879, Zur Kenntnis der Fauna des unteren Lias in den Nordalpen: Geol. Reichsanst. Wien Abh., v. 7, pt. 5, 46 p., 7 pls.

Orbigny, Alcide d', 1842-1851, Paléontologie française-Terrains Jurassiques, v. 1, Cephalopods: Paris, 642 p., 234 pls. (For details on dates see Geol. Mag.,new ser.v. 6, p. 223-225. Davies Sherborn 1899.)

Pinna, Giovanni, 1966, Ammoniti del Lias Superiore (Toarcian) dell'Alpe Turati (Erba, Como) Famiglia Dactylioceratidae: Soc. Ital. Sci. Nat. Mem., v. 14, pt. 2, p. 85-136, 4 pls.

Pinna, Giovanni, and Levi-Setti, Franco, 1971, I Dactylioceratidae della Provincia Mediterranea (Cephalopoda Ammonoidea): Soc. Italiana Sci. Nat. e Mus. Civico Storia Nat. Milano Mem., v. 19, pt. 2, p. 49-136, pls. 1-12, 21 text-figs.

Quenstedt, F. A., 1856-1858, Der Jura: Tübingen, p. 1-208, pls. 1-24 (1856); p. 209-842, pls. 25-100 (1858).

-1882-1885, Die Ammoniten des Schwabischen Jura., v. 1, Der Schwarze Jura (Lias): Tübingen, p. 1-48, pls. 1-6 (1882); p. 49-96, pls. 7-12 (1883); p. 97-240, pls. 13-30 (1884); p. 241-440, pls. 31-54 (1885).

Reynes, P., 1868, Essai de géologie et de paléontologie aveyronnaises: Paris (Baillère), 110 p., 7 pls.

1879, Monographie des ammonites Lias: Marseilles and Paris, atlas, $58 \mathrm{pls}$. [text never published].
Sapunov, I. G., 1963, [Toarcian ammonites of the family Dactylioceratidae from western Bulgaria]: Bulgar. Akad. Nauk, Geol. Inst., Trudove Geol. Bulgar., Ser. Paleontologiya, v. 5, p. 109-136, 6 pls. (In Bulgarian; Russian and English summaries.)

Schlegelmilch, Rudolf, 1976, Die Ammonitendes sueddeutschen Lias: Stuttgart, Gustav Fischer Verlag, 212 p., 52 pls., 21 figs.

Schmidt-Effing, Reinhard, 1972, Die Dactylioceratidae, eine Ammoniten-familie des unteren Jura: Münstersche Forsch. Geologie und Paläontologie, No. 25 and 26, 216 p., 19 pls., 31 figs.

Sowerby, James, and Sowerby, J. de. C., 1812-46, Mineral conchology of Great Britain: London, B. Meredith, 7 v., pls. 1-383 (1812-22) by J. Sowerby; pls. 384-648 (1823-46) by J. de C. Sowerby.

Spath, L. F., 1925, Notes on Yorkshire ammonites: Naturalist, p. 107-112, 137-141, 167-172, 201-206, 263-269, 299-306, 327-331, 359-364.

Trueman, A. E., and Williams, D. M., 1925, Studies in the family Echioceratidae: Royal Soc. Edinburgh Trans., v. 53, p. 699-739, pls. 1-4.

Waehner, Franz, 1882-98, Beitrage zur Kenntnis der tieferen Zonen des unteren Lias in den nordöstlichen Alpen: Beitr. Paläont. Geol. Oster.-Ungarns v. des Orients, v. 2-11, 291 p., 66 pls.

Wright, Thomas, 1878-86, Monograph on the Lias ammonites of the British Islands: London, Palaeontographical Soc., p. 1-48, pls. 1-8 (1878); p. 49-164, pls. 9-18 (1879); p. 165-264, pls. 19-40 (1880); p. 265-328 (pls. 22A, B, 41-48 (1881); p. 329-400, pls. 49-52, 52A, 53-69 (1882); p. 401-440, pl. 70-77 (1883); p. 441-480, pls. 78-87 (1884); p. 481-503, pl. 88 (1886). 



\section{INDEX}

[Italic page numbers indicate descriptions and major references]

actaeon, Tropidoceras ___________ 18, 20,35, s7; pl. 8

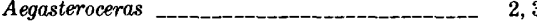
simile Aegoceras extracostatum portlocki

tenerum

Aichilik River

Alaska Peninsula

Alaska Range

$-10,12,31,33,34$

Alinchak Bay __11, 12, 28, 29, 30

Alsatites liasicus zone

Amaltheidae

Amaltheus margaritatus ______________ $17,19,37 ; \mathrm{pl} .10$ zone -

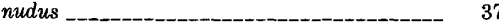

stokesi____ $7,17,18,19,21,37 ; \mathrm{pl} .10$ $\mathrm{sp}$ (Pseudoamaltheus) engelhardti - 17,38

Ammonite zones:

Hettangian

Pliensbachian -_-_-_-_- 11

Sinemurian

Toarcian

Ammonites pseudoradians

variabilis

Anatuvuk River

angulata, Schlotheimia

aplanatum, Paltechioceras

Apoderoceras

$18,21,35 ; \mathrm{pl}, 8$

Arctic Coastal Plain _._______ 12, 18

Arctoasteroceras _- $2,3,16,33$ jeletzkyi $3,16,22,31,33 ; \mathrm{pl} 5$ Argutarpites argutus argutum, Protogrammoceras _-____-_18, 21, 41; pl. 11 argutus, Argutarpites

argutus, Paltarpites

Arieticeras $\quad 2,18,39$ algovianum _..._. $20,39,40 ; \mathrm{pl} .10$ domarense ________________ $18,20,21,39 ; \mathrm{pl} .10$

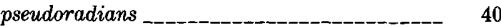
retrorsicosta sp Arieticeratinae _-_____- 39

Arietites bucklandi _._._. $12,13,17,32 ; \mathrm{pl} .4$ zone Arietitid genus undetermined _._____ 20,22 Arietitidae Arnioceras _._. $2,13,15,33$ densicosta _______ $15,20,22,38 ; \mathrm{pl} .5$ semicostatum ________________ 12,15 $\mathrm{sp}$

Asteroceras obtusum obusum Zone _-_-__-_ 12, 15 attenuatus, Dactylioceras _-_-________-_ 39

(Aveyroniceras) italicum, Prodactylioceras _-__._ 38

Badouxia

2,15 canadensis

22,$91 ;$ pl. 2

columbiae

Bajocian _... $7,10,11$

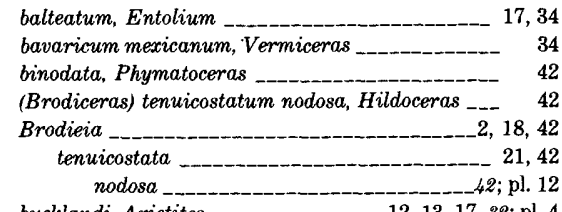

bucklandi, Arietites $12,13,17, s 2 ; \mathrm{pl} .4$

\section{C}

Caenisites

calcimontanum, Discamphiceras __________ 29, 30

(Caloceras), Psiloceras _-______________-_ 29

canadense, Psiloceras ___

canadensis, Badouxia __ 22,$31 ; \mathrm{pl}, 2$

carlottense, Fanninoceras

Catacoeloceras 2,39

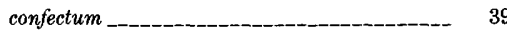

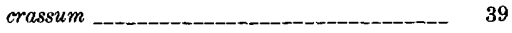
$\mathrm{sp}$ charlesi, Coroniceras (Paracoroniceras)

Charmasseiceras __ $2,13,15$ charmassei _-_-_-_ 32 marmoreum _- 17, 22, 31; pl. 3 posttaurinum 32 $\mathrm{sp}$ charmassei, Charmasseiceras _-_____________- 32

Chitina Valley

Chulitna River

Chulitna River area _-__-__-_-_-_ 10, 16

Chulitna Valley _.____________ 2, 33

Coeloceras italicum 38

lorioli

mucronatum

Coeloceratidae

Coleen quadrangle

colubriforme, Prodactylioceras _-____ 38

columbiae, Badouxia __

columbiae, Psiloceras (Cumviceras) __________ 31

commune, Dactylioceras _ 21, $\$ 8 ; \mathrm{pl}, 11$

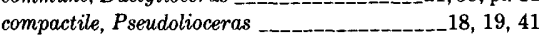

compressa, Haugia ________________21, 22, 4\%; pl. 12

confectum, Catacoeloceras

coregonense, Paracaloceras

Coroniceras haveri

multicostatum _... 32

$s p$

sp. $A$ _

sp. $B$

sp. C

(Paracoroniceras) 2, 2

charlesi ___ $\quad 32$

$\mathrm{sp}$

crassibundum, Dactylioceras 38

crassiusculosum, Dactylioceras __._________- 38

crassum, Catacoeloceras _._. 39

crucilobatum, Crucilobiceras ________ 17, 20,34; $\mathrm{pl} .5$

Crucilobiceras $2,15,17,34,36$ crucilobatum ___ $17,20,34 ; \mathrm{pl} .5$ densinodulum _______ 21,34 pl. 7

densinodum ___ 35

muticum 21, 35; pl. 7

pacificum _._ $18,20,35 ; \mathrm{pl} .8$

submuticum ____________________ 21,$35 ;$ pl. 7

sp

(Curviceras) columbiae, Psiloceras _._-___-_-_ 31 curviornatum, Waehneroceras

30

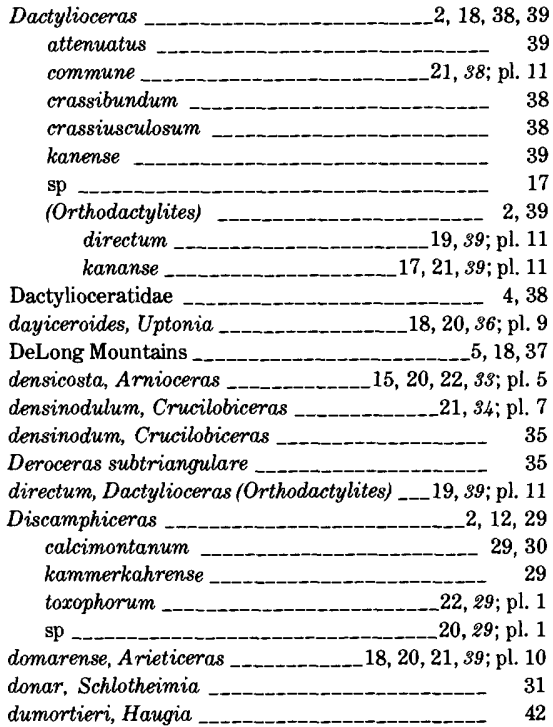

$\mathrm{E}$

Echioceras

raricostatum

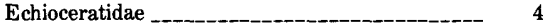

Eleganticeras __________ 41 $\mathrm{sp}$ Elegantuliceras _- 2 elegantulum ___________________ 41

elegantulum, Elegantuliceras _-__- 41

elicitum, Paltechioceras (Paltechioceras) -_-_-_ 34

engelhardti, Amaltheus (Pseudoamaltheus) -.._-_ 17, 38

Entolium balteatum _-_________________ 34

semiplicatum ________ 16, 17, 34

Eoderoceratidae _-_ 2,34

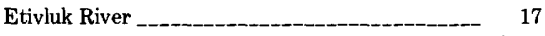

exaratum, Harpoceras _._________18, 19, 21, 40; pl. 11

extracostatum, Aegoceras _- 30

F

fallaciosum, Grammoceras ______________-_ 42

Fanninoceras 2,36 carlottense - 21,36 kunae _. 21,$36 ; \mathrm{pl} .7$ fontanellense, Fontanelliceras _......... 18, 21, 40; pl. 11

Fontanelliceras $\quad 2,18,40$ fontanellense -_____ 18, 21, 40; pl. 11 (Franziceras), Psiloceras __..____ 2, 7, 12, 29, 31 ruidum, Psiloceras _.._________12, 19, 22, 29; pl. 1 sp., Psiloceras $17,19,29 ; \mathrm{pl} 1$ fucini, Prodactylioceras italicum ___ 20,$38 ; \mathrm{pl} .10$ G

Glenn Shale $6,29,37$

grammicus, Macrogrammites _._- 30 


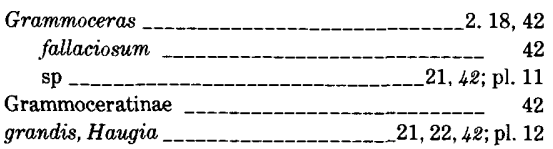

$\mathrm{H}$

Hammatoceratidae

$-2,42$ harbledownense, Paltechioceras _-_15, 17, 20, 21, 34; pl. 4 harbledownensis, Melanhippites 34 Harpoceras _-_-_-_-_ 18,40 exaratum _.__________-_18, 19, 21, 40; pl. 11 variabile

Harpoceratinae 40 haueri, Coroniceras _._- 32 Haugia $11,18,42$ compressa _ 21, 22, 48; pl, 12 dumortieri
grandis

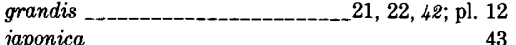
variabilis _ 21,$43 ; \mathrm{pl} .12$ $\mathrm{sp}$ Zone 18 Healy (A-6) quadrangle - $10,31,33,34$ Hettangian ___ $7,11,15,16,28$ ammonites $7,11,15,16,28$ Hildoceras (Brodiceras) tenuicostatum nodosa Hildoceratidae $\quad 2,4,39$

Howard Pass quadrangle I

ibex Zone, Tragophylloceras _____________ 18, 35, 37, 38 Ignek Creek

Ipnavik River italicum Coeloceras

Prodactylioceras _____ 18, 38

Prodactylioceras italicum _-_____-_20, $38 ; \mathrm{pl} .10$

Prodactylioceras (Aveyroniceras) _-_._-_-_-- 38

fucini, Prodactylioceras $\quad 20,38 ; \mathrm{pl}, 10$

italicum, Prodactylioceras __________ 20,$38 ; \mathrm{pl} .10$ Itkillik River

\section{$\mathrm{J}$}

jamesoni, Uptonia

$16,19,36 ; \mathrm{pl} .9$ japonica, Haugia jeletzkyi, Arctoasteroceras ___-____3, 16, 22, 31, 33; pl. 5

\section{K}

kammerkahrense, Discamphiceras $\quad 29$ kanense, Dactylioceras _- 39 Dactylioceras (Orthodactylites) _-___-_17, 21, 39; pl. 11

Kavik River Kenai

Kenai Peninsula -__ $10,12,29,32$ Kialagvik Formation _.__________ 11, 42, 43 Kingak Shale, ____ 5, 29, 31, 32, 33, 37, 39, 41 kunae, Fanninoceras _._._. 21,$36 ; \mathrm{pl}, 7$

L

Laqueoceras _-_._______ 12,30

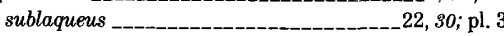
Leptaleoceras _ $2,18,40$

pseudoradians $18,21,40 ; \mathrm{pl} .11$ liasicus, Zone Alsatites _._-_-_...- 12 Lisburne Ridge lorioli, Coeloceras

Lubbe Creek Formation lythense, Pseudolioceras _-__-_-_ 18, 19,41 Lytoceras

M

maclintocki, Pseudolioceras

Macrogrammites grammicus margaritatus, Amaltheus marmoreum, Charmasseiceras

Matanuska River Valley

$17,22,31 ;$ pl. 3

McCarthy Formation

$34,35,36,37,38,40$

Melanhippites harbledownensis

mexicanum, Vermiceras bavaricum

Microderoceras

mitis, Orthodactylites

montana, Schlotheimia

mucronatum, Coeloceras

multicostatum, Coroniceras

Vermiceras

muticum, Cmucilobiceras

Nelchina

Nelchina area

Nodicoeloceras

nodosa, Brodieia tenuicostata

Hildoceras (Brodiceras) tenuicostatum

North Fork of the American River

nudus, A maltheus

obtusum, Asteroceras

Old Rampart

(Orthechioceras), Paltechioceras

radiatum, Paltechioceras

sp., Paltechioceras

Orthodactylites directum mitis

39

(Orthodactylites), Dactylioceras __-_-_-_-_-_-_-_ 2 directum, Dactylioceras _-_-_-__-_-_19, $39 ; \mathrm{pl} .11$ kanense, Dactylioceras

$17,21,39 ;$ pl. 11

Otapiria

tailleuri -_-_-__ $5,16,17$

Oxynoticeras oxynotum _-________-_ 3, 16

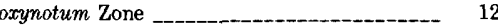

Oxynoticeratidae -

oxynotum, Oxynoticeras _._- 3,16

\section{$\mathbf{P}$}

pacificum, Crucilobiceras

Paltarpites

argutus _-_.._- 41

paitus

Paltechioceras

aplanatum

harbledownense

$s p$ -

radiatum

$s p$

(Paltechioceras) elicitum _tomoceras ____ 21, 41; pl. 12 paltus, Paltarpites Paracaloceras _-_ 2,15 coregonense rursicostatum

$13,22,33 ; \mathrm{pl} .6$

(Paracoroniceras) Coroniceras $\quad 2,15$

Parson Bay _-_-_-_ 17

Phymatoceras _-_- 2,42

binodata

tumefacta

$\mathrm{sp}$

planorbis, Psiloceras ____________________20, 22, 28; pl. 1

Platypleuroceras sp

Pleuroceras spinatum Zone _-_-_-_-_ 38

plicatum, Psiloceras (Psiloceras) _._._-_-_-. 29

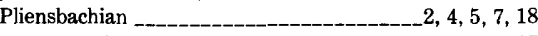

ammonites 17

Point Barrow area 12,37

Polymorphitidae

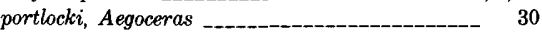

Schlotheimia (Waehneroceras) ___ 30

Waehneroceras _-_____ 30 ; pl. 2

posttaurium, Charmasseiceras _-_ 32
Prodactylioceras _._-_-_-_-_-_, 2, 38

colubriforme -_-_-_-_-_-_-_-_-_-_._-_.- 38

italicum 18, 38

fucini

italicum ___

sp -_-_-_-_-_-_-_ 20

(Aveyroniceras) italicum _-_-_- 38

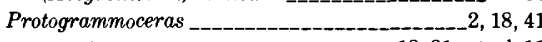
argutum _______ $18,21,41 ; \mathrm{pl}, 11$

paltum $18,21,41 ; \mathrm{pl} .12$

(Pseudoamaltheus) engelhardti, Amaltheus _._._- 17, 38

Pseudolioceras _compactile _-__-_-_ $18,19,41$

lythense $\quad 18,19,41$

maclintocki _-_-_-_-_-_-_-_-_-_-_-_-_ 41

whiteavesi _-________________- 11

sp _-_____ 21, 22 41; pl. 11, 12

pseudoradians, Ammonites _-__________._..... 40 Arieticeras -_-_-_-_-_-_-_-_-_-_-_ 40 Leptaleoceras ____________________ 18, 21, 40; pl. 11

Psiloceras canadense planorbis __________ 22, 28; pl. 1

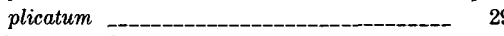

Psiloceras (Psiloceras) _.____________ 6, 12, 28, 29, 31

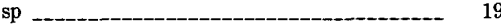

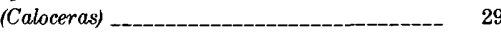

$\begin{array}{lr}\text { (Curviceras) columbiae -- } & 31 \\ \text { (Franziceras) } & \end{array}$

ruidum $12,22,29 ; \mathrm{pl} .1$

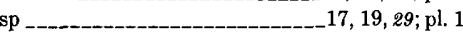

(Psiloceras), Psiloceras _-_-_-_-_-_6, 12, 28, 29, 31

Psiloceratidae

Puale Bay _. $2,11,12,13,18,28$

Puale Bay area

$29,30,31,33,42,43$

Q

Queen Charlotte Islands

$2,35,36,37$

$\mathrm{R}$

radiatum, Paltechioceras (Orthechioceras) _..._... 34 Radstockiceras _-Rainy Pass _._-_-_-_ 10 raricostatum zone, Echioceras _________ 4, 13, 15, 34, 35 Red Hill

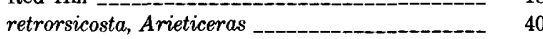
ruidum, Psiloceras (Franziceras) ___12, 19, 22, 29; pl. 2 rursicostatum, Paracaloceras _ 13, 22, 33; pl. 6

Sadlerochit River

Sagavanirktok River

Schlotheima $2,12,31$

angulata Zone _-_- 12

domar

montana

sp

(Waehneroceras) portlocki

Schlotheimiidae

Seldovia

Seldovia area _-_._. $12,15,29,32,33$

Seldovia Bay _-_-_-_-_ 10

semicostatum, Armioceras

semiplicatum, Entolium _-_._-_ 16, 17, 34

Shellabarger and Rainy Pass areas _-______- 10

Shellabarger Pass

simile, Aegasteroceras

Simpson test well No. 1

Sinemurian

ammonites

South Barrrow test well No. 12

South Barrow test well No. 3

Spike Mountain

spinatum, Pleuroceras 38

stokesi, Amaltheus _-_-_ 7, 17, 18, 19, 21, s7; pl. 10

sublaqueus, Laqueoceras _-_-_-_-_-_-__-_22, 30; pl. 3

submuticum, Crucilobiceras ______________21, 35; pl. 7 
subtriangulare, Apoderoceras

$18,21,35 ; \mathrm{pl} .8$

Deroceras

\section{$\mathrm{T}$}

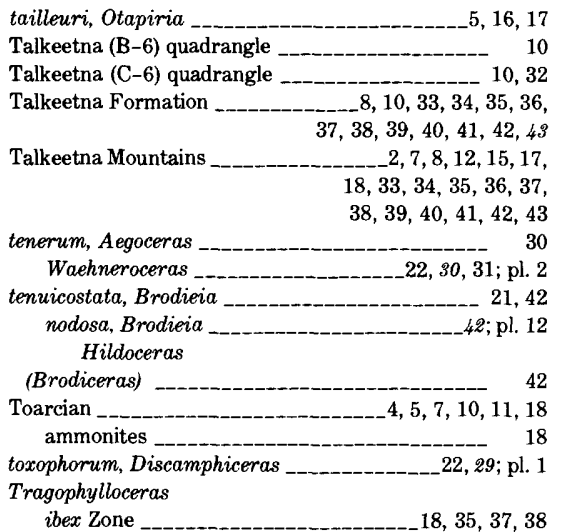

Triassic

Tropidoceras actaeon tumefacta, Phymatoceras

turneri, Caenisites

\section{$\mathrm{U}$}

Uptonia

dayiceroides
jamesoni
Zone
sp
sp. A
sp. B

$$
\text { sp. B }
$$

$\mathrm{V}$

variabile, Harpoceras variabilis, Ammonites

$$
\text { Haugia }
$$

Vermiceras bavaricum mexicanum multicostatum

\section{W}

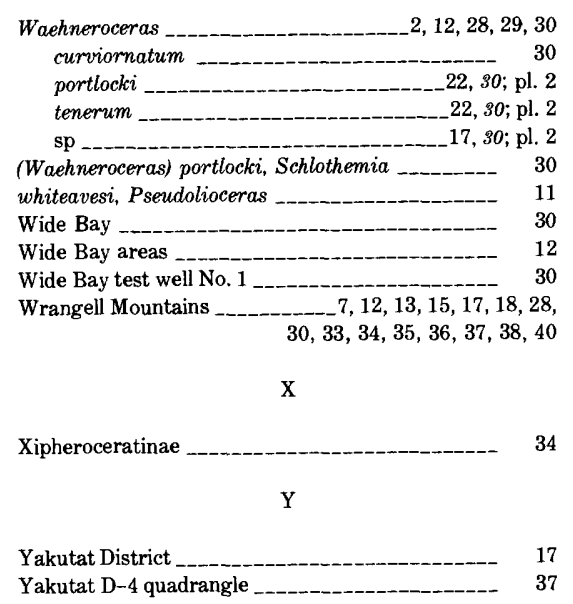





\section{PLATES 1-12}

Contact photographs of the plates in this report are available, at cost, from U.S. Geological Survey Library, Federal Center,

Denver, Colorado 80225 


\section{PLATE 1}

[Figures natural size unless otherwise indicated]

Figures 1, 2. Psiloceras cf. P. planorbis (J. de C. Sowerby) (p. 28).

1. Specimen, USNM 247950, from USGS Mesozoic loc. 29891.

2. Specimen, USNM 247951, from USGS Mesozoic loc. M1738.

3, 4, 8-10. Discamphiceras cf. D. toxophorum Waehner (p. 29).

3. Specimen, USNM 247960, from Richfield Oil Co. loc. 3002.

4. Specimen, USNM 247958, from USGS Mesozoic loc. 3110.

8. Specimen, USNM 247961, from Richfield Oil Co. loc. 3002.

9, 10. Crushed fragment and rubber imprint of external mold of one specimen, USNM 247959, from USGS Mesozoic loc. 25694. 5-7. Discamphiceras sp. (p. 29).

Rubber imprints of three external molds, USNM 247962, from USGS Mesozoic loc. 29890.

11, 15-17. Psiloceras (Franziceras) sp. ind. (p. 29).

$11,15,16$. Ventral and lateral views of specimen, USNM 247956, from South Barrow test well No. 12 at depth of $2,170.5 \mathrm{ft}$ (661.4 m).

17. Specimen, USNM 247957 from USGS Mesozoic loc. 29742.

12-14,18-24. Psiloceras (Franziceras) cf. $P$. (F.) ruidum Buckman (p. 29).

12. Rubber imprint of external mold $(\times 2)$ of a specimen, USNM 247952, from USGS Mesozoic loc. 29738.

13, 14. Rubber imprint of external mold ( $\times 2$ ), specimen, USNM 247954, from USGS Mesozoic loc. 29738.

18-20. Rubber imprint of external mold and views of internal mold of a specimen, USNM 247955, from USGS Mesozoic loc. 21242.

21-24. Rubber imprints $(\times 2)$ and suture line $(\times 4)$ of one specimen, USNM 247953, from USGS Mesozoic loc. 29738. 
GEOLOGICAL SURVEY

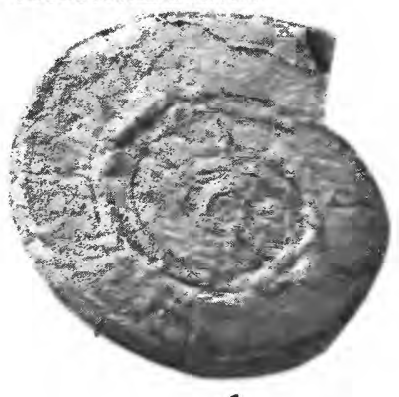

1

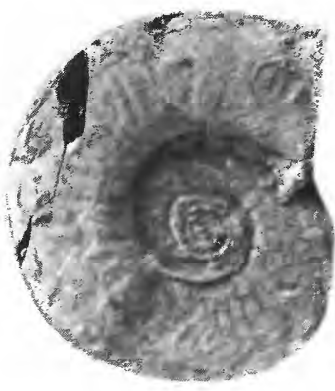

2

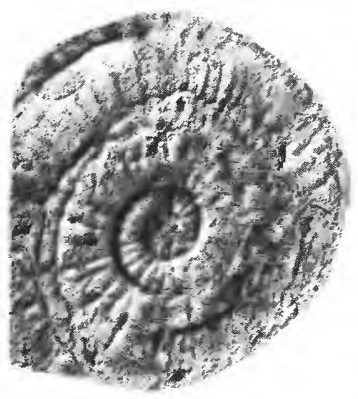

5

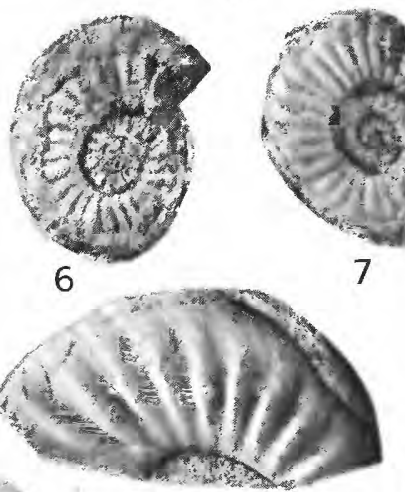

8
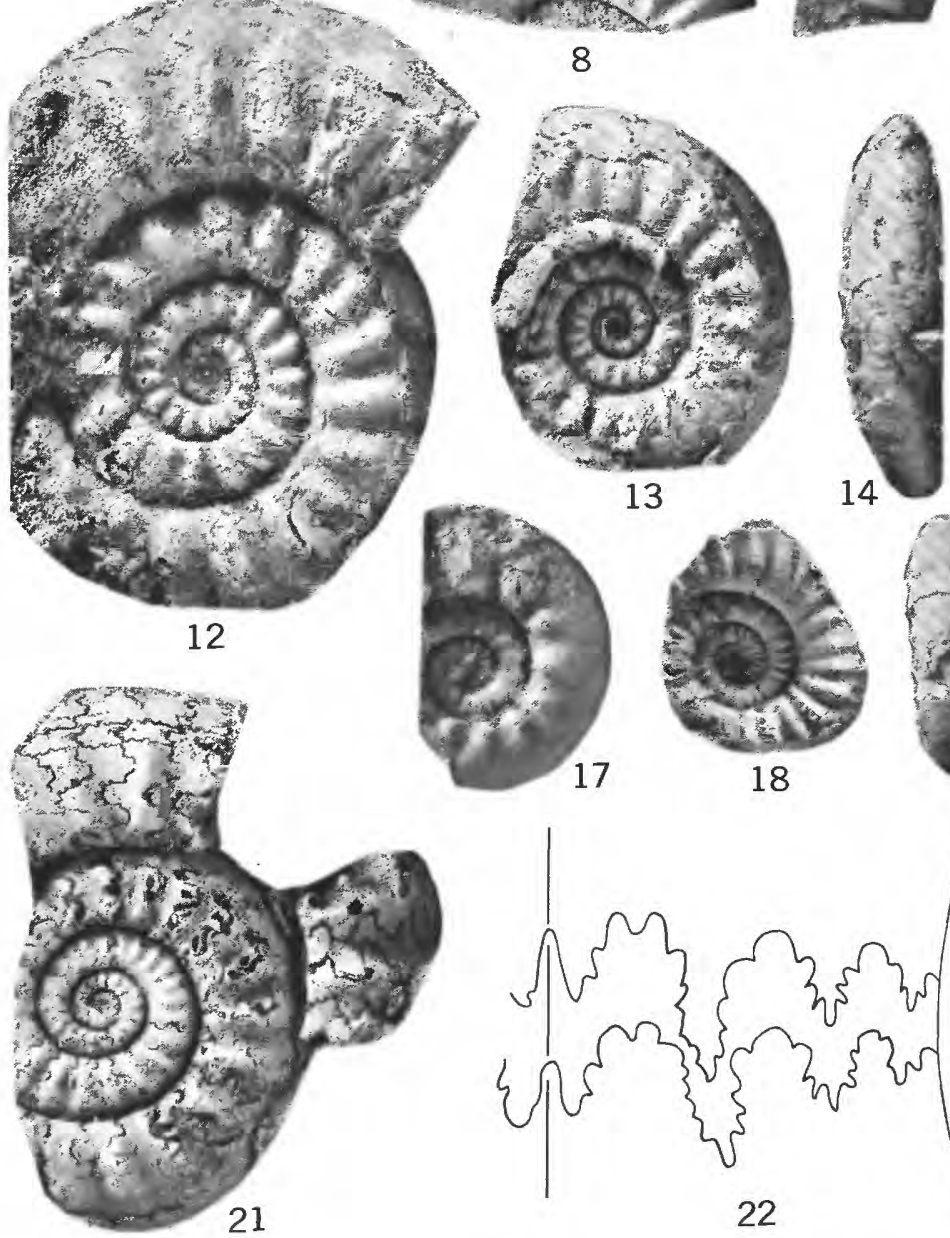

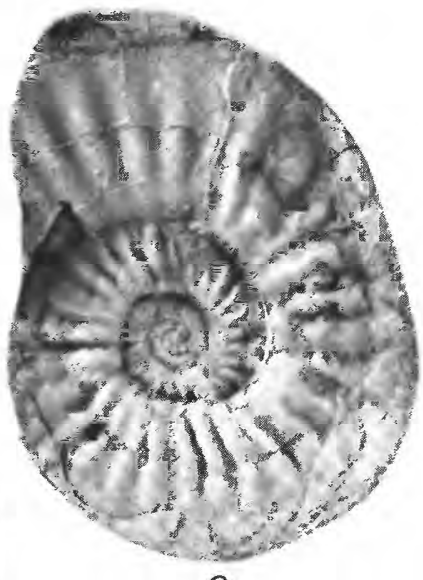

3
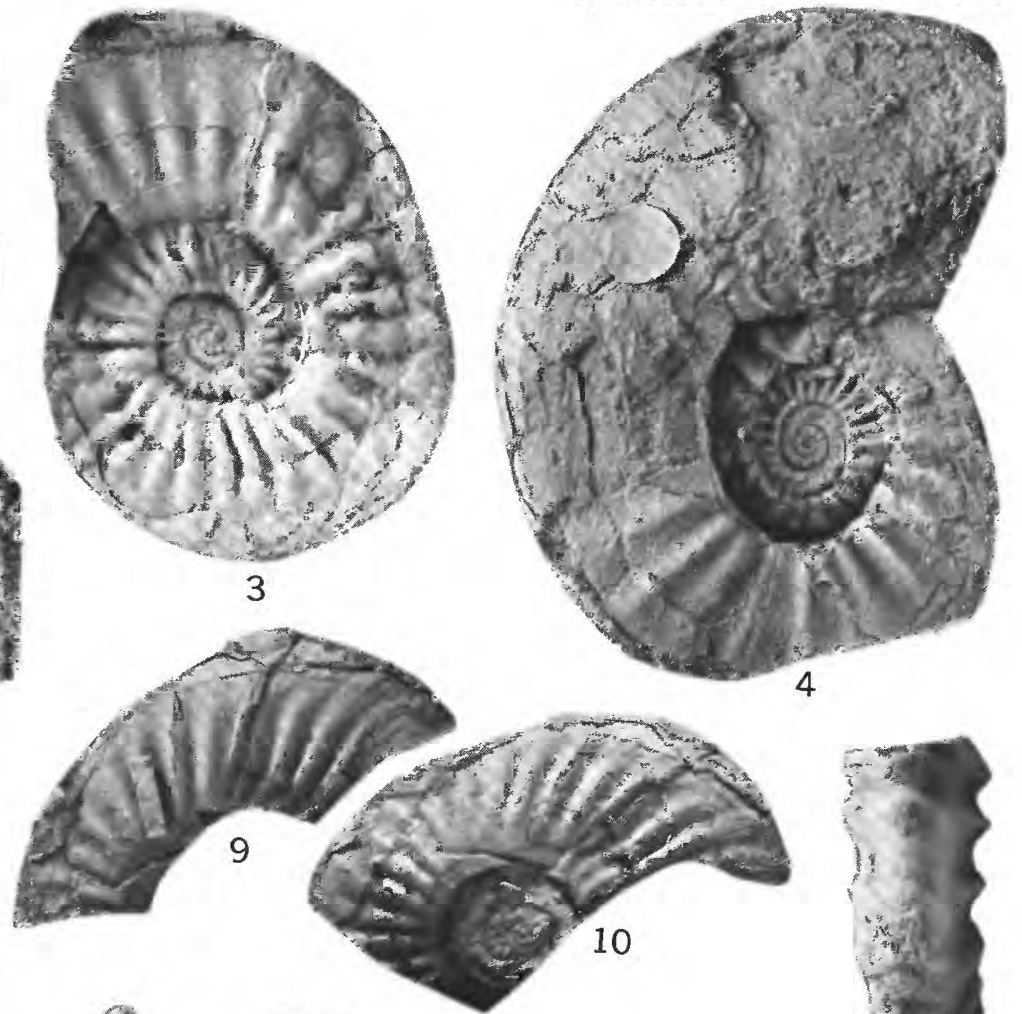
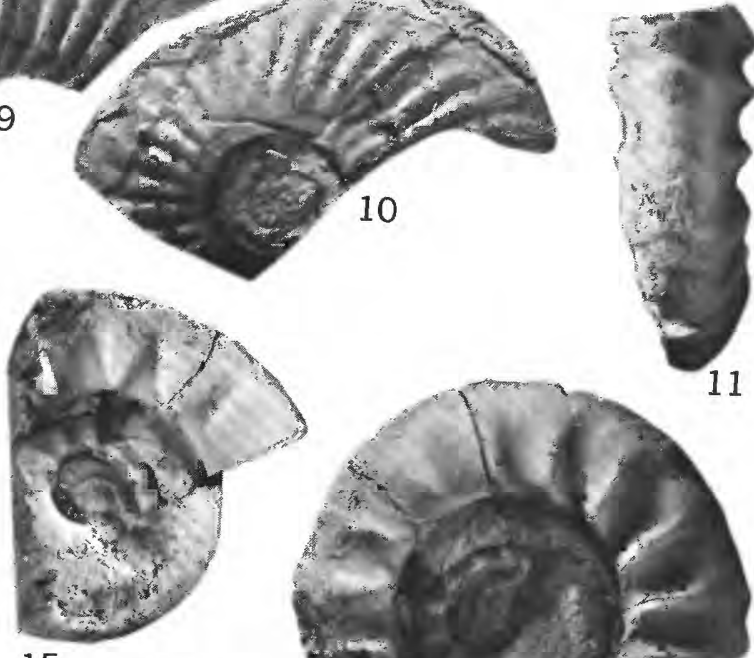

15
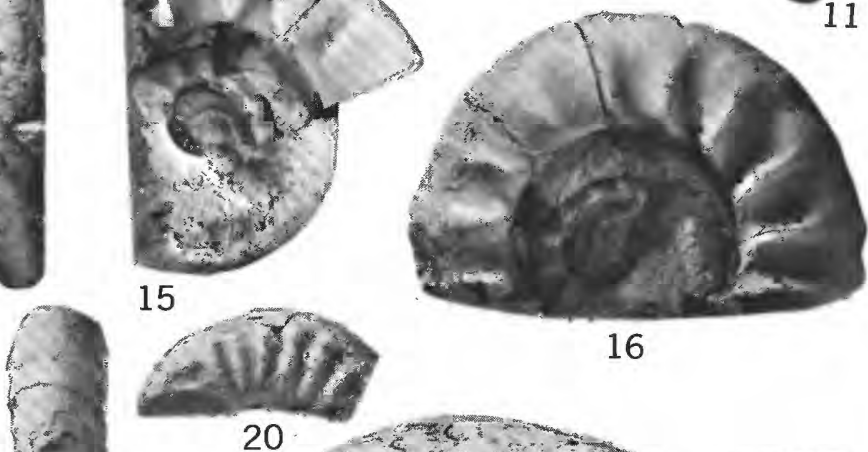

16
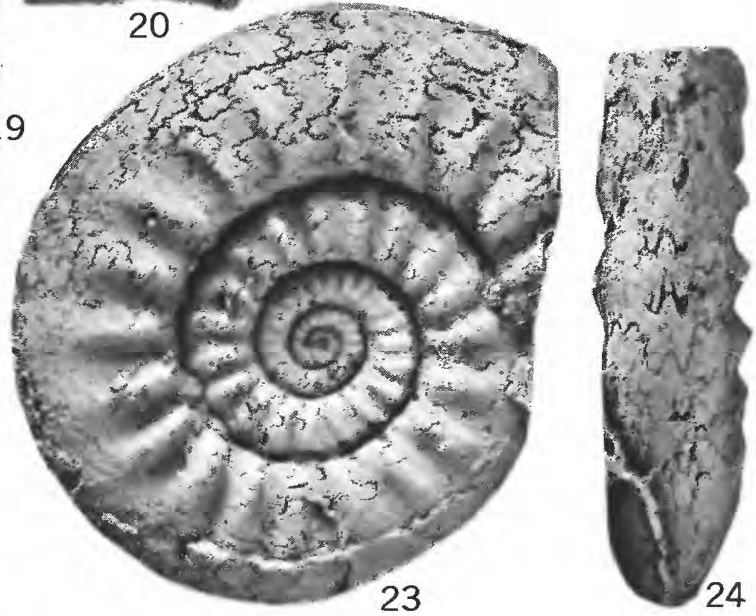

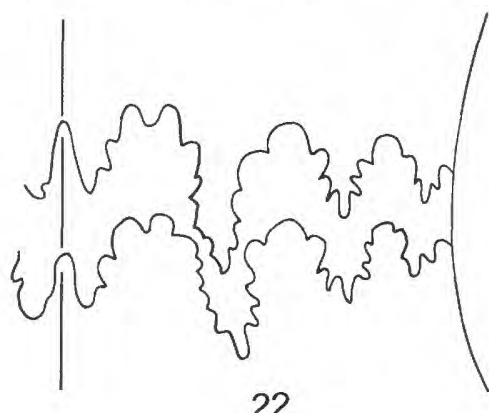

22

PSILOCERAS, P. (FRANZICERAS), AND DISCAMPHICERAS 


\section{PLATE 2}

[Figures natural size unless otherwise indicated]

Figures 1-6. Waehneroceras cf. W. tenerum (Neumayr) (p. 30).

1-3. Three specimens, USNM 247964, from Richfield Oil Co. loc. 3002. Figure 1 is a rubber imprint of an external mold.

4, 5. Specimen, USNM 247965, from USGS Mesozoic loc. 19803. Figure 5 below fracture is an external mold.

6. Specimen, USNM 247966, from USGS Mesozoic loc. 25694.

7, 10-15. Waehneroceras cf. W. portlocki (Wright) (p. 30).

7. Specimen, USNM 247971, from USGS Mesozoic loc. 25694.

10. Specimen, USNM 247969, from USGS Mesozoic loc. 12075.

11. Specimen, USNM 247970, from USGS Mesozoic loc. 12394.

12. Specimen, USNM 247967, from Richfield Oil Co. loc. 3002.

13. Specimen, USNM 247968, from USGS Mesozoic loc. 3110.

14, 15. Two specimens, USNM 247972, from Richfield Oil Co., Wide Bay test No. 1 at depth of 2,235-2,236 ft (681 m).

8, 9. Waehneroceras? sp. (p. 30).

Ventral and lateral views $(\times 2)$ of specimen, USNM 247973, from the South Barrow test well No. 12 at depth of 2,181.5 ft $(665 \mathrm{~m})$. 16, 17. Schlotheimia sp. (p. 31).

Specimen, USNM 247974, from USGS Mesozoic loc. 10820.

18-21, 24-28. Badouxia canadensis (Frebold) (p. 31)

18, 19. Hypotype, USNM 247977, from Richfield Oil Co. loc. 1240.

20, 21. Hypotype, USNM 247976, from USGS Mesozoic loc. 31266.

24, 28. Lateral views of hypotype, USNM 247975, from USGS Mesozoic loc. 31372. Figure 24 is a rubber imprint of the external mold.

25-27. Specimen, USNM 247975, from USGS Mesozoic loc. 31266.

22, 23. Badouxia columbiae (Frebold) (p. 31).

Hypotype, USNM 247978, from USGS Mesozoic loc. 31264.

22, 23. Hypotype, USNM 247978, from USGS Mesozoic loc. 31264. 
GEOLOGICAL SURVE
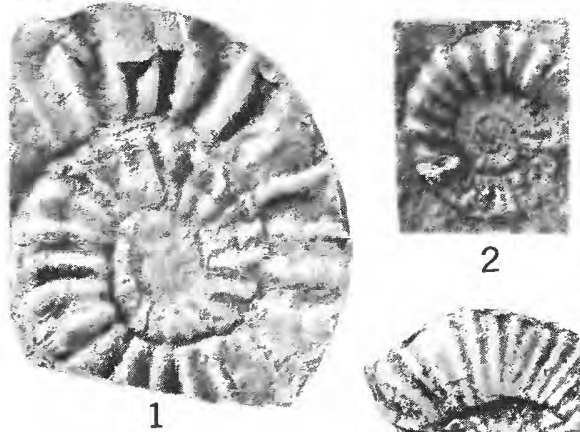

2
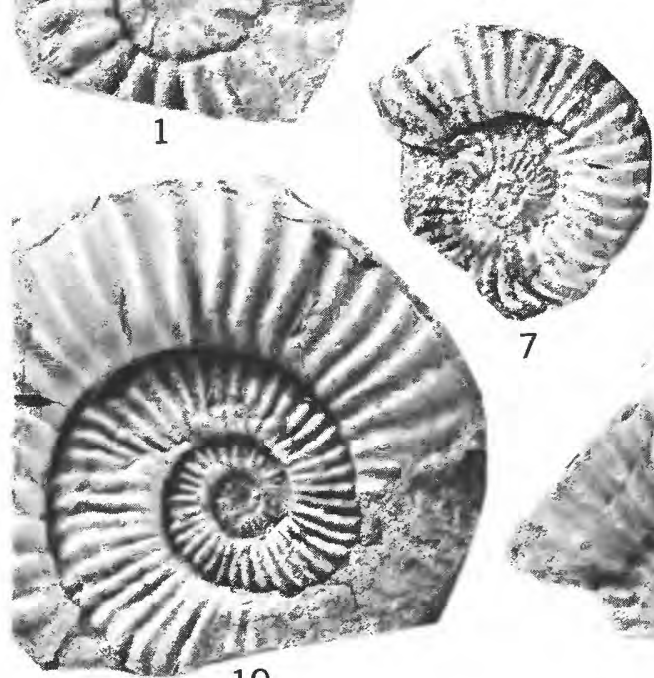

10

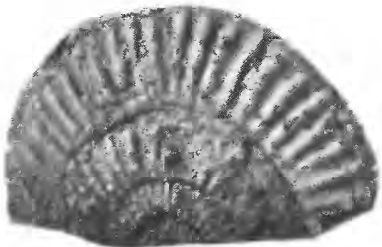

14

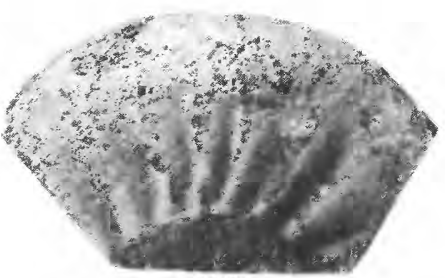

18
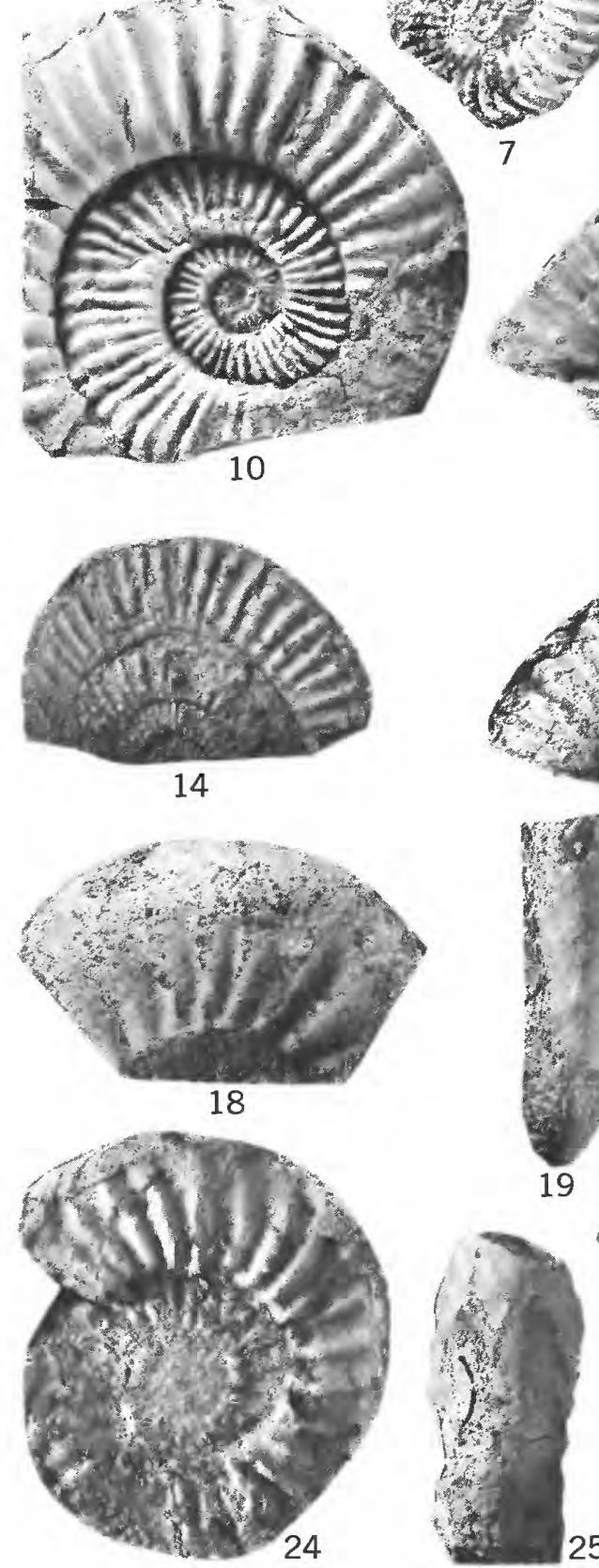

(1)
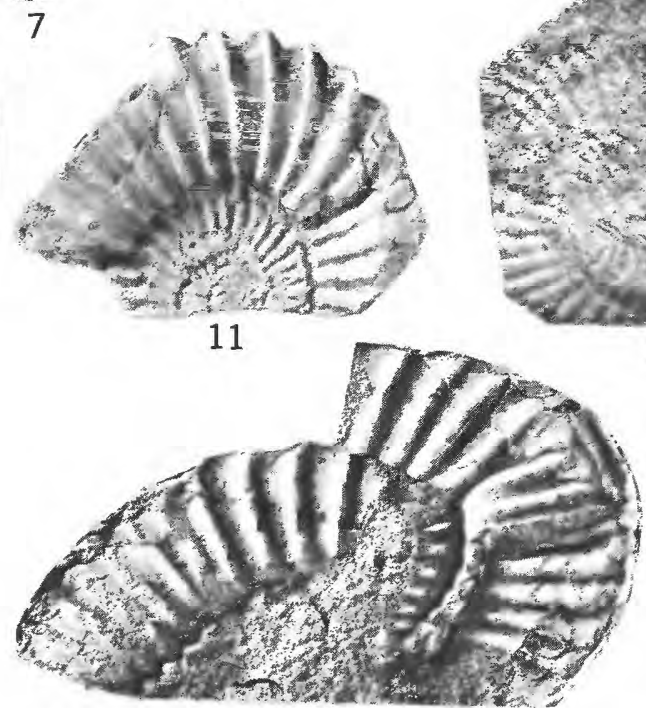

12

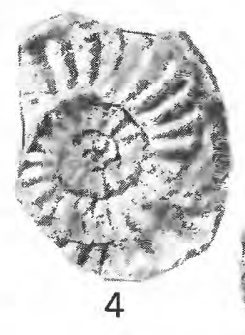

nown
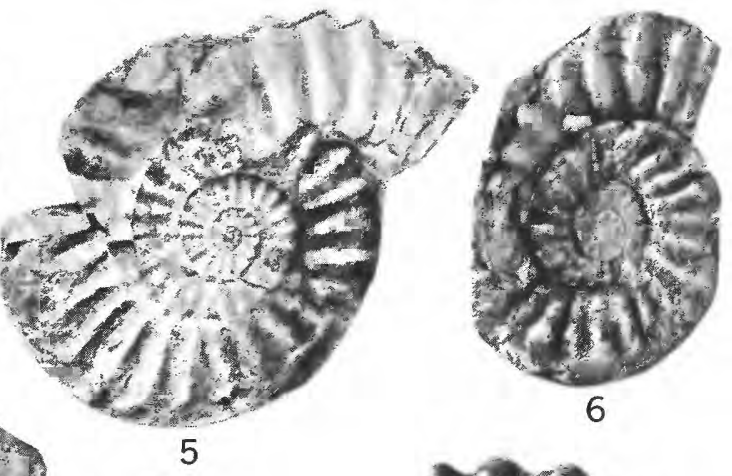

9
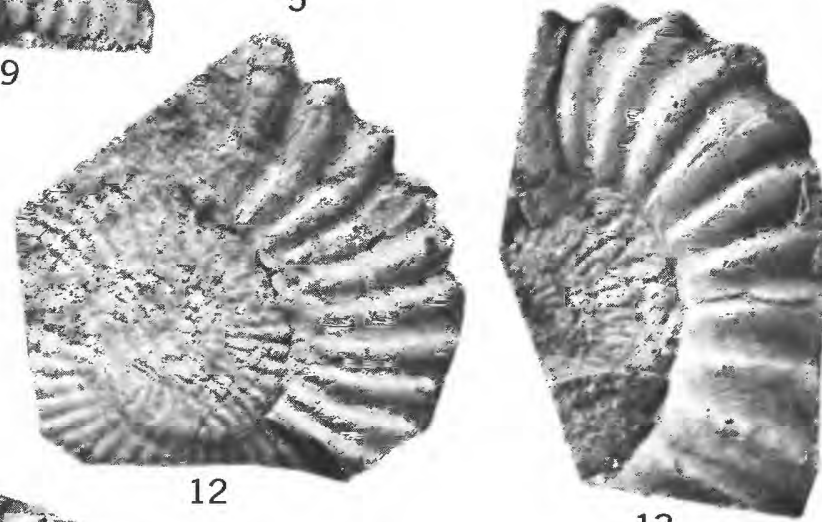

13
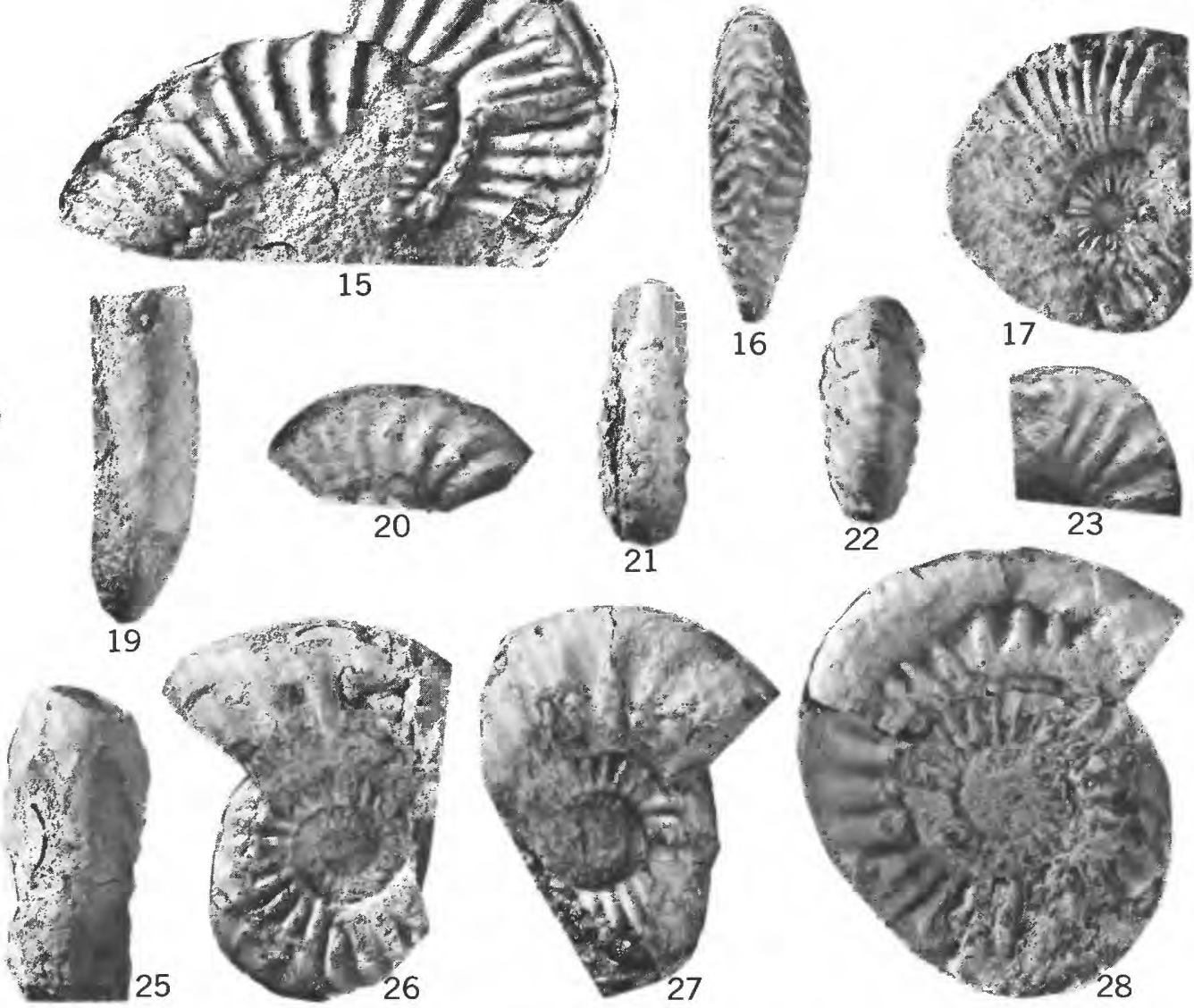

WAEHNEROCERAS, SCHLOTHEIMIA, AND BADOUXIA 


\section{PLATE 3}

[Figures natural size unless otherwise indicated]

FiguREs 1-3. Charmasseiceras cf. C. marmoreum (Oppel) (p. 31).

Lateral views of three specimens, USNM 247979, from the South Barrow test well No. 12 at depth of 2,061.5 ft (628 m).

4-10. Charmasseiceras sp. (p. 31).

$4-6,9,10$. Suture line $(\times 4)$ and lateral and ventral views of same specimen $(\times 2$ and $\times 6)$, USNM 247981 , from the South Barrow test well No. 3 at depth of $2,412 \mathrm{ft}(735 \mathrm{~m})$.

7, 8. Lateral and ventral views of specimen, USNM 247980, from the South Barrow test well No. 12 at depth of $2,056 \mathrm{ft}(627 \mathrm{~m})$. 11, 12. Paltechioceras (Orthechioceras?) sp. (p. 34).

Rubber imprint of external mold (fig. 11) and ventral view of specimen, USNM 248003, from USGS Mesozoic loc. 31261.

13. Laqueoceras cf. L. sublaqueus (Waehner) (p. 30).

Rubber imprint of external mold, USNM 247963, from Richfield Oil Co. loc. 3002. 

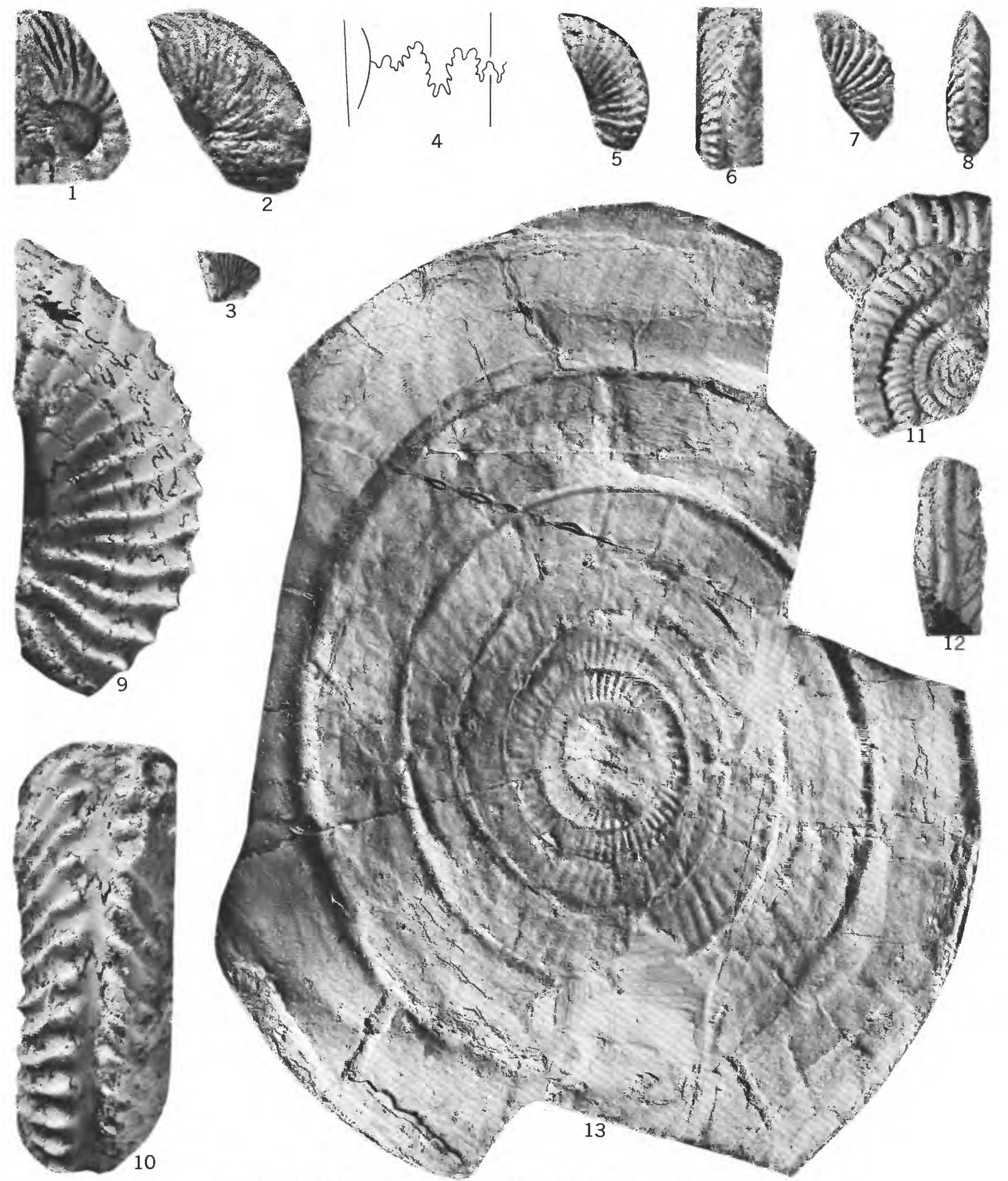

CHARMASSEICERAS, PALTECHIOCERAS, AND LAQUEOCERAS 


\section{PLATE 4}

[All figures are natural size]

Figures 1-5. Coroniceras sp. A (p. 32).

Three specimens, USNM 247982, from USGS Mesozoic loc. 30908. Ventral and lateral views of one specimen are shown in figures 1 and 3. Same views of another specimen are shown in figures 2 and 4.

6-10. Coroniceras sp. B (p. 32).

6. Rubber imprint of external mold of specimen, USNM 247983, from the South Barrow test well No. 12 at depth of 1,987.5 $\mathrm{ft}$ $(605.8 \mathrm{~m})$

7-10. Two specimens, USNM 247984, from the South Barrow test well No. 12 at depth of 1,987.6 feet (605.8 m). Ventral and lateral views of one specimen are shown in figures 7 and 10. Same views of another specimen are shown in figures 8 and 9. 11-13. Arietites cf. A. bucklandi (Sowerby) (p. 32).

Figure 13 probably represents an inner whorl of specimen shown in figures 11 and 12, USNM 108778, from the Avak test well No. 1 at depth of $1,836 \mathrm{ft}(560 \mathrm{~m})$.

14. Coroniceras sp. C (p. 32).

Rubber imprint of external mold, USNM 247985, from the South Barrow test well No. 3 at depth of $2,470 \mathrm{ft}(753 \mathrm{~m})$.

15-22. Paltechioceras cf. P. harbledownense (Crickmay) (p. 34).

15, 16. Rubber imprint and external mold of specimen, USNM 248000, from USGS Mesozoic loc. 31174.

17, 22. Specimens, USNM 248001, from USGS Mesozoic loc. 30139.

18-21. Ventral and lateral views of two specimens, USNM 248002, from USGS Mesozoic loc. 28661.

23-25. Coroniceras (Paracoroniceras) sp. (p. 32).

Two specimens, USNM 247986, from USGS Mesozoic loc. 31128. Figure 22 represents a rubber imprint of the inner whorls. Figures 23 and 24 show lateral and ventral views of an outer whorl. 
GEOLOGICAL SURVEY

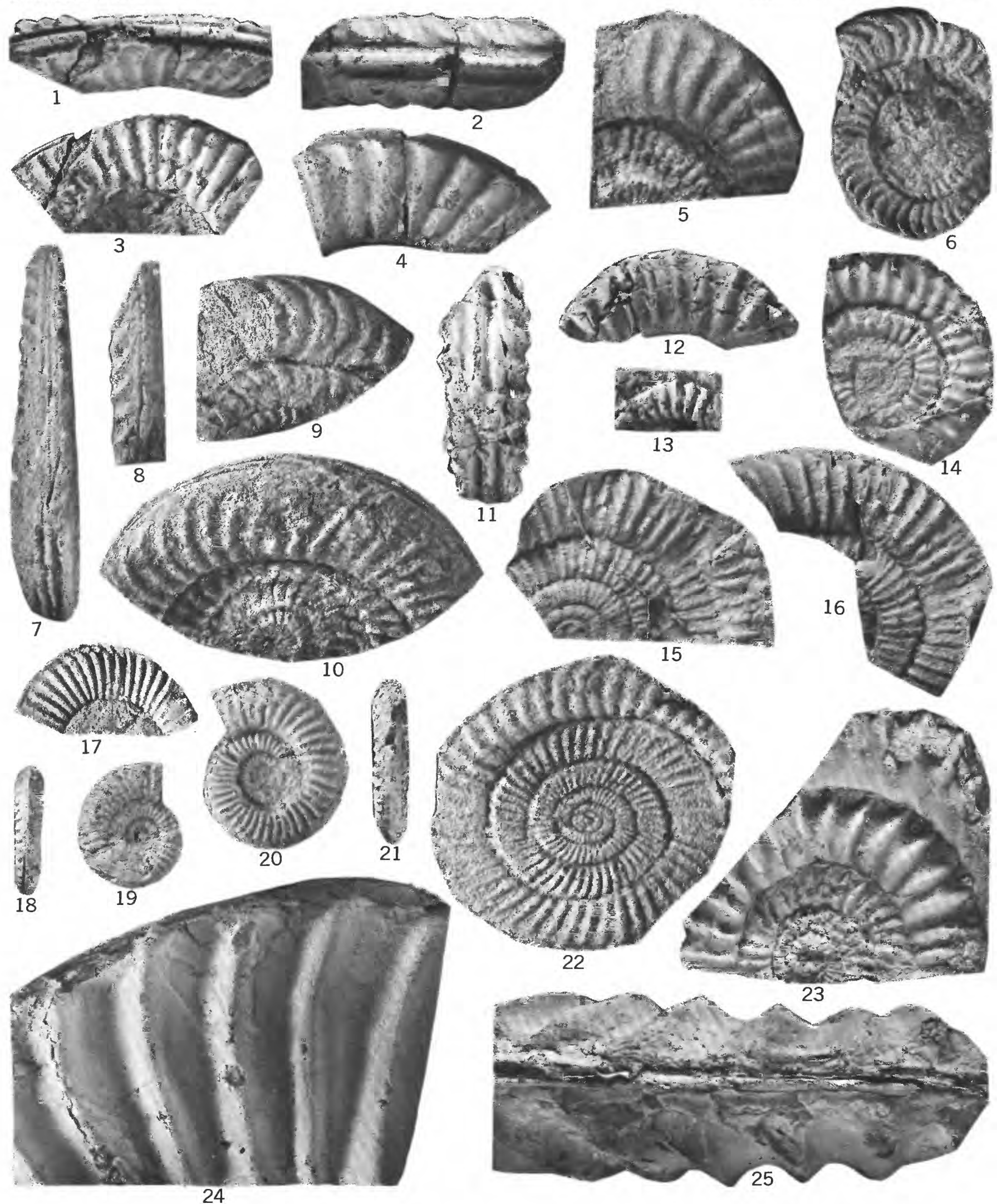

CORONICERAS, C. (PARACORONICERAS), ARIETITES, AND PALTECHIOCERAS 


\section{PLATE 5}

[Figures natural size unless otherwise indicated]

FiguRes 1-4. Arctoasteroceras jeletzkyi Frebold (p. 33).

1, 2. Hypotype, USNM 247999, from USGS Mesozoic loc. 31261.

3, 4. Hypotype, USNM 247998, from USGS Mesozoic loc. 31261.

5, 6, 12-15. Arnioceras sp. juv. (p. 33).

5,6 . Ventral and lateral views.

12,13 . Ventral and lateral views.

14,15 . Rubber imprints of two external molds showing smooth inner whorls. Specimens, USNM 247990, from the South Barrow test well No. 12 at depth of $2,056 \mathrm{ft}(626.7 \mathrm{~m})$.

7, 8. Crucilobiceras cf. C. crucilobatum Buckman (p. 34).

7. Laterally.crushed small specimen ( $\times$ 2), USNM 248005, from USGS Mesozoic loc. 14030.

8. Rubber imprint of external mold of large specimen, USNM 248004, from USGS Mesozoic loc. 14472.

9-11, 16-24. Arnioceras cf. A. densicosta (Quenstedt) (p. 33).

$9,16,17$. Rubber imprint of inner whorls (fig. 9) and suture line ( $\times 2$ ) of one specimen, USNM 247987, from USGS Mesozoic loc. 16229.

10, 11, 18, 19, 22-24. Five specimens, USNM 247988, from USGS Mesozoic loc. 16229. Ventral and lateral views of one specimen are shown in figures 10 and 11. Same views of another specimen are shown in figures 18 and 19. Figures 22-24 represent rubber imprints of external molds.

20, 21. Lateral and ventral view of specimen, USNM 247989, from USGS Mesozoic loc. 21237. 

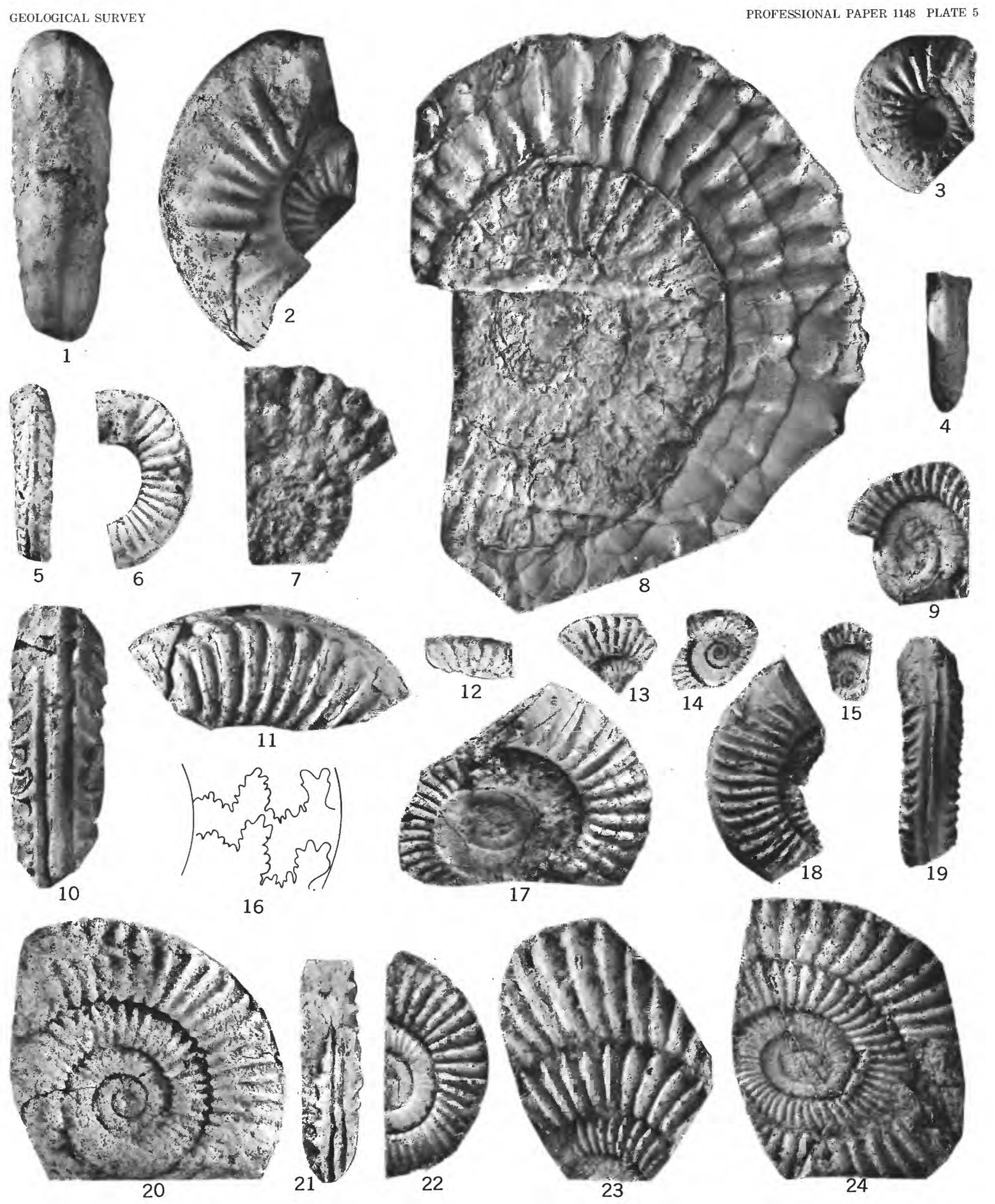

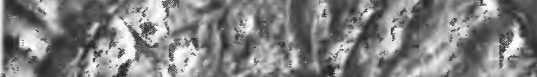

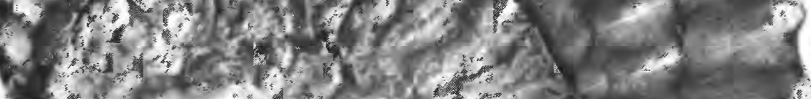


PLATE 6

[All figures are natural size]

Figures 1-11. Paracaloceras rursicostatum Frebold (p. 33).

1, 2. Hypotype, USNM 247991, from USGS Mesozoic loc. 12396.

3, 5. Hypotype, USNM 248070, from USGS Mesozoic loc. 31372.

4. Hypotype, USNM 247996, from USGS Mesozoic loc, 10820.

6. Hypotype, USNM 247997, from USGS Mesozoic loc. 10820.

7, 11. Hypotype, USNM 247993, from Richfield Oil Co. loc. 1241.

8. Hypotype, USNM 247995, from USGS Mesozoic loc. 31266.

9. Hypotype, USNM 247994, from USGS Mesozoic loc. 2981.

10. Hypotype, USNM 247992, from USGS Mesozoic loc. 12396. 
GEOLOGICAL SURVEY
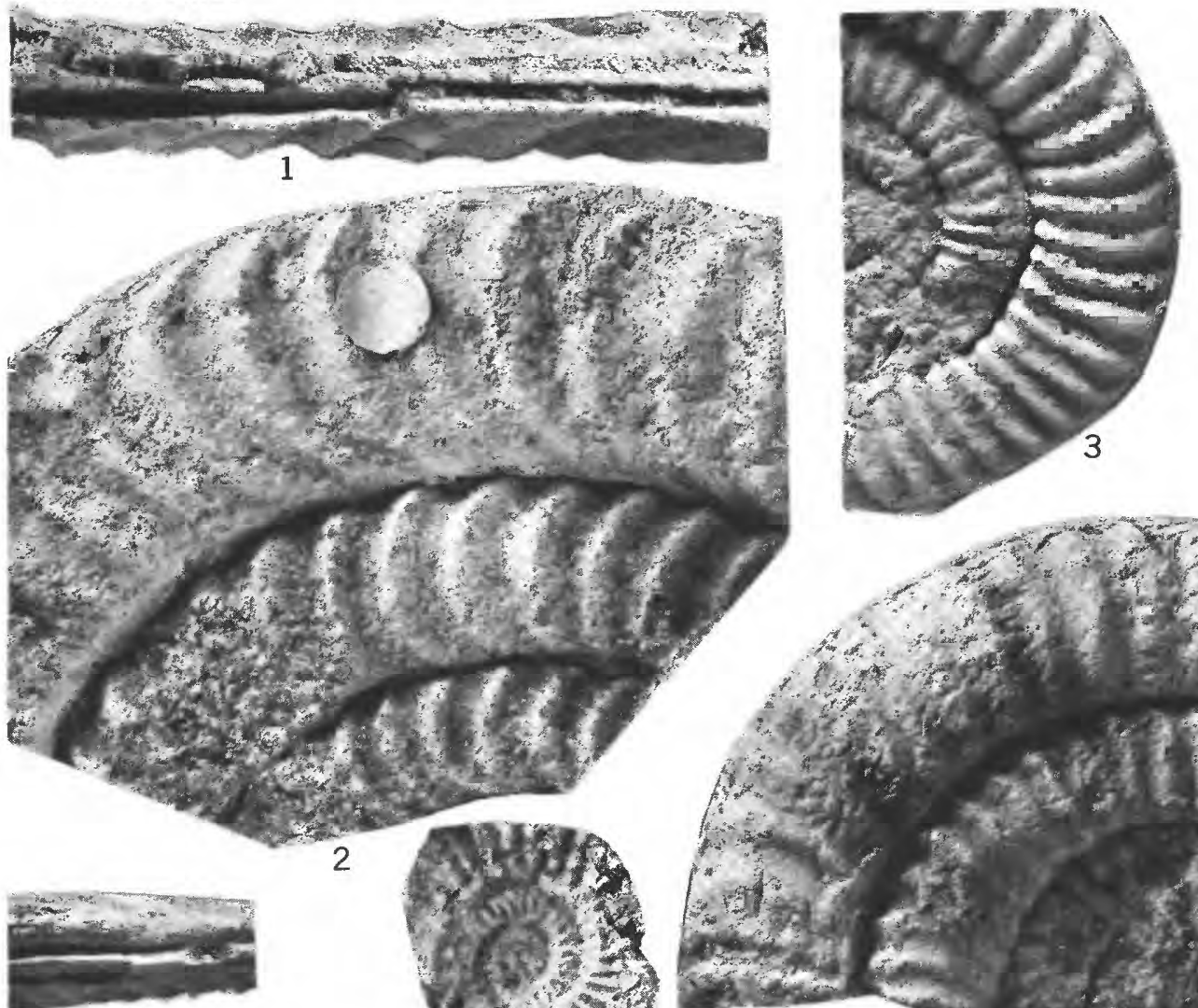

5

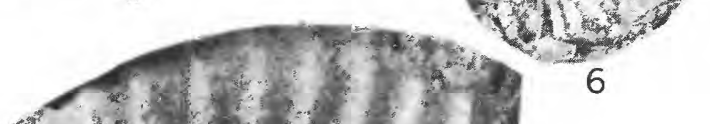

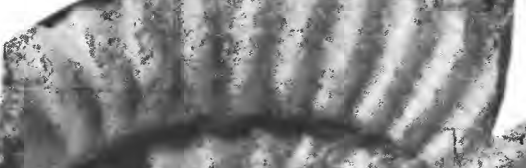
2
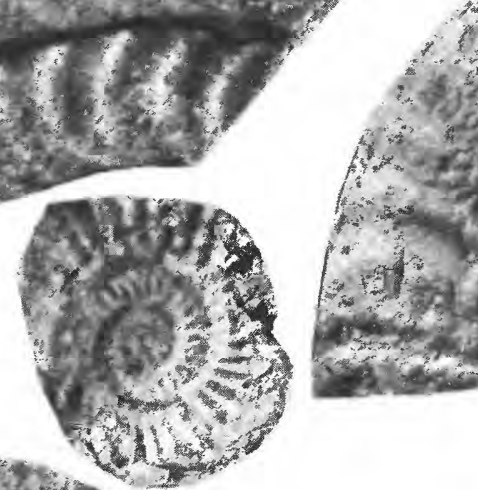

1. 6

PROFESSIONAL PAPER 1148 PLATE 6
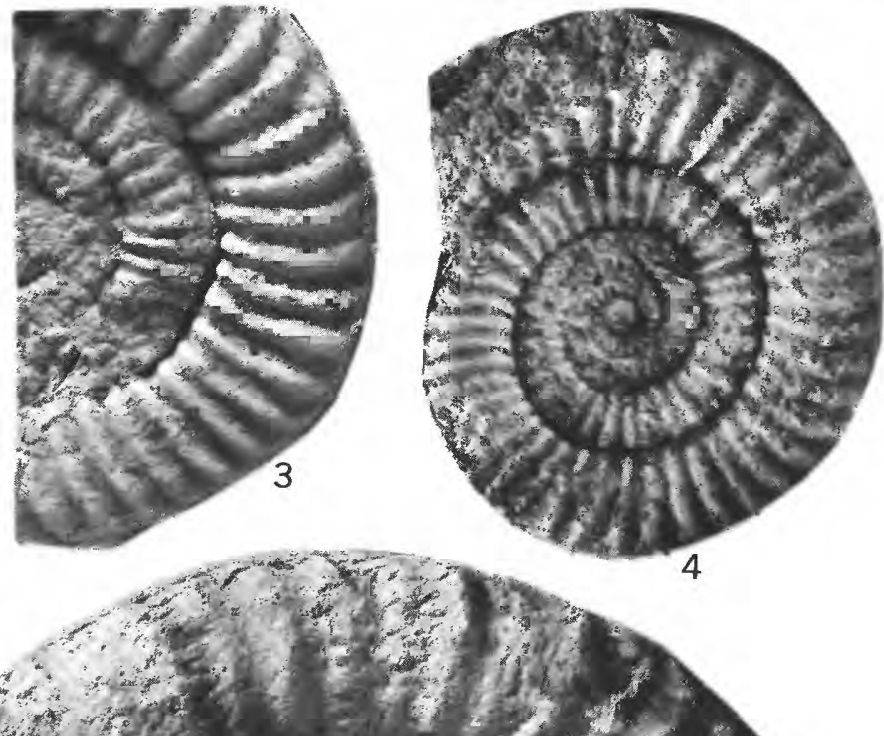

$x+y$

trast

23

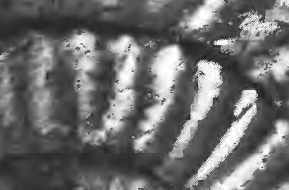

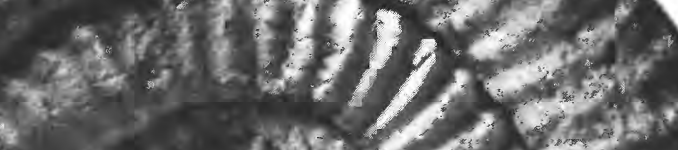

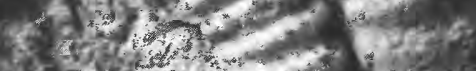

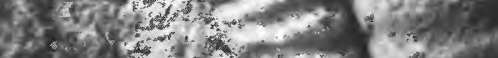

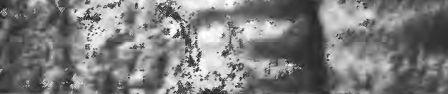
7
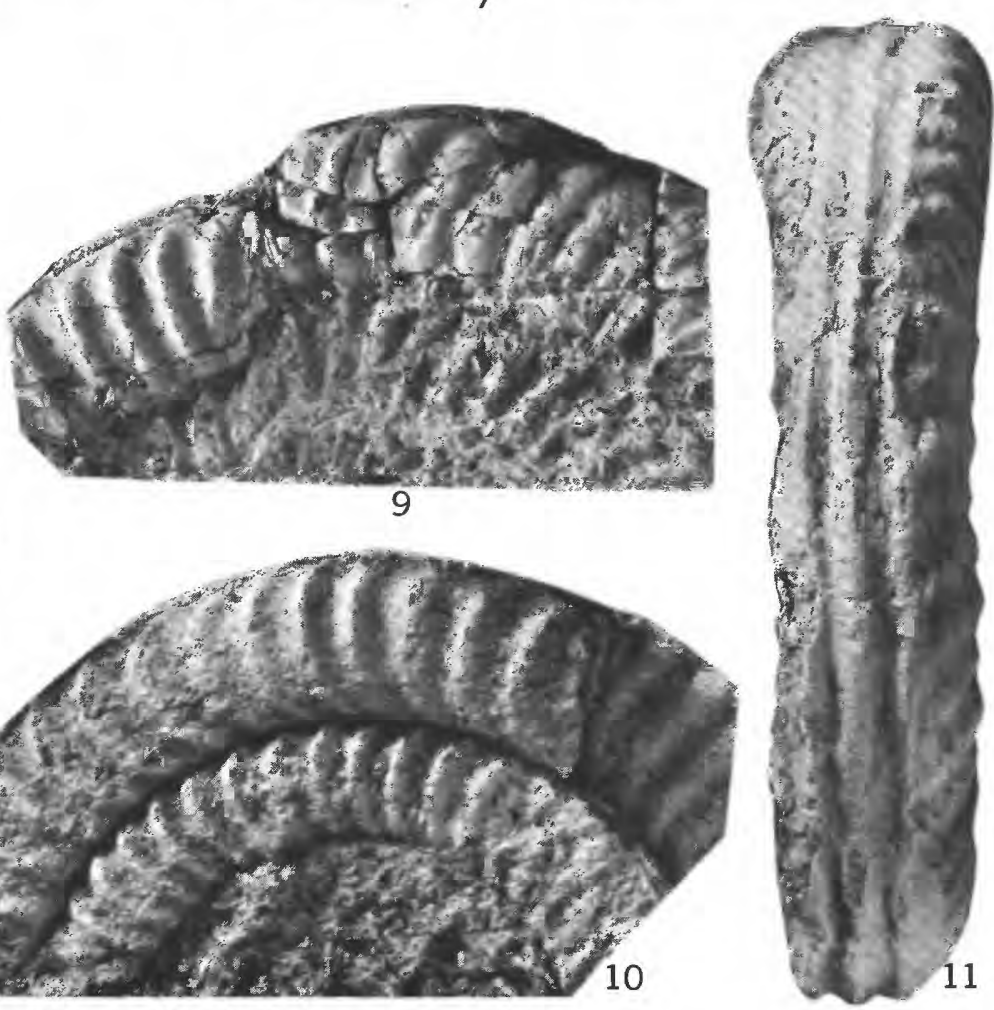


\section{PLATE 7}

[All figures are natural size]

Figures 1-3. Crucilobiceras cf. C. submuticum (Oppel) (p. 33).

1, 2. Specimen, USNM 248008, from USGS Mesozoic loc. 6706.

3. Specimen, USNM 248009, from USGS Mesozoic loc. 28661.

4, 5. Crucilobiceras cf. C. densinodulum Buckman (p. 34).

Specimen, USNM 248006, from USGS Mesozoic loc. 28661.

6-10, 12-15. Crucilobiceras cf. C. muticum (d' Orbigny) (p. 35).

Six specimens, USNM 248007, from USGS Mesozoic loc. 28661. Figures 9 and 10 represent ventral and lateral views of smallest specimen. Figures 12 and 13 represent same views of a fairly large specimen. Figures 14 and 15 represent same views of the largest specimen.

11. Fanninoceras kunae McLearn (p. 36).

Hypotype, USNM 248013, from USGS Mesozoic loc. 27586. 


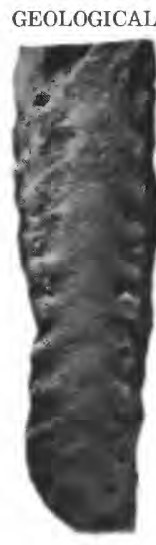

1
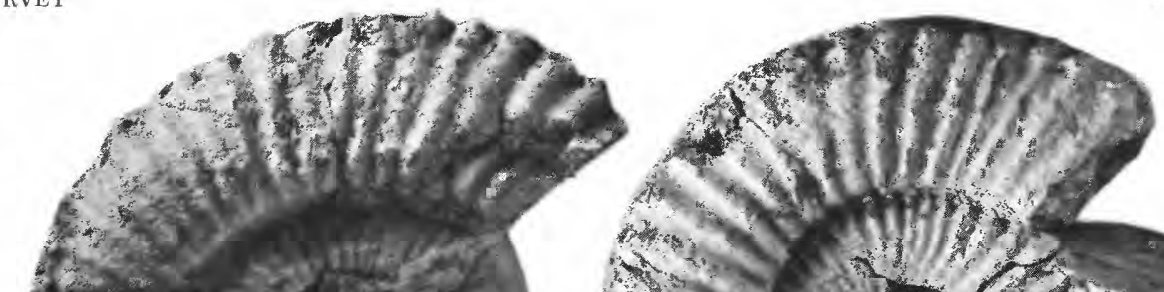

$i^{3}$ a

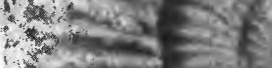

inction

not 3

66:

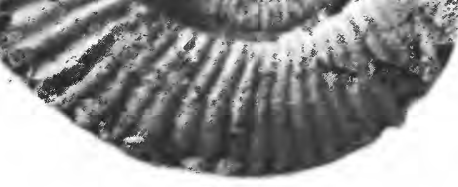

2

3 sin:

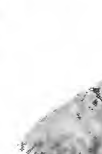

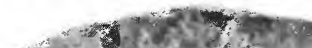
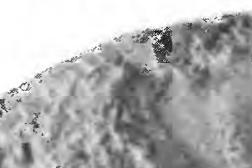

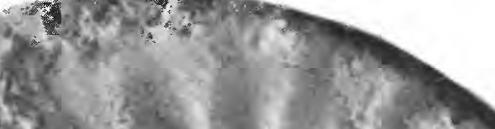

$+1$
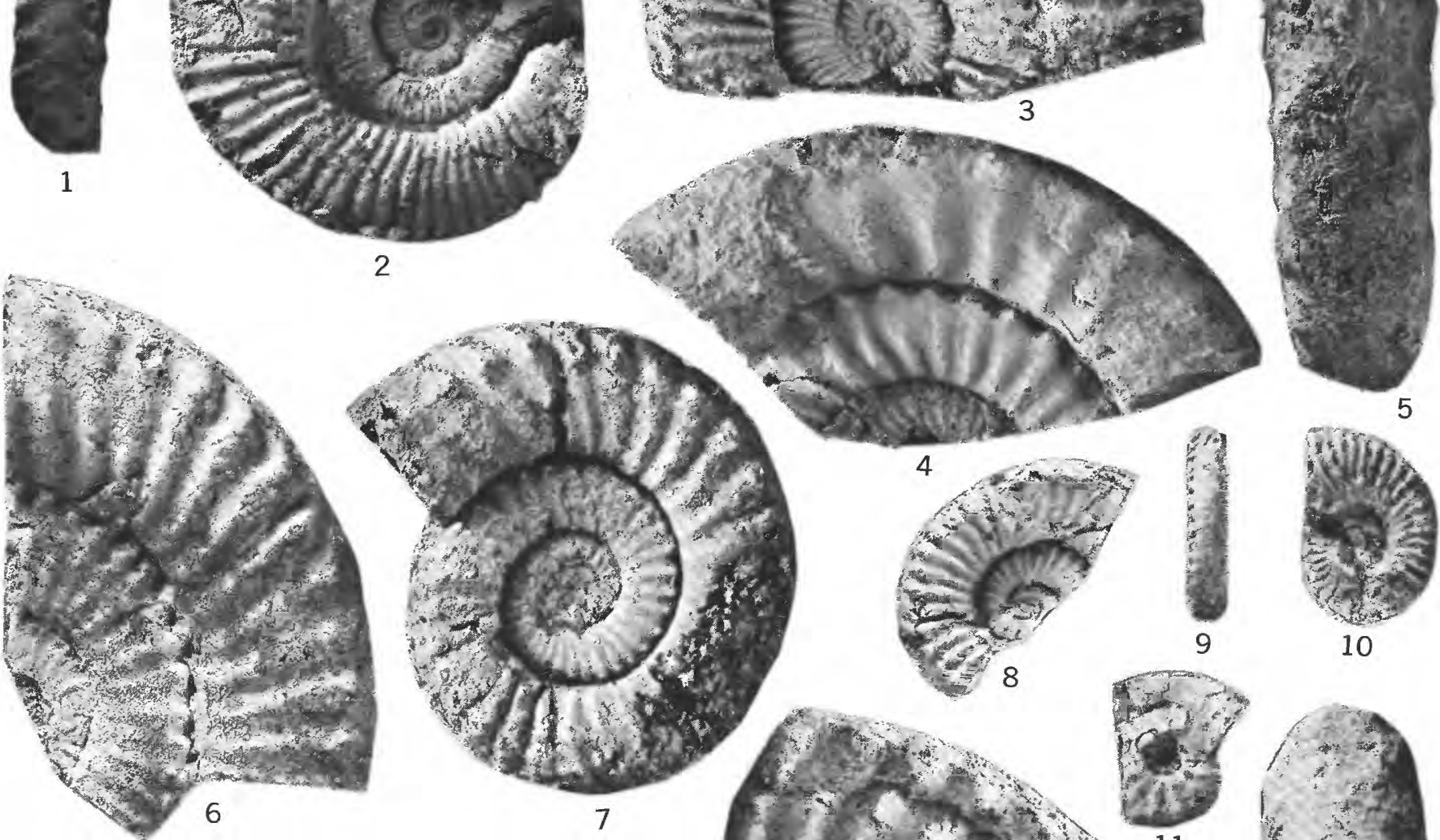

7

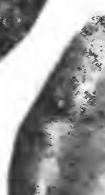

$\sqrt{20}=$

9

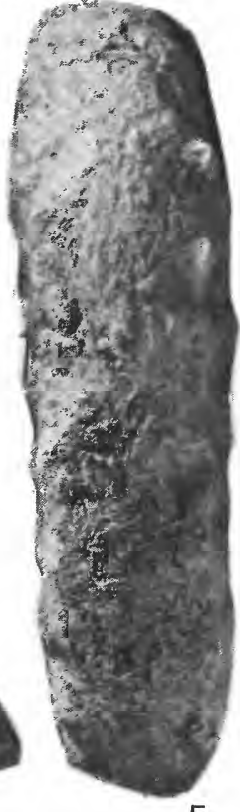

5
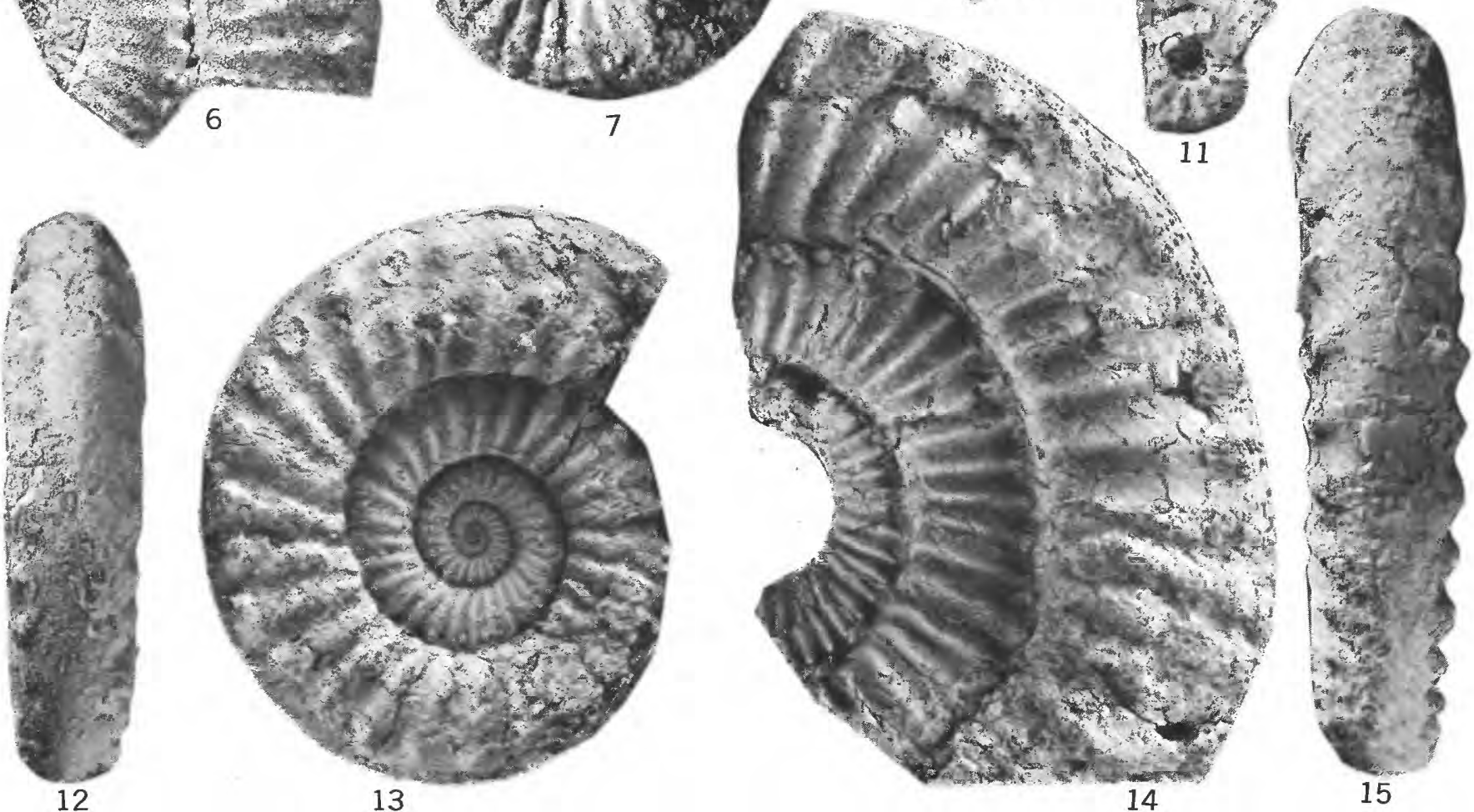

CRUCILOBICERAS AND FANNINOCERAS 


\section{PLATE 8}

[Figures natural size unless otherwise indicated]

Figures 1-9. Tropidoceras actaeon (d'Orbigny) (p. 37).

1-3. Hypotype, USNM 248021, from USGS Mesozoic loc. 28534.

4-6. Hypotype, USNM 248020, from USGS Mesozoic loc. 28534. Figure 4 is a rubber imprint of the external mold.

7. Hypotype, USNM 248022 , from USGS Mesozoic loc. 28534.

8, 9. Hypotype, USNM 248023, from USGS Mesozoic loc. 28534.

10-12, 15-17. Crucilobiceras cf. C. pacificum Frebold (p. 35).

One specimen, USNM 248010, from USGS Mesozoic loc. 28534. Figures $15-17(\times 2)$. Figure 12 is a rubber imprint of an external mold of the same specimen.

13. Uptonia? sp. A. (p. 36).

Specimen, USNM 248018, from USGS Mesozoic loc. 29773.

14, 18-23. Apoderoceras cf. A. subtriangulare (Young and Bird) (p. 35).

14,18-21. Specimen, USNM 248011, from USGS Mesozoic loc. 6697. Figures 18 and 19 represent the adapical part of the middle whorl shown on figure 21. Figure 14 does not include the cross section of the outermost whorl. Figure 20 is a rubber imprint of the external mold of the same specimen.

22, 23. Lateral and ventral views of a small specimen, USNM 248012, from USGS Mesozoic loc. M6171. 
GEOLOGICAL SURVEY
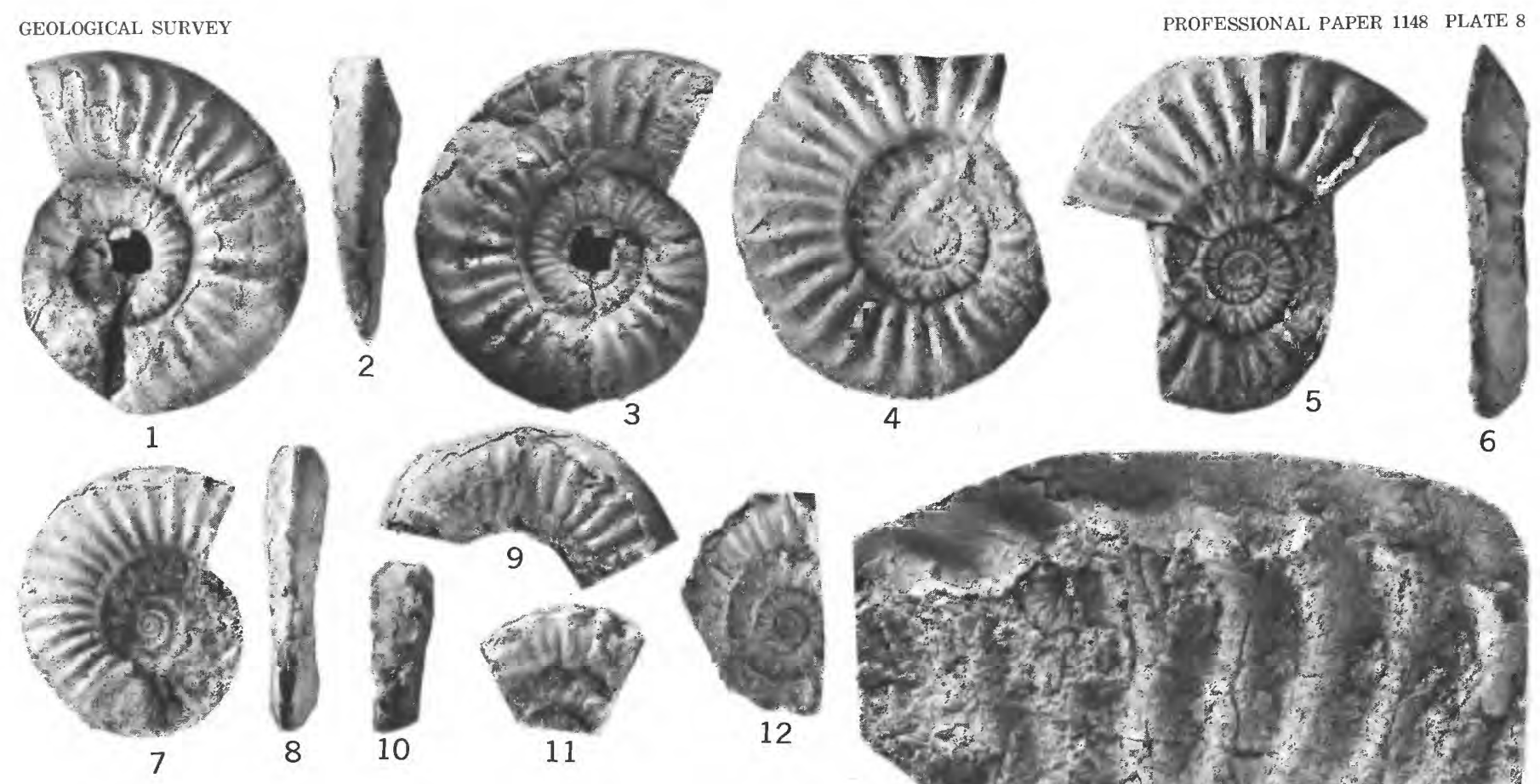

6

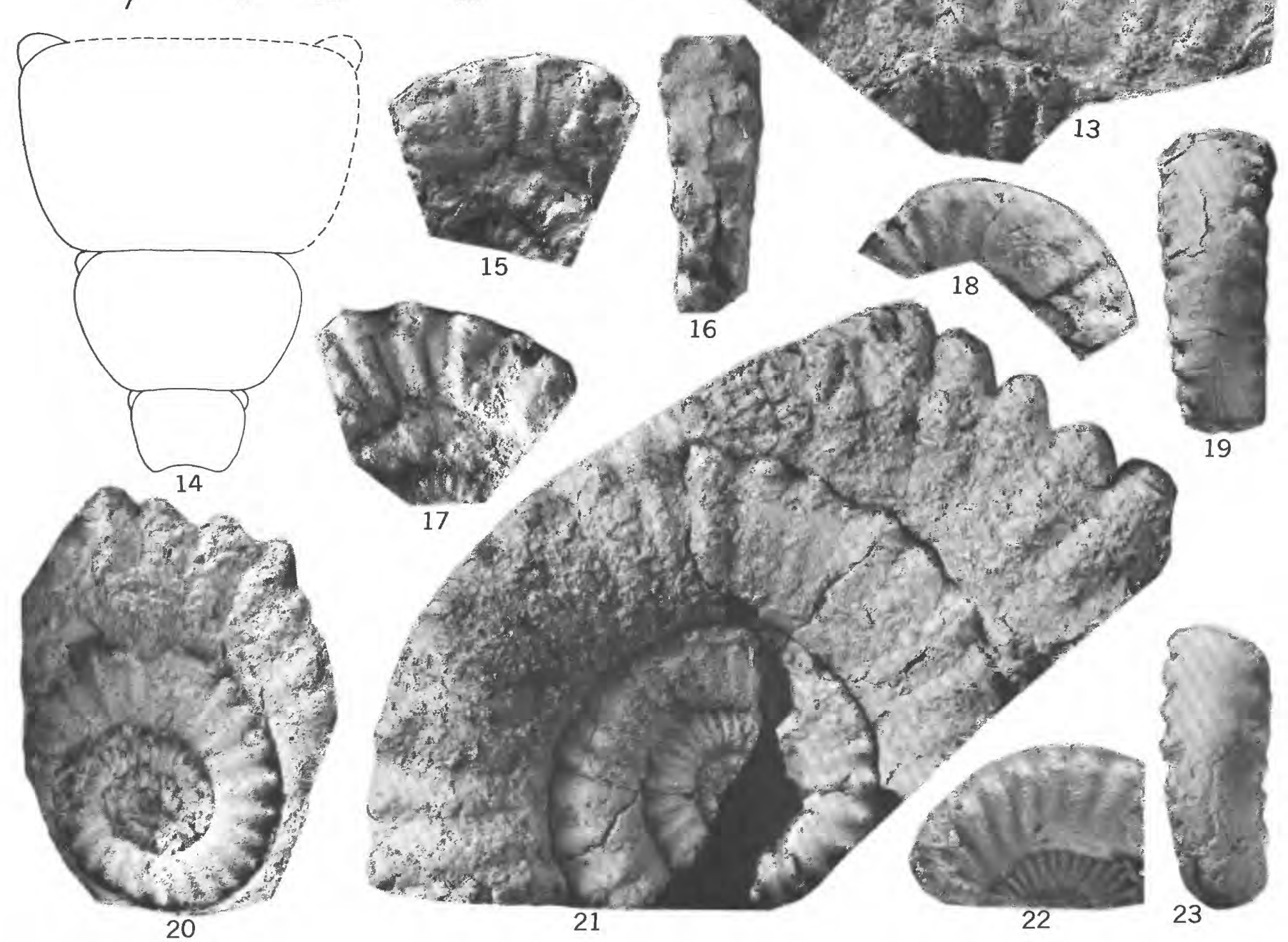

TROPIDOCERAS, CRUCILOBICERAS, UPTONIA?, AND APODEROCERAS 


\section{PLATE 9}

[Figures natural size unless otherwise indicated]

Figures 1-4, 8, 12-16. Uptonia cf. U. dayiceroides Mouterde (p. 36).

1-3. Specimens, USNM 248014, from USGS Mesozoic loc. 28675.

4, 8, 12-16. Specimens, USNM 248015, from USGS Mesozoic loc. 28671.

5-7. Uptonia sp. B (p. 37).

Specimen, USNM 248019, from USGS Mesozoic loc. 6697. Figures 5 and 7 are $(\times 2)$.

9-11. Uptonia? sp. (p. 36).

Specimen, USNM 248017, from USGS Mesozoic loc. 29775.

17. Uptonia cf. U. jamesoni (J. de C. Sowerby) (p. 36).

Laterally crushed specimen, USNM 248016, from USGS Mesozoic loc. 29774. 


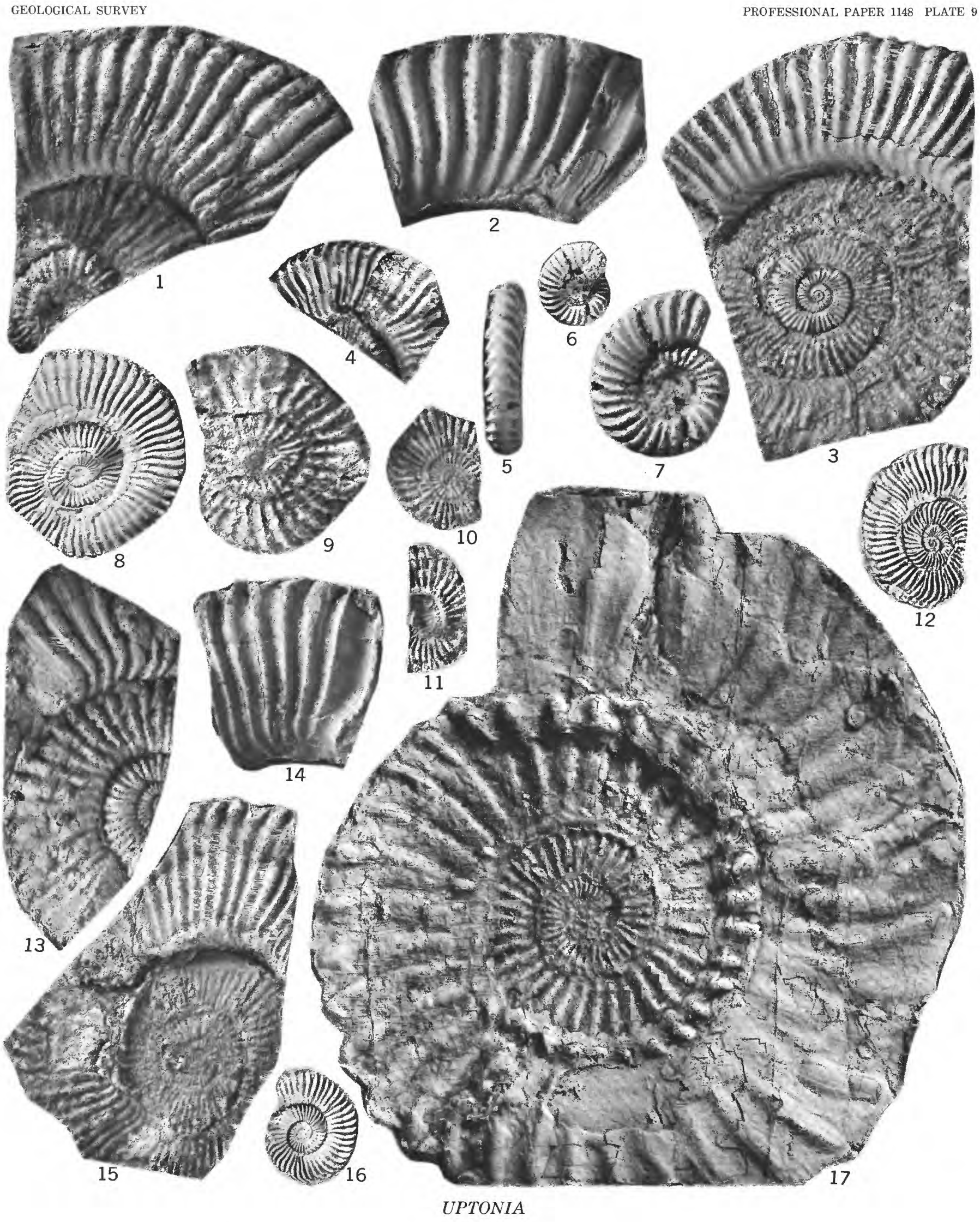




\section{PLATE 10}

[Figures natural size unless otherwise indicated]

Figures 1, 2, 6-15, 22. Arieticeras cf. A. domarense (Meneghini) (p. 39).

$1,2,8-10,15$. Specimens, USNM 248033, from USGS Mesozoic loc. 28688. Ventral and lateral views of one specimen are shown in figures 1 and 2. Same views of another specimen are shown in figures 9 and 10.

6. Specimen, USNM 248034, from USGS Mesozoic loc. 29450.

7. Specimen, USNM 248036, from USGS Mesozoic loc. 27586.

11-14. Two specimens, USNM 248035, from USGS Mesozoic loc. 24107. Ventral and lateral views of one specimen are shown in figures 11 and 12. Same views of another specimen are shown in figures 13 and 14.

22. Specimen, USNM 248037, from USGS Mesozoic loc. 28531.

3. Prodactylioceras italicum italicum (Fucini) (p. 38).

Hypotype, USNM 248030, from USGS Mesozoic loc. 28671.

4, 5. Prodactylioceras cf. P. italicum fucini Fischer (p. 38).

4. Specimen, USNM 248032, from USGS Mesozoic loc. 28673.

5. Specimen, USNM 248031, from USGS Mesozoic loc. 28671.

16-20. Arieticeras cf. A. algovianum (Oppel) (p. 40).

Specimens, USNM 248038, from USGS Mesozoic loc. 28688. Ventral and lateral views are shown in figures 16 and 17 , and also in figures 19 and 20.

21. Arieticeras sp. (p. 40).

Specimen, USNM 248039, from USGS Mesozoic loc. 28531.

23, 24. Amaltheus cf. A. stokesi (J. Sowerby) (p. 37).

23. Specimen, USNM 248029, from USGS Mesozoic loc. 30074.

24. Specimen, USNM 248028, from USGS Mesozoic loc. 25941.

25, 26. Amaltheus margaritatus (Montfort) (p. 37).

25. Hypotype, USNM 248025, from Simpson test well No. 1 at depth of $5,677 \mathrm{ft}(1,730 \mathrm{~m})$.

26. Hypotype, USNM 248024, from USGS Mesozoic loc. M2441.

27, 28. Amaltheus stokesi (J. Sowerby) (p. 37).

27. Hypotype, USNM 248026, from USGS Mesozoic loc. 29340.

28. Hypotype, USNM 248027, from USGS Mesozoic loc. 29165. 

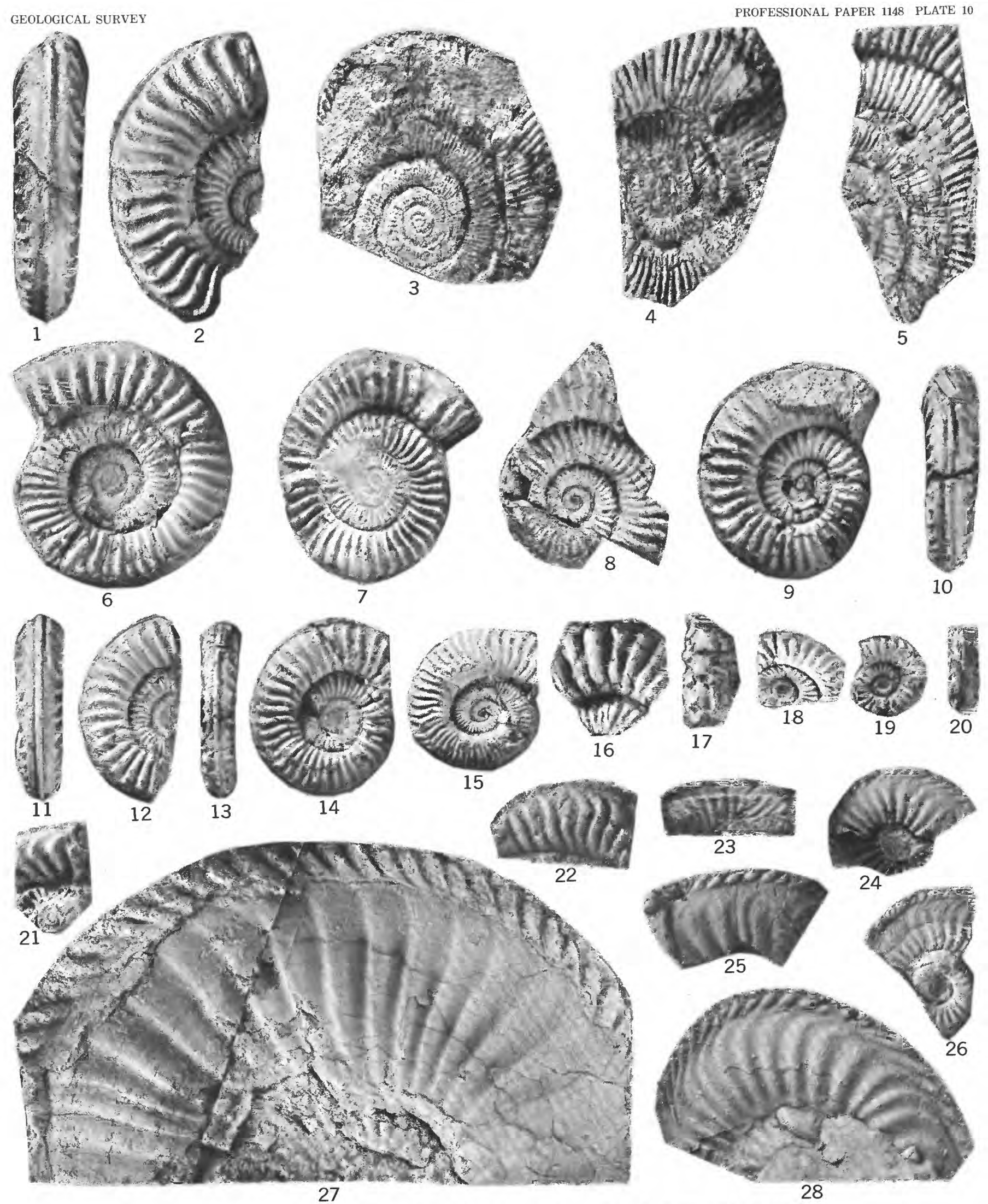

ARIETICERAS, PRODACTYLIOCERAS, ARIETICERAS, AND AMALTHEUS 


\section{PLATE 11}

[Figures natural size unless otherwise indicated]

Figure 1. Pseudolioceras sp. (p. 41).

Specimen, USNM 248052, from USGS Mesozoic loc. 19804.

2, 3, 8. Dactylioceras cf. D. commune (Sowerby) (p. 38).

Specimens, USNM 248047, from USGS Mesozoic loc. 24787.

4, 5, 9. Dactylioceras (Orthodactylites) kanense McLearn. (p. 39).

Hypotype, USNM 248048, from USGS Mesozoic loc. 29198. Figure 5 is a rubber imprint of an external mold.

6. Dactylioceras (Orthodactylites) cf. D. (O.) directum Buckman (p. 39).

Specimen, USNM 248049, from USGS Mesozoic loc. 29163.

7. Grammoceras sp. (p. 42).

Specimen, USNM 248053, from USGS Mesozoic loc. 25939.

10, 15, 16. Eleganticeras sp. juv. (p. 41).

Specimens $(\times 2)$, USNM 248050, from USGS Mesozoic loc. 29776. Figure 16 is a ventral view of figure 15.

11. Harpoceras cf. H. exaratum (Young and Bird) (p. 40).

Specimen, USNM 248044, from USGS Mesozoic loc. 29161.

12, 13. Leptaleoceras cf. L. pseudoradians (Reynes) (p. 40).

Specimen, USNM 248040, from USGS Mesozoic loc. 29450.

14. Protogrammoceras cf. P. argutum (Buckman) (p. 41).

Specimen, USNM 248046, from USGS Mesozoic loc. 25941.

17-23. Fontanelliceras cf. F. fontanellense (Gemmellaro) (p. 40).

7, 18, 23. Specimen, USNM 248043, from USGS Mesozoic loc. 29450.

19, 20. Specimen, USNM 248041, from USGS Mesozoic loc. 25941.

21, 22. Specimen, USNM 248042, from USGS Mesozoic loc. 27586. 


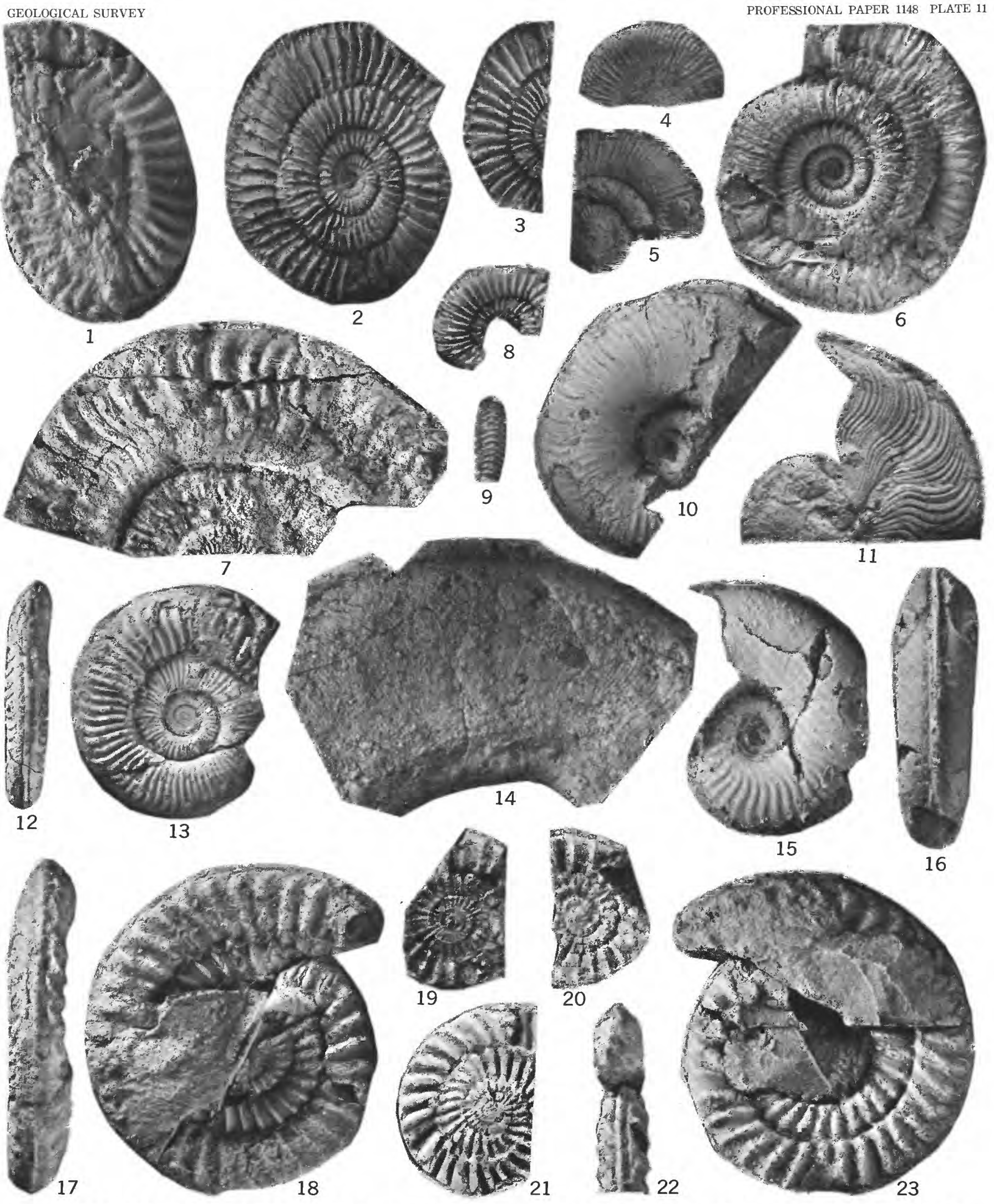

PSEUDOLIOCERAS, DACTYLIOCERAS, D. (ORTHODACTYLITES), GRAMMOCERAS, ELEGANTICERAS, HARPOCERAS, LEPTALEOCERAS, PROTOGRAMMOCERAS, AND FONTANELLICERAS 


\section{PLATE 12}

[All figures are natural size]

Figures 1, 2, 5. Haugia cf. H. variabilis (d'Orbigny) (p. 43).

Specimens, USNM 248059, from USGS Mesozoic loc. 25318.

3, 7, 9. Haugia cf. H. compressa Buckman (p. 43).

3, 9. Specimens, USNM 248060, from USGS Mesozoic loc. 24114.

7. Specimen, USNM 248061, from USGS Mesozoic loc. 19804.

6. Catacoeloceras? sp. juv. (p. 39).

Specimen, USNM 108758, from South Barrow No. 3 test well at depth of $2,063 \mathrm{ft}(629 \mathrm{~m})$.

4, 10, 15. Haugia cf. H. grandis Buckman (p. 42).

Lateral views of three specimens, USNM 248058, from USGS Mesozoic loc. 24114. Adoral part of figure 4 is an internal mold.

8. Brodieia cf. B. tenuicostata var. nodosa Jaworski (p. 42).

Specimen, USNM 248057, from USGS Mesozoic loc. 25940.

11, 12. Protogrammoceras ef. P. paltum Buckman (p. 41).

Specimen, USNM 248045, from USGS Mesozoic loc. 25941. Figure 12 is a rubber imprint of an external mold.

13. Pseudolioceras sp. (p. 41).

Specimen, USNM 248051, from USGS Mesozoic loc. 25317.

14, 16-19. Phymatoceras? sp. (p. 42).

14, 16. Specimen, USNM 248055, from USGS Mesozoic loc. 25317.

17, 19. Specimen, USNM 248054, from USGS Mesozoic loc. 25317.

18. Specimen, USNM 248056, from USGS Mesozoic loc. 25342. 


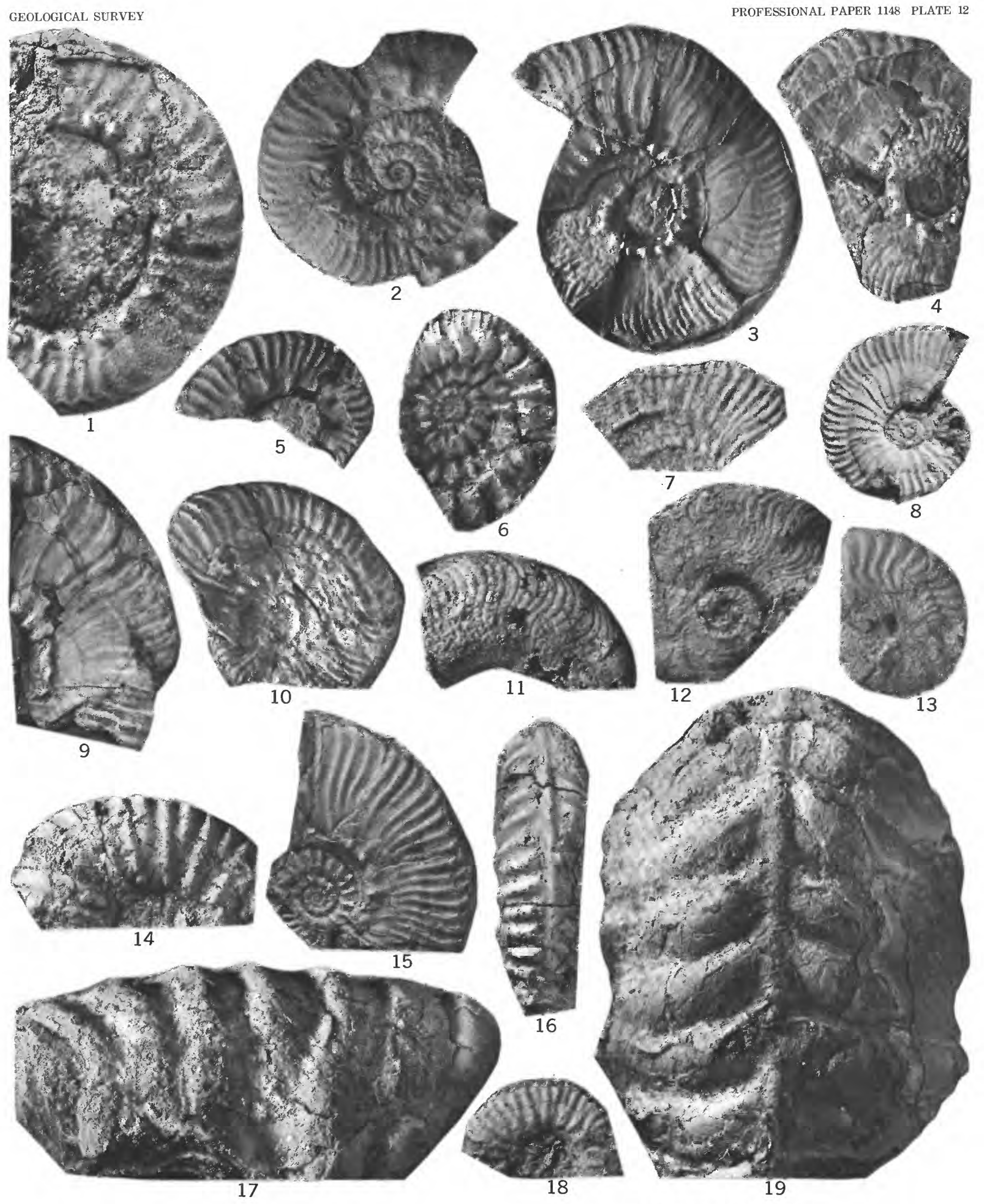

HAUGIA, CATACOELOCERAS, BRODIEIA, PROTOGRAMMOCERAS, PSEUDOLIOCERAS, AND PHYMATOCERAS? 